\title{
Exploring Mispricing in the Term Structure of CDS Spreads
}

\author{
Robert Jarrow, Haitao Li, Xiaoxia Ye, And May HU* \\ Forthcoming in Review of Finance
}

\begin{abstract}
Based on a reduced-form model of credit risk, we explore mispricing in the CDS spreads of North American companies and its economic content. Specifically, we develop a trading strategy using the model to trade out of sample market-neutral portfolios across the term structure of CDS contracts. Our empirical results show that the trading strategy exhibits abnormally large returns, confirming the existence and persistence of a mispricing. The aggregate returns of the trading strategy are positively related to the square of market-wide credit and liquidity risks, indicating that the mispricing is more pronounced when the market is more volatile. When implemented on the Markit data, the strategy shows significant economic value even after controlling for realistic transaction costs.
\end{abstract}

JEL Classification: G11, G12, G13, G14

Keywords: Credit default swaps, mispricing, statistical arbitrage, affine models, market-neutral strategy, hedge funds.

\footnotetext{
${ }^{*}$ Jarrow is with Cornell University and Kamakura Corporation; Li is with Cheung Kong Graduate School of Business; Ye is with University of Bradford and University of Liverpool; and Hu is with Deakin University. This paper was previously circulated under the title "Exploring Statistical Arbitrage Opportunities in the Term Structure of CDS Spreads" by the first three authors. We are grateful to the editor Amit Goyal and anonymous editor and referee for their suggestions and comments which substantially improved the paper. We also thank seminar participants at Xiamen University, Conference on Advances in the Analysis of Hedge Fund Strategies at Imperial College, National University of Singapore, Workshop on Financial Econometrics at University of Toronto; especially Fermin Aldabe, Peter Carr, Oliver Chen, Ying Chen, Hui-Ching Chuang, Jin-Chuan Duan, Peter Jackel, Hai Lin, Linlin Niu, Yu Ren, and Andrea Vedolin for helpful comments. The high performance computing resources provided by the National Supercomputer Centre in Sweden (NSC) at Linkoping University and Uppsala University are gratefully acknowledged. We are responsible for any remaining errors.
} 


\section{Introduction}

The credit derivatives markets have experienced tremendous growth in the last decade. According to the Bank for International Settlements (BIS), the notional value of outstanding credit derivatives peaked at the end of 2007 with $\$ 58$ trillion, then dropped sharply in 2008, and gradually stabilized at around \$26 trillion in recent years. The single-name credit default swaps (CDS) are believed to be the most liquid and popular product, as they account for more than two thirds of all outstanding credit derivatives. Though some exotic credit derivatives, such as subprime CDOs caused tremendous problems in the financial crisis, the vanilla CDS contracts play important economic roles. The newly proposed regulations, such as the establishment of a central clearing house for CDS, would help to reduce systemic risk and improve transparency in the CDS market. Therefore, the CDS contracts are likely to remain the preferred vehicle for investing, speculating, and managing single name credit risk. ${ }^{1}$

The rapid growth of the CDS market makes it possible to speculate on the relative pricing of the credit risk of a company across a wide range of maturities. Although five-year CDS have historically been the most liquid contracts, nowadays a complete credit curve (CDS spreads over different maturities) is available for many companies. As a result, it is possible to buy and sell protections on a given firm at different maturities. An interesting question to both academics and practitioners arises as to whether the credit risk of a firm is consistently priced across maturities. From an academic perspective, an important issue is whether existing credit risk models, either structural or reduced-form, can capture the rich term structure behaviors of credit spreads. From a practical perspective, one challenging issue is whether one can design trading strategies to exploit potential mispricings along the credit curve.

In two recent WSJ articles, Burne $(2015,2016)$ reports "Wall Street's biggest banks have agreed to a tentative settlement over allegations that they colluded to influence the market for credit derivatives. [...] The lawsuit brought by a group of investors accused the banks, the International Swaps and Derivatives Association and data provider Markit Group Ltd. of colluding to block competing providers, including exchanges, from entering the market for derivatives called credit-default swaps." The likely settlement

\footnotetext{
${ }^{1}$ See Oehmke and Zawadowski (2016) for a comprehensive discussion on the economic role of the CDS market. See Cui et al. (2013) for a study on the interaction between credit spreads, asset allocation, and liquidity risk.
} 
over these allegations casts doubt on the price discovery function and efficiency of the CDS market. Instead of directly studying the liquidity and competitiveness of the CDS market, our paper provides a test of the hypothesis that the CDS market is efficient given its current liquidity and competitiveness. As pointed out by Phillips and Smith (1980) and Hogan et al. (2004), the existence of abnormal returns after taking into consideration of proper trading costs rejects market efficiency. We provide strong evidence of the existence of mispricing in our empirical section below.

Using a reduced-form model of credit risk, we explore mispricing in the term structure of CDS spreads for a large number of North American companies. Specifically, we consider 500 firms with almost continuous daily observations of CDS spreads with maturities of one, two, three, five, seven, 10, 15, 20, and 30 years between January 2005 and October 2011. We estimate an affine model of credit risk for each company based on its term structure of CDS spreads and identify "mis-valued" CDS contracts relative to the model. Based on the estimated model parameters, we construct a portfolio of CDS contracts that are both delta- and gamma-neutral to potential changes in credit spreads. Then we long (short) the portfolio if it is under (over) valued relative to our model and unwind the portfolio a week later. We conduct both in-sample and out-of-sample analysis. In the in-sample analysis, we estimate model parameters, construct trading portfolios, and calculate trading profits using all of the data. In the out-of-sample analysis, we estimate model parameters using the first half of the sample, and we construct trading portfolios and calculate trading profits using the second half of the sample.

In both in-sample and out-of-sample exercises, the strategy generates significantly positive excess returns. These results, especially the out-of-sample tests, provide strong evidence of CDS mispricing. To explore the economic content of the mispricing, we average the out-of-sample returns across all firms and look at the relation between aggregate returns and various risk factors. We find that the aggregate returns are significantly and positively correlated with the square of market-wide credit and liquidity risks. This also means that the mispricing is more pronounced when the market is more volatile. Our robustness tests show the mispricing persists not only during the financial crisis period but in normal times. We do find evidence that the mispricing is more significant during the financial crisis period.

Although not our main focus, we also conduct a performance analysis accounting for realistic transaction costs. The purpose of the analysis is not to offer a complete and practical guide to the implemen- 
tation of a statistical arbitrage strategy, but merely to shed some light on its economic significance. This might be potentially useful: given the positive relation between aggregate returns and nonlinear risk factors, our trading strategy might provide quantitative CDS investors a means to obtain a highly profitable risk position. In this analysis, we find that our trading strategy is quite profitable: for half of the firms, the annualized Sharpe ratio is well above one in out-of-sample tests. The transaction costs included are as realistic as possible. For example, the included bid-ask spreads applying to the term structure of the CDS contracts are based on evidence documented in recent CDS empirical studies (see Biswas et al., 2014; Arakelyan and Serrano, 2012). We also find that the trading strategy has significant economic value in terms of the maximum performance fee due to Fleming et al. (2001) with respect to popular ETF funds, and the computed statistical arbitrage opportunities are statistically significant in terms of the formal test developed by Hogan et al. (2004).

Our paper contributes to three areas in the literature. Firstly, our study relates to the informational efficiency of CDS markets. There are only a few studies looking at the efficiency of the CDS market. Norden and Weber (2004), Zhang (2009), and Zhang and Zhang (2013) study the informational efficiency of the CDS market by analyzing the response of CDS to rating announcements, a variety of credit events, and earnings surprises, and they find evidence supportive of an efficient market. Recently, Du and Zhu (2015) study the design of CDS auctions and find that the current design leads to biased and inefficient prices. Although these studies have shed some light on the efficiency issue, and provide some interesting insights, they do not confront the core issue. As pointed out by Stulz (2010), the ultimate way to test the CDS market is to look at "the inefficiency brought about by limits of arbitrage — because otherwise arbitrageurs could exploit any mispricing of risk". We take the direct route by developing a trading strategy to test the pricing efficiency of the CDS market.

Second, our paper also contributes to the growing literature of persistent mispricing. For example, Mitchell et al. (2007) and Duffie (2010) discuss the role that slow-moving capital may play in allowing arbitrage opportunities to exist for extended periods of time. Fleckenstein et al. (2014) show the persistent mispricing driven by slow-moving capital exists in the TIPS-Treasury market. Meanwhile, Brunnermeier and Pedersen (2009) show that funding availability to intermediaries in financial markets is a potential explanation for deviations of security prices from the no-arbitrage conditions. Mayordomo et al. (2014) 
study the relative pricing between CDS and Asset Swap Packages (ASPs) and find persistent mispricing, however, they only consider the relative pricing between CDS and ASPs. Our study documents persistent mispricing in the CDS market that might be due to market manipulations (see, e.g., Allen et al., 2006; Aggarwal and $\mathrm{Wu}, 2006)$.

Our third contribution lies in the credit risk literature that has arisen in the past decade. There are many empirical studies on CDS involving the modeling of the entire credit curve given the increased availability of CDS spreads for a wide range of maturities. A few studies that are closely related to ours are Zhang (2008), Pan and Singleton (2008), and Chen et al. (2013), who estimate default risk models using the entire credit curve of CDS spreads. One contribution of our paper is that we are among the first to explore mispricing in the term structure of CDS spreads and its economic content.

The rest of this paper is organized as follows. In Section 2 we motivate our study. In Section 3 we develop a one-factor affine term structure model for CDS and discuss the econometric methods for estimating the model. Section 4 discusses the data and empirical results from the estimation. In Section 5 we discuss our trading strategy. We show the evidence of mispricing, explore and interpret its economic content in Section 6. Section 7 shows the net fee performance and economic significance of the trading strategy. Section 8 concludes. Appendices and online appendices contain technical details and supplementary results.

\section{Motivation}

The CDS market boomed after the Fed permitted banks to use CDSs to reduce capital reserves in 1996 (Levine, 2012). The growth of this market was exponentially fast from 2005 to 2007. The overall CDS market reached a notional value of \$58 trillion in 2007. Due to this explosive growth, market participants extensively use CDS for risk management and speculation (Gibson, 2007). This CDS usage might be partially due to the promotion of the CDS market by both the academic (e.g. Longstaff et al., 2005; Blanco et al., 2005) and regulatory communities (e.g. CDS implied default probabilities are being considered to replace credit ratings in U.S. financial regulations. Jarrow, 2012). This rapid rise of the 
CDS market (unintentionally) gave rise to a common impression that the market is liquid, competitive, ${ }^{2}$ and efficient.

This impression is debatable. van Deventer (2015) notes that $72.48 \%$ of the trading volume in single name CDS consists of only dealer to dealer trades. ${ }^{3}$ A problem with a dealer dominated market is that it is nearly costless for dealers to inflate gross trading volume by trading among themselves. However, this paper does not directly examine the liquidity of the CDS market since there are already extensive studies in the literature looking at this issue. Instead, we take the current liquidity of this market as given and focus on market efficiency. It is reported in recent WSJ articles Burne $(2015,2016)$ that 12 big banks and two industry groups ${ }^{4}$ have tentatively agreed to pay $\$ 1.87$ billion to settle allegations that they conspired to rig the CDS market. The accusations are that there was collusion among the banks and industry groups to block competing providers, e.g., exchanges from entering the CDS market. The simplest way of colluding is to manipulate the prices of CDS contracts. Indeed, the current CDS market with a majority of the players being institutional traders with large market power satisfies the classic conditions for market manipulation, see Jarrow (1992). Therefore, the pending settlement over these allegations suggests the possible existence of price manipulation in the CDS market. This brings the pricing efficiency of the market into question. As such, we explore the existence of mispricing in the CDS market and its economic content. It is evidenced in Allen et al. (2006) and Aggarwal and Wu (2006) that price manipulation is typically accompanied by increased volatilities. Therefore, in our empirical analysis we specifically look at the relation between mispricing and volatilities of various risks, e.g., credit and liquidity risks.

\footnotetext{
${ }^{2}$ By "competitive" we mean that all traders act as price takers, that is, traders have no control on prices (Jarrow and Larsson, 2012).

${ }^{3}$ van Deventer (2015)'s results are based on data provide by Depository Trust \& Clearing Corporation (DTCC) from July 16, 2010 through December 26, 2014. DTCC started providing trading volume data only after 2010. However, the trading volume was similar during our sample period (from 2005 to 2010).

${ }^{4}$ The 12 banks are Bank of America Corp., Barclays PLC, BNP Paribas SA, Citigroup Inc., Credit Suisse Group AG, Deutsche Bank AG, Goldman Sachs Group Inc., HSBC Holdings PLC, J.P. Morgan Chase \& Co., Morgan Stanley, Royal Bank of Scotland Group PLC and UBS Group AG. The two industry groups are the International Swaps and Derivatives Association and data provider Markit Group Ltd.
} 


\section{Model and Estimation Method}

\subsection{The Model}

In this section, we develop a one-factor affine model for the term structure of CDS spreads. We use only one factor to capture the dynamics of credit risk because our principal component analysis (PCA) shows that the first principal component captures $96 \%$ of the variations of CDS spreads. Our model is similar to that of Longstaff et al. (2005), Duffie and Singleton (1999), Duffie and Singleton (1997), Duffie et al. (2003), and Zhang (2008). We assume that credit spreads are independent of interest rates and thus avoid estimating a model for the risk-free term structure. As a robustness check, we run and obtain similar results using a two-factor affine model for the risk-free term structure, in which credit spreads are correlated with the two interest rate factors.

To investigate mispricing, we equate the "correct" price to the arbitrage-free price by assuming that the market is arbitrage-free and, hence, the existence of an equivalent martingale measure $Q$. Formally, we assume that the state variable, i.e. the default intensity $Z_{t}$, follows a square root process (CIR process) as

$$
d Z_{t}=\left(\alpha-\kappa Z_{t}\right) d t+\sigma \sqrt{Z_{t}} d w_{Z}^{Q}(t)
$$

where $w_{Z}^{Q}(t)$ is a standard Brownian motion under the equivalent martingale measure $Q$.

While we only need the dynamics of the state variable under the $Q$ measure for pricing purposes, we need its dynamics under the $P$ measure for econometric estimation. Given the extended affine specification for the market price of risk (Cheridito et al., 2007), we model the state variable's $P$ measure dynamics as

$$
d Z_{t}=\left(\alpha^{P}-\kappa^{P} Z_{t}\right) d t+\sigma \sqrt{Z_{t}} d w_{Z}^{P}(t) .
$$

The relation between the Wiener processes under the two measures is given by

$$
w_{Z}^{P}(t)=w_{Z}^{Q}(t)+\frac{\alpha-\alpha^{P}}{\sigma} \int_{0}^{t} \frac{1}{\sqrt{Z_{s}}} d s-\frac{\kappa-\kappa^{P}}{\sigma} \int_{0}^{t} \sqrt{Z_{s}} d s .
$$


To compute the CDS spread, we assume a constant recovery rate. Since both the buyer and the seller of credit protection in a CDS are exposed to counterparty risk, the quoted recovery rates might differ from the real recovery rates implicit in the CDS spreads. Therefore, unlike the common practice in the literature which fixes the recovery rate to a predetermined constant (see, e.g., Longstaff et al., 2005; Zhang, 2008), we estimate the value of the constant recovery rate along with the model parameters from the market prices of CDS spreads. Under the fractional recovery of face value (RFV) framework, which has been widely used for pricing CDS and is consistent with market practice, the recovery rate and the default intensity can be jointly identified in principle. To this end, we set recovery rate as $1-y=\exp \left(-\beta_{0}\right)$, where $\beta_{0}>0 .^{5}$

Then the CDS spread at time $t$ for protection between $t$ and $t+\tau$ satisfies

$$
S_{t}^{\tau}=\frac{\int_{t}^{t+\tau} P(t, u) \mathbb{E}_{2}(t, u)\left\{y-S_{t}^{\tau}\left(u-\frac{\llcorner 4 u\lrcorner}{4}\right)\right\} d u}{\frac{1}{4} \sum_{i=1}^{4 \tau} P\left(t, t+\frac{i}{4}\right) \mathbb{E}_{1}\left(t, t+\frac{i}{4}\right)}
$$

where $P(t, T)$ is the time $t$ price of a risk-free zero coupon bond that matures at time $T, S_{t}^{\tau}\left(u-\frac{\llcorner 4 u\lrcorner}{4}\right)$ reflects the accrued CDS premium from the previous payment date to the time of default, with $\llcorner 4 u\lrcorner$ denoting the largest integer smaller than $4 u$, and

$$
\begin{aligned}
& \mathbb{E}_{1}(t, u)=\mathbb{E}^{Q}\left[\exp \left(-\int_{t}^{u}\left(c_{0}+Z_{s}\right) d s\right) \mid \mathscr{F}_{t}\right] \\
& \mathbb{E}_{2}(t, u)=\mathbb{E}^{Q}\left[\exp \left(-\int_{t}^{u}\left(c_{0}+Z_{s}\right) d s\right)\left(c_{0}+Z_{s}\right) \mid \mathscr{F}_{t}\right] .
\end{aligned}
$$

Here, following Duffee (1999), the constant $c_{0}$ is added to improve the fit to the data. The detailed formulae for $\mathbb{E}_{1}(t, u)$ and $\mathbb{E}_{2}(t, u)$ are presented in Appendix A.

In practice, following Berndt et al. (2008) who use the midpoints between the quarterly payments,

\footnotetext{
${ }^{5}$ The positiveness of parameter $\beta_{0}$ ensures that $y \in(0,1)$.
} 
we discretize (4) as

$$
S_{t}^{\tau}=\frac{y \sum_{i=1}^{4 \tau} P\left(t, t+\frac{2 i-1}{8}\right) \mathbb{E}_{2}\left(t, t+\frac{2 i-1}{8}\right)}{\sum_{i=1}^{4 \tau} P\left(t, t+\frac{i}{4}\right) \mathbb{E}_{1}\left(t, t+\frac{i}{4}\right)+\frac{1}{8} \sum_{i=1}^{4 \tau} P\left(t, t+\frac{2 i-1}{8}\right) \mathbb{E}_{2}\left(t, t+\frac{2 i-1}{8}\right)} .
$$

In April 2009 ISDA implemented a number of CDS contract and convention changes known as the "CDS Big Bang". After the "CDS Big Bang", in general, CDS are quoted in upfront payments (or "upfronts"), which are the initial cash payments that compensate for the difference between a fixed coupon (100 or $500 \mathrm{bps}$ ) and the actual par spread. Given (7) and a fixed coupon $C$, the pricing formula of the upfronts at time $t$ for protection between $t$ and $t+\tau$ is

$$
U\left(Z_{t}, \tau\right)=\frac{y \sum_{i=1}^{4 \tau} P\left(t, t+\frac{2 i-1}{8}\right) \mathbb{E}_{2}\left(t, t+\frac{2 i-1}{8}\right)-C\left\{\sum_{i=1}^{4 \tau} P\left(t, t+\frac{i}{4}\right) \mathbb{E}_{1}\left(t, t+\frac{i}{4}\right)+\frac{1}{8} \sum_{i=1}^{4 \tau} P\left(t, t+\frac{2 i-1}{8}\right) \mathbb{E}_{2}\left(t, t+\frac{2 i-1}{8}\right)\right\}}{4} .
$$

The upfronts can be negative. If they are quoted as a negative then the protection buyer is paid the upfront fee by the protection seller; if the points are positive it's the other way around. Since we will work with the upfronts data in our analysis, we use expression (8).

\subsection{Model Estimation}

In this section, we discuss the econometric method for estimating our affine model using upfronts data. When implementing the model, we first need to back out zero yields from Treasury rates to compute the prices of the risk-free zero coupon bonds $P(t, T)$. Then, we use these zero coupon bond prices multiplied by the estimated discount factors $\mathbb{E}_{1}(t, T)$ to calculate the present value of the premium and the protection leg of the CDS contracts.

There are different econometric methods that one can use to estimate the affine model. Similar to Li et al. (2017), we use the unscented Kalman filter (UKF) in conjunction with QMLE to estimate the credit risk model. This is done because upfronts pricing formula is nonlinear in the state variable $Z_{t}$ (see Appendix B for details of the UKF). 


\subsubsection{State Space}

To use the UKF in empirical estimation, we re-cast our model in the framework of a state-space model. Although the transition density of the state variable in our model is not Gaussian, by applying the UKF with QMLE, we only need to consider the first two moments of the transition density. Therefore, we write down the transition equation as if the state variable is conditionally normally distributed, as long as the first two moments are intact. Duan and Simonato (1999) shows that this approximation is fairly efficient and accurate for estimating models with CIR type of state variables. Based on this approach, we provide the state-space representation of the defaultable term structure model below.

Let $\Delta t$ be the sampling interval in our study, which is a week. Then the transition equation for the default state variable $Z_{t}$ is given as

$$
\begin{aligned}
\mathbb{E}_{t-\Delta t}[Z(t)] & =\frac{\alpha^{P}}{\kappa^{P}}\left(1-\exp \left(-\kappa^{P} \Delta t\right)\right)+\exp \left(-\kappa^{P} \Delta t\right) Z(t-\Delta t) \\
\operatorname{Var}_{t-\Delta t}[Z(t)] & =\frac{\alpha^{P} \sigma^{2}}{2\left(\kappa^{P}\right)^{2}}\left(1-\exp \left(-\kappa^{P} \Delta t\right)\right)^{2}+\frac{\sigma^{2}\left(\exp \left(-\kappa^{P} \Delta t\right)-\exp \left(-2 \kappa^{P} \Delta t\right)\right)}{\kappa^{P}} Z(t-\Delta t)(.10)
\end{aligned}
$$

Let Upfronts ${ }_{t}^{\tau}$ be the actual upfronts quoted at $t$ for the protection between $t$ and $t+\tau$. Then the measurement equation becomes

$$
\mathrm{Upfronts}_{t}^{\tau}=U\left(Z_{t}, \tau\right)+\varepsilon_{t}^{\tau}
$$

where $\varepsilon_{t}^{\tau} \backsim$ i.i.d. $\mathscr{N}\left(0, v_{\tau}^{2}\right)$ and $\tau=1,2,3,5,7,10,15,20$, and 30 years. We assume the five-year upfront is priced without errors and set $v_{5}=0$.

\subsubsection{Likelihood Function}

We assume that the five-year upfronts are priced without error, and the measurement errors of other maturities are IID and normally distributed with zero mean. Then the transition density of

$$
\mathbf{S}(t)=\left[\begin{array}{llll}
\text { Upfronts }_{t}^{1} & \text { Upfronts }_{t}^{2} & \ldots & \text { Upfronts }_{t}^{30}
\end{array}\right]^{\top}
$$


given the information set $\mathscr{F}_{t-1}$ is a nine-dimensional normal distribution with mean $S_{t \mid t-1}$ and covariance matrix $P_{y_{t}}$, which are outputs from the UKF. Thus, the transition density of $\mathbf{S}(t)$ can be written as

$$
f_{t-1}(\mathbf{S}(t))=\left[(\sqrt{2 \pi})^{9} \sqrt{\left|P_{y_{t}}\right|}\right]^{-1} \exp \left[-\frac{1}{2}\left(\mathbf{S}(t)-S_{t \mid t-1}\right)^{\top} P_{y_{t}}^{-1}\left(\mathbf{S}(t)-S_{t \mid t-1}\right)\right]
$$

and the log-likelihood function is given by

$$
\ln \mathscr{L} \propto-\sum_{i=1}^{n} \ln \left|P_{y_{i}}\right|-\sum_{i=1}^{n}\left(\mathbf{S}(i)-S_{i \mid i-1}\right)^{\top} P_{y_{i}}^{-1}\left(\mathbf{S}(i)-S_{i \mid i-1}\right)
$$

where $n$ is the sample size.

In the estimation, we restrict $\alpha^{P}$ to be positive to ensure the existence of the CIR process (Feller, 1951). The $Q$ measure parameters are unconstrained. The positivity of the filtered CIR process is ensured by setting the joint likelihood of the entire time series to zero whenever the filtered CIR process is negative. Cheridito et al. (2007) adopt a similar method to impose boundary constraints on implied state variables in their estimation. Also, to ensure the parameter estimates, especially the recovery rate parameter, are not trapped in local optimums, we pre-estimate nine sets of parameters with $y$ fixed at 0.1 to 0.9 , then use the set of estimates giving the largest log likelihood value as the starting points in the full estimation.

\section{Empirical Analysis}

\subsection{Data}

The data used in our primary analysis here is from Markit. We also use GFI data for robustness tests which are presented in Appendix D. Based on the market makers' official books of record, live indicative quotes, and clearing submissions and results, Markit creates the daily composite spreads for each CDS contract. It also provides implied ratings over time for each firm. In the paper we use the implied ratings at the end of the sample period. We focus on US dollar denominated CDS contracts on all North America non-sovereign entities. We only use CDS on senior unsecured issues with modified 
restructuring (MR) clauses, as they are the most popular CDS contracts in the US market. To obtain accurate estimates of model parameters and to obtain enough observations for out-of-sample analysis, we require all firms included in our study to have a reasonably complete data coverage (over at least $75 \%$ daily coverage at each maturity) over the seven years (from Jan 2005 to Oct 2011). After applying this filter, we end up with 500 firms in our sample with daily CDS spreads for maturities of one, two, three, five, seven, 10, 15, 20, and 30 years. It is also reasonable to assume that these 500 firms are the most traded contracts, i.e., their CDS contracts are the most liquid, given they have the most complete data coverage. To speed up the estimation, we only use weekly (Friday) observations among these daily data. We believe these 500 firms represent the most traded single-name CDS contracts in the U.S. market. All the CDS spreads are converted to upfronts using the ISAD standard CDS converter, assuming the fixed coupon rate $C=100 \mathrm{bps}$ and a recovery rate of $40 \%$ (See ISDA, 2009). We bootstrap zero yields from the Constant Maturity Treasury (CMT) yields in release Fed H.15. The default-free discount factors used in our upfront pricing model are the prices of zero coupon bonds computed using these zero yields. ${ }^{6}$

Descriptive tables of the data are presented in the online appendix. Among the six ratings (AA, A, BBB, BB, B, and CCC), AA-, BBB-, and BB-rated firms account for $87 \%$ of the 500 firms. The table also contains the distribution of the firms among the ten different sectors, which include basic materials (BM), consumer goods (CG), consumer services (CS), financials (Fin), health care (HC), industrials (Ind), oil \& gas (OG), technology (Tec), telecommunication (Tel), and utilities (Uti). Fin have most firms, followed by Ind, CG, CS, OG, and Uti. Other industries have relatively fewer firms. The top three categories that have most firms are BBB-rated Ind (54), Fin (39), and OG (39).

Although we estimate our upfront pricing model using the upfronts converted from the CDS spreads, we present the data summary in terms of the CDS spreads for pedagogical reasons, as CDS spreads are closer to a traditional credit risk measure. For most ratings, we see an upward sloping credit curve, which is consistent with the notion that on average default risk is larger for longer maturities. For B- and CCC-

\footnotetext{
${ }^{6}$ We do not use LIBOR/Swap to construct benchmark zero yields as Dai and Singleton (2003), Duffie et al. (2003), and Zhang (2008) because our data covers the period after 2008 in which the LIBOR manipulation was occurring. See, e.g., Abrantes-Metz et al. (2012), McConnell (2013), Fouquau and Spieser (2015). This scandal resulted in the gradual elimination after 2008 of LIBOR as a benchmark rate due to a Wall Street Journal article by Mollenkamp and Whitehouse (2008). Therefore, it is improper to use LIBOR/Swap for CDS modeling especially during our sample period. We show in the online appendix the main results in Section 6 are robust to using Overnight Index Swaps (OIS) and Interest Rate Swaps (IRS) as discount rates.
} 
rated firms we observe a hump shaped curve peaking at the maturity of five-year. This is consistent with the notion that for speculative grade bonds, the default risk can be high in the near future but if the firm survives long enough, then the default risk actually goes down. The average credit curve for most sectors also slopes upward. One prominent exception is financial firms, whose CDS spread tends to decline with maturity. This is likely due to the fact that our sample covers the financial crisis period. During the crisis many financial firms were in trouble, therefore investors likely believe that these firms had higher credit risks in the short run than in the long run. In contrast, we find that the standard deviation of CDS spread generally declines with maturity. In general, lower rated firms have higher and more volatile CDS spreads than higher rated firms. One exception is that the A-rated firms actually have lower spreads than AA-rated firms. We believe this is probably because of too few A-rated firms (only five of them in total) in our sample.

\subsection{Estimation Results}

We estimate the credit risk model using the whole term structure of upfronts for each of the 500 firms. Table 1 presents the first, third quarters, median, and mean of variance ratios at different maturities. The variance ratio measures the percentage of variations of the upfronts explained by the model. The model explains the variations of most upfronts very well. At most maturities, the median variance ratios (both the full sample and first the half of the sample) are above $90 \%$. This suggests that our one-factor model does a reasonably good job in capturing the dynamics of the term structure of upfronts.

The estimation enables the model to fit the five-year CDS upfronts as well as possible (see Section 3.2.2) which are believed to be the most liquid and therefore the most informative about the credit risk of the underlying firm. ${ }^{7}$ The estimation results in Table 1 confirm that the one-factor model not only fits the five-year CDS upfronts perfectly (with the variance ratios at five-year being virtually 100\%) but that it is also able to fit the maturities around five years very well, for example, two, three, seven, 10, and 15 years. Apparently, the one-factor model fits less well at the very short and long ends of the maturity spectrum. Adding more latent factors would improve the fitting performance at both ends. However, our

\footnotetext{
${ }^{7}$ The fact that the five-year CDS are fitted perfectly does not mean that the trading strategy will not trade five-year CDS contracts. The five-year CDS contracts are traded as often as the other contracts because every contract is deployed in the trading strategy to form hedged portfolios. This will become clearer in Section 5.
} 
goal here is neither to perfectly fit the whole term structure nor to find the "best" model, but rather to explore the information content implicit in the pricing errors. Specifically, we are interested in determining whether deviations of market prices from our one-factor model indicate any profitable trading opportunities. Since our one-factor model in general captures over $90 \%$ of the variation in the term structure dynamics and fits the five-year maturity perfectly, we use our parsimonious one-factor specification to construct a trading strategy to explore the existence and economic content of any mispricings. It is also worth noting that if a "flawed" model (e.g. only one factor) generates significantly large excess returns, then the true mispricing in the market is even more pronounced. An improved model should generate even larger returns. ${ }^{8}$ Therefore our results should be regarded as the conservative documentation of potential mispricing.

Summaries of the parameter estimates are presented in Table 2. The $P$ measure parameters $\alpha^{P}$ and $\kappa^{P}$ imply a mean-reverting $Z_{t}$ for most of the firms. The median and average long-run mean $\left(\alpha^{P} / \kappa^{P}\right)$ of the processes are around $1.9 \%$ and $4.4 \%$. In contrast, the risk-neutral parameters $\alpha$ and $\kappa$ suggest an explosive $Z_{t}$ for most of the firms. This explains why in most cases we observe higher CDS spreads at the long maturities. This also implies that the expected rate of default intensity is lower under the physical measure than under the risk-neutral measure, indicating that investors require a premium for bearing exposure to varying default risk. This is consistent with similar findings in several papers (see, e.g., Duffee, 1999; Pan and Singleton, 2008; Jarrow et al., 2010; Filipović and Trolle, 2013). From the estimated recovery rate $1-y$, we find that the average is $62.7 \%$. In contrast, the average debt recovery rate measured by post-default trading prices of senior unsecured bonds as reported in Moody's special comment of "Corporate Default and Recovery Rates" over 2005 to 2011 is around 50.9\%. Therefore, our estimated recovery rate slightly overestimates Moody's recovery rate. Indeed, the Monte-Carlo simulation exercise in the online appendix shows that the model underestimates the loss by $12 \%$ when the true loss rate is $37 \%$. If we take this bias into consideration and adjust the recovery rate using the correction formula provided in the online appendix, we find that the corrected average recovery rate is $50.9 \%$ which is exactly Moody's estimate.

\footnotetext{
${ }^{8}$ This is supported in the robustness test in the appendix where a two-factor model is developed to fit another CDS dataset. The two-factor model significantly outperforms the one-factor model.
} 
Panel (a) of Table 3 presents the median of the adjusted long term default intensity mean, which is

given by $c_{0}+\frac{\alpha^{P}}{\kappa^{P}}$, in ratings and sectors. Except for the rating of $\mathrm{CCC}$, where the median adjusted long term mean is slightly lower than that of the B rating, we find that the lower the rating, the higher the adjusted long term mean. So the results confirm that default risks are larger for lower rated firms. From this panel, we also see that the telecommunication sector has the lowest adjusted long term mean, and that the consumer goods sector has the highest adjusted long term mean. The ranking among the sectors is different, however, during the first half of the sample period due to probably different macroeconomic environments. The ranking among the ratings is similar in the first half of the sample period. Only the A rating seems to have an abnormally large adjusted long term mean relative to other ratings during the first half sample. However, the A rating has only 5 firms, so the results might not be representative.

Panel (a) of Table 4 presents the median of the recovery rates across ratings and sectors. For ratings, the estimated recovery rates range from $41 \%$ (CCC) to $93 \%$ (A). Except for the A rating, we find a monotone relation between the median recovery rates and the ratings, i.e., the higher the rating the larger the median recovery. This is consistent with the intuition that the firms with higher ratings typically have larger recovery rates. The deviation of the median recovery rates among different sectors is smaller, as the range is from $46 \%$ (CG) to $68 \%$ (Ind). Again, the interpretation of these estimated recovery rates comes with a caveat that although the relative ranking among the recovery rates is accurate, the absolute level of the recovery rates is somewhat overestimated. According to the correction formula in the online appendix, the recovery rates of $41 \%$ (CCC), $93 \%$ (A), $46 \%$ (CG), and $68 \%$ (Ind) should be $33 \%$ (CCC), $76 \%$ (A), 37\% (CG), and 55\% (Ind), respectively.

Since our main focus, which is to develop a trading strategy, does not require an unbiased estimate of the recovery rate, and because our model fits the CDS upfront data reasonably well, we proceed using these parameter estimates without correction.

\section{Statistical Arbitrage Strategy}

In this section, we first briefly review the definitions of statistical arbitrage and explain how CDS contracts are traded. We then discuss the design and implementation of the strategy. 


\subsection{Definitions of Statistical Arbitrage}

Statistical arbitrage is typically referred to as trading strategies that rely on mathematical modeling techniques seeking profit opportunities from pricing inefficiencies (see, e.g., Whistler, 2004; Pole, 2007; Avellaneda and Lee, 2010). In the academic community, statistical arbitrage is defined more rigorously and often employed to develop statistical tools for testing market efficiency (Bondarenko, 2003; Hogan et al., 2004; Jarrow et al., 2012). For example, Hogan et al. (2004) define a statistical arbitrage to satisfy four conditions: a) it is a zero initial cost self-financing strategy, ${ }^{9}$ b) it has positive expected profits in the limit as time goes to infinity, c) the probability of a loss converges to zero, and d) if loss the probability is non-zero in finite time, a time-average variance converges to zero. Notice that the academic definition does not conflict with the practical one. The strategy we develop is consistent with both definitions. First, the practical implications of our strategy make it potentially useful for hedge funds and investment banks engaging in quantitative trading in the CDS market, while we admit that the technical details involved in actual trading is not our focus and depends on an idiosyncratic set of constraints faced by the arbitrageur (Fleckenstein et al., 2014); second, the previously developed statistical arbitrage test can be applied to the profits generated by our strategy to examine the extent of any mispricing in the CDS market.

\subsection{Trading CDS Contracts}

As mentioned before, after the "CDS Big Bang" the quoting convention for CDS contracts changed from quoting par spreads ${ }^{10}$ to quoting upfronts. With this quoting convention, the upfronts can be directly regarded as the price of the contract. The capital gain before transaction costs $\left(C G^{b}\right)$ of a trade is simply the notional amount (NA) times the positive or negative difference between the starting and ending upfronts of the trade depending on whether the trade is initially a long or a short position:

$$
C G_{t+\Delta t}=N A_{t} I_{t}\left(U \text { pront }_{t+\Delta t}-U \text { pront }_{t}\right)
$$

\footnotetext{
${ }^{9}$ Condition a) can be enforced by investing trading profits and borrowing trading losses and collateral (initial margin) at the risk-free rate (see Hogan et al., 2004, page 541). This is a part of the implementation of our trading strategy. Therefore, our trading strategy fits the formal definition while fulfilling a realistic collateral setup.

${ }^{10}$ This is the CDS spreads in the traditional sense. That is the par spread is the coupon a buyer is willing to pay on a quarterly basis over the life of the contract in exchange for protection against the default of the reference obligation, with no initial cash exchange except the cash exchanged as a collateral on the trade.
} 
where $\Delta t$ is the duration of the trade; $I_{t}$ is an indicator variable that equals 1 if the trade is initiated in a long position and -1 if it is initiated in a short position. The return of a trade is given by

$$
r_{t}=\frac{C G_{t}}{I M_{t-\Delta t}}
$$

where $I M_{t-\Delta t}$ is the initial margin posted at time $t-\Delta t$. In this section we abstract from transaction costs and focus primarily on the idea of the strategy itself while leaving the detailed discussion of transaction costs to Section 7.

\subsection{Design and Implementation}

The idea behind our trading strategy is to exploit the predictability in the pricing residuals produced by an arbitrage-free term structure model. Economically significant predictability in the pricing residuals in government bonds and LIBOR/Swap markets has been documented in Sercu and Wu (1997) and Bali et al. (2009). Here we develop a trading strategy similar to Bali et al. (2009) to explore mispricings in the CDS market. We rely on our estimated CDS pricing model to extract the state variable of the individual default risk (under the risk neutral measure) and produce pricing residuals. We construct market-neutral portfolios of CDS contracts that are immune to both the first and second order changes in the state variable. Then, we long (short) under (over) valued hedged portfolios. Here are the details of the strategy.

We consider the second-order expansion of the upfronts pricing function $U\left(Z_{t}, \tau\right)$ around the state variable $Z_{t}$ with the following first and second order derivatives $H_{1}\left(Z_{t}, \tau\right)=\left.\frac{\partial U(Z, \tau)}{\partial Z}\right|_{Z=Z_{t}}$ and $H_{2}\left(Z_{t}, \tau\right)=$ $\left.\frac{\partial^{2} U(Z, \tau)}{\partial Z^{2}}\right|_{Z=Z_{t}}$. The closed-form formulae of $H_{1}$ and $H_{2}$ are presented in Appendix C. Specifically, we have

$$
U\left(Z_{t+\Delta t}, \tau\right)=U\left(Z_{t}, \tau\right)+H_{1}\left(Z_{t}, \tau\right)\left(Z_{t+\Delta t}-Z_{t}\right)+\frac{1}{2} H_{2}\left(Z_{t}, \tau\right)\left(Z_{t+\Delta t}-Z_{t}\right)^{2}+O\left(\left(Z_{t+\Delta t}-Z_{t}\right)^{3}\right)
$$


We assume for $\Delta t=$ one week, ${ }^{11} U\left(\hat{Z}_{t+\Delta t}, \tau\right)$ can approximate $U\left(\hat{Z}_{t+\Delta t}, \tau-\Delta t\right)$ well, i.e.,

$$
U\left(Z_{t+\Delta t}, \tau-\Delta t\right) \approx U\left(Z_{t+\Delta t}, \tau\right)
$$

Then, by the above approximation and ignoring high order terms, (17) can be rewritten as

$$
U\left(Z_{t+\Delta t}, \tau-\Delta t\right) \approx U\left(Z_{t}, \tau\right)+H_{1}\left(Z_{t}, \tau\right)\left(Z_{t+\Delta t}-Z_{t}\right)+\frac{1}{2} H_{2}\left(Z_{t}, \tau\right)\left(Z_{t+\Delta t}-Z_{t}\right)^{2}
$$

Denote any maturity in $\left(\tau=1,2,3,5,7,10,15,20\right.$, and 30) by $\tau_{0}$, and the two adjacent maturities of $\tau_{0}$ by $\tau_{1}$ and $\tau_{2}$. To see how $\tau_{1}$ and $\tau_{2}$ are chosen, let us look at a few examples: if $\tau_{0}=1$, then $\tau_{1}=2$, and $\tau_{2}=3$; if $\tau_{0}=7$, then $\tau_{1}=5$, and $\tau_{2}=10$; and if $\tau_{0}=30$, then $\tau_{1}=15$, and $\tau_{2}=20$. Therefore, given $\Delta t$, at time $t$ for a unit notional amount of a CDS contract with maturity of $\tau_{0}$, we employ two other CDS contracts with maturities of $\tau_{1}$ and $\tau_{2}$ to form a hedged portfolio, whose value is immune to the variation of $Z_{t}$ up to the second order but subject to the variation of the pricing residuals.

To see this, let us recall our measurement equation (11) in Section 3.2.1 for $\tau_{0}$, $\tau_{1}$, and $\tau_{2}$ :

$$
\underbrace{\text { Upfronts }}_{3 \times 1} \vec{\tau}_{t}^{\vec{\tau}^{i}}=\underbrace{U\left(Z_{t}, \vec{\tau}^{i}\right)}_{3 \times 1}+\underbrace{\varepsilon_{t}^{\vec{\tau}^{i}}}_{3 \times 1}
$$

where $\vec{\tau}^{i}=\left[\tau_{0}, \tau_{1}, \tau_{2}\right]^{\top}$. Denote the portfolio weight by $\underbrace{M\left(Z_{t}, \vec{\tau}^{i}\right)}_{3 \times 1}$. We fix the first element in $M\left(Z_{t}, \vec{\tau}^{i}\right)$ at one for any $\overrightarrow{\tau^{i}}$. So the future value of the portfolio at time $t+\Delta t$ is given by

$$
\begin{aligned}
M\left(Z_{t}, \overrightarrow{\tau^{i}}\right)^{\top} \text { Upfronts }_{t+\Delta t}^{\vec{\tau}^{i}-\Delta t} & =M\left(Z_{t}, \vec{\tau}^{i}\right)^{\top} U\left(Z_{t+\Delta t}, \vec{\tau}^{i}-\Delta t\right)+M\left(Z_{t}, \vec{\tau}^{i}\right)^{\top} \varepsilon_{t+\Delta t}^{\vec{\tau}^{i}-\Delta t} \\
& \approx M\left(Z_{t}, \overrightarrow{\tau^{i}}\right)^{\top} U\left(Z_{t}, \overrightarrow{\tau^{i}}\right) \\
& +M\left(Z_{t}, \vec{\tau}^{i}\right)^{\top}\left[H_{1}\left(Z_{t}, \vec{\tau}^{i}\right)\left(Z_{t+\Delta t}-Z_{t}\right)+\frac{1}{2} H_{2}\left(Z_{t}, \overrightarrow{\tau^{i}}\right)\left(Z_{t+\Delta t}-Z_{t}\right)^{2}\right] \\
& +M\left(Z_{t}, \vec{\tau}^{i}\right)^{\top} \varepsilon_{t+\Delta t}^{\vec{\tau}^{i}}
\end{aligned}
$$

\footnotetext{
${ }^{11}$ The accuracy of the approximation is justified using simulation results. The numerical results are given in the online appendix.
} 
If the second and third elements of $M\left(Z_{t}, \overrightarrow{\tau^{i}}\right)$ are specified such that

$$
M\left(Z_{t}, \vec{\tau}^{i}\right)^{\top} H_{1}\left(Z_{t}, \vec{\tau}^{i}\right)=0, M\left(Z_{t}, \vec{\tau}^{i}\right)^{\top} H_{2}\left(Z_{t}, \vec{\tau}^{i}\right)=0
$$

i.e.,

$$
\begin{aligned}
M\left(Z_{t}, \vec{\tau}^{i}\right)^{\top} & =\left[1, m_{1}\left(Z_{t}, \overrightarrow{\tau^{i}}\right), m_{2}\left(Z_{t}, \overrightarrow{\tau^{i}}\right)\right] \\
m_{1}\left(Z_{t}, \overrightarrow{\tau^{i}}\right) & =\frac{H_{2}\left(Z_{t}, \tau_{0}\right) H_{1}\left(Z_{t}, \tau_{2}\right)-H_{1}\left(Z_{t}, \tau_{0}\right) H_{2}\left(Z_{t}, \tau_{2}\right)}{H_{1}\left(Z_{t}, \tau_{1}\right) H_{2}\left(Z_{t}, \tau_{2}\right)-H_{2}\left(Z_{t}, \tau_{1}\right) H_{1}\left(Z_{t}, \tau_{2}\right)} \\
m_{2}\left(Z_{t}, \vec{\tau}^{i}\right) & =\frac{H_{2}\left(Z_{t}, \tau_{0}\right) H_{1}\left(Z_{t}, \tau_{1}\right)-H_{1}\left(Z_{t}, \tau_{0}\right) H_{2}\left(Z_{t}, \tau_{1}\right)}{H_{1}\left(Z_{t}, \tau_{2}\right) H_{2}\left(Z_{t}, \tau_{1}\right)-H_{2}\left(Z_{t}, \tau_{2}\right) H_{1}\left(Z_{t}, \tau_{1}\right)}
\end{aligned}
$$

we have the hedged portfolio. That is

$$
\begin{aligned}
M\left(Z_{t}, \overrightarrow{\tau^{i}}\right)^{\top} \text { Upfronts }_{t+\Delta t} \vec{\tau}^{i}-\Delta t & \approx M\left(Z_{t}, \vec{\tau}^{i}\right)^{\top} U\left(Z_{t}, \tau\right)+M\left(Z_{t}, \vec{\tau}^{i}\right)^{\top} \varepsilon_{t+\Delta t}^{\vec{\tau}^{i}} \\
& =M\left(Z_{t}, \vec{\tau}^{i}\right)^{\top} \operatorname{Upfronts}_{t} \vec{\tau}^{i}+M\left(Z_{t}, \vec{\tau}^{i}\right)^{\top} \Delta \varepsilon_{t+\Delta t}^{\vec{\tau}^{i}}
\end{aligned}
$$

where $\Delta \varepsilon_{t+\Delta t}^{\overrightarrow{\tau^{i}}}=\varepsilon_{t+\Delta t}^{\overrightarrow{\tau^{i}}}-\varepsilon_{t}^{\overrightarrow{\tau^{i}}}$

For each $\overrightarrow{\tau^{i}}$, we can either long or short this hedged portfolio. The long (1) and short (-1) indicators are summarized in a $9 \times 1$ vector, $I_{t}$. Given $I_{t}$ and $M\left(Z_{t}, \overrightarrow{\tau^{i}}\right)$, the portfolio weight vector $W\left(Z_{t}, I, \vec{\tau}\right)$ for all the nine CDS contracts is given by

$$
\underbrace{W\left(Z_{t}, I_{t}, \vec{\tau}\right)}_{9 \times 1}=\underbrace{\left[I_{1, t} \vec{M}\left(Z_{t}, \overrightarrow{\tau^{1}}\right), I_{2, t} \vec{M}\left(Z_{t}, \overrightarrow{\tau^{2}}\right), \cdots, I_{9, t} \vec{M}\left(Z_{t}, \overrightarrow{\tau^{9}}\right)\right]}_{9 \times 9} \underbrace{\mathbb{1}}_{9 \times 1}
$$

where $\vec{\tau}=[1,2,3,5,7,10,15,20,30]^{\top}, I_{i, t}$ is the $i$ th element in $I_{t}, \vec{M}\left(Z_{t}, \vec{\tau}^{i}\right)$ is a $9 \times 1$ vector in which the elements corresponding to $\vec{\tau}^{i}$ in $\vec{\tau}$ are $M\left(Z_{t}, \vec{\tau}^{i}\right)$, and others are zero, ${ }^{12}$ and $\mathbb{1}$ is the vector of ones.

\footnotetext{
${ }^{12}$ For an illustration,

$$
\begin{aligned}
\vec{M}\left(Z_{t}, \overrightarrow{\tau^{1}}\right) & =\left[1, m_{1}\left(Z_{t}, \overrightarrow{\tau^{1}}\right), m_{2}\left(Z_{t}, \overrightarrow{\tau^{1}}\right), 0,0,0,0,0,0\right]^{\top} \\
\vec{M}\left(Z_{t}, \overrightarrow{\tau^{2}}\right) & =\left[m_{1}\left(Z_{t}, \vec{\tau}^{2}\right), 1, m_{2}\left(Z_{t}, \overrightarrow{\tau^{2}}\right), 0,0,0,0,0,0\right]^{\top} \\
\vec{M}\left(Z_{t}, \overrightarrow{\tau^{3}}\right) & =\left[0, m_{1}\left(Z_{t}, \overrightarrow{\tau^{3}}\right), 1, m_{2}\left(Z_{t}, \overrightarrow{\tau^{3}}\right), 0,0,0,0,0\right]^{\top} .
\end{aligned}
$$
}


Therefore the expected capital gain after transaction costs at time $t$ from holding the hedged portfolio of the whole term structure of CDS contracts for a period of $\Delta t$, given $I_{t}, W\left(Z_{t}, I_{t}, \vec{\tau}\right)$, and the forecast of $\Delta \varepsilon_{t+\Delta t}^{\overrightarrow{\tau^{i}}}, \mathbb{E}_{t}\left(\Delta \varepsilon_{t+\Delta t}^{\vec{\tau}}\right)$, is

$$
\begin{aligned}
\mathbb{E}_{t}\left(C G_{t+\Delta t}\right) & =W\left(Z_{t}, I_{t}, \vec{\tau}\right)^{\top} \mathbb{E}_{t}\left(\text { Upfronts }_{t+\Delta t}^{\vec{\tau}-\Delta t}-\text { Upfronts }_{t}^{\vec{\tau}}\right) \\
& \approx W\left(Z_{t}, I_{t}, \vec{\tau}\right)^{\top} \mathbb{E}_{t}\left(\Delta \varepsilon_{t+\Delta t}^{\vec{\tau}}\right) .
\end{aligned}
$$

We use the negative exponential moving average to forecast $\Delta \varepsilon_{t+\Delta t}^{\vec{\tau}}$, specifically,

$$
\mathbb{E}_{t}\left(\Delta \varepsilon_{t+\Delta t}^{\vec{\tau}}\right)=-\sum_{j=0}^{n}\left(1-\frac{2}{n+1}\right)^{j} \frac{2}{n+1} \Delta \varepsilon_{t-j \Delta t}^{\vec{\tau}}, n=4
$$

This is an exponential moving average of the past five weeks of $\Delta \varepsilon_{t}^{\vec{\tau}}$. The negative exponential moving average captures three characteristics of $\Delta \varepsilon_{t}^{\vec{\tau}}$ : a) the conditional mean is close to zero; b) the speed of mean reverting is large; and c) the recent lags are informative in forecasting next period's value.

Since we have nine maturities, at each point in time $t, I_{t}$ can be chosen from $\sum_{i=1}^{9} \frac{9 !}{i !(9-i) !}=511$ different vectors of -1 and 1 combinations. Denote the entire set of these 511 vectors by $\mathscr{I}$. At each point in time $t$, the portfolio weight $W\left(Z_{t}, I_{t}, \vec{\tau}\right)$ is chosen over $\mathscr{I}$ to maximize $\mathbb{E}_{t}\left(C G_{t+\Delta t}^{a}\right)$ :

$$
\tilde{W}_{t}=\left\{\begin{array}{cl}
W\left(Z_{t}, \tilde{I}_{t}, \vec{\tau}\right) & \text { if } W\left(Z_{t}, \tilde{I}_{t}, \vec{\tau}\right)^{\top} \mathbb{E}_{t}\left(\Delta \varepsilon_{t+\Delta t}^{\vec{\tau}}\right)>0 \\
0_{9 \times 1} & \text { if } W\left(Z_{t}, \tilde{I}_{t}, \vec{\tau}\right)^{\top} \mathbb{E}_{t}\left(\Delta \varepsilon_{t+\Delta t}^{\vec{\tau}}\right) \leqslant 0
\end{array},\right.
$$

where $^{13} \tilde{I}_{t}$ maximizes $(26)$, i.e.,

$$
\tilde{I}_{t}=\arg \max _{I_{t} \in \mathscr{I}}\left\{W\left(Z_{t}, I_{t}, \vec{\tau}\right)^{\top} \mathbb{E}_{t}\left(\Delta \varepsilon_{t+\Delta t}^{\vec{\tau}}\right)\right\}
$$

A real-life example of this weight calculation can be found in the online appendix.

\footnotetext{
${ }^{13}$ Notice that when $\tilde{W}_{t}=\mathbb{O}$ there is no trade at $t$, which does happen from time to time. Therefore even though the trading strategy monitors the dynamics of the CDS term structure weekly, it does not assume weekly trading in all CDS contracts. In other words, the inability to trade contracts weekly does not constrain the performance of our trading strategy in any way.
} 
The realized capital gain $C G_{t+\Delta t}$ is given by

$$
C G_{t+\Delta t}=s_{t}\left[\tilde{W}_{t}^{\top}\left(\operatorname{Upfronts}_{t+\Delta t}^{\vec{\tau}-\Delta t}-\text { Upfronts }_{t}^{\vec{\tau}}\right)\right]
$$

where $s_{t}$ is a scale factor and defined as

$$
s_{t}=\left\{\begin{array}{cc}
1 & \text { if } 0 \leqslant \tilde{W}_{t-\Delta t}^{\top} \mathbb{\mathbb { 1 }} \leqslant \bar{L} \text { or }-\underline{S} \leqslant \tilde{W}_{t-\Delta t}^{\top} \mathbb{\mathbb { 1 }} \leqslant 0 \\
\frac{\bar{L}}{\tilde{W}_{t-\Delta t}^{\top} \mathbb{1}} & \text { if } \tilde{W}_{t-\Delta t}^{\top} \mathbb{1}>\bar{L} \\
-\frac{S}{\tilde{W}_{t-\Delta t}^{\top} \mathbb{1}} & \text { if } \tilde{W}_{t-\Delta t}^{\top} \mathbb{1}<-\underline{S}
\end{array},\right.
$$

and $\bar{L}(\underline{S})$ is the cap on the net notional amount for a net long (short) position.

Once the model is estimated, $Z_{t}$ can be backed out by fitting the estimated model to the term structure of the observed Upfronts. Given the estimated model parameters and $Z_{t}, H_{1}\left(Z_{t}, \tau\right), H_{2}\left(Z_{t}, \tau\right), \varepsilon_{t}$ can be calculated for any $\tau$ and $t$, which then allow us to implement the above optimization procedure.

In the main test, we assume that we invest $\$ 1 \mathrm{M}$ as an initial deposit, i.e., $I M_{0}=\$ 1 \mathrm{M}$ which covers the initial margin for counterparty risk and collaterals for compensating the loss in case of an underlying default. With this $\$ 1 \mathrm{M}$, we are allowed to short with no more than the initial deposit, $\$ 1 \mathrm{M}$, and long with no more than three times the initial deposit, $3 \times \$ 1 \mathrm{M}=\$ 3 \mathrm{M}$. That is $\bar{L}=3 \times \$ 1 \mathrm{M}=\$ 3 \mathrm{M}$ and $\underline{S}=$ $\$ 1 \mathrm{M}$. During the sample period, we add cash to restore the account to $\$ 1 \mathrm{M}$ whenever the total money in the account is less than $\$ 1 \mathrm{M}$, which ensures no margin calls. We never withdraw cash from the account during the sample period, implying that if we are consistently making profit then the returns will be lower for a given capital gain.

\section{Evidence and the Economic Contents of Mispricings}

In this section, we first show evidence of the existence and persistence CDS market mispricings in terms of positive trading strategy returns. Then, we explore the economic contents of the mispricings by regressing the trading strategy returns onto various economic variables. 


\subsection{Trading Strategy Returns as a Mispricing Measure}

It is natural to use the returns generated from the trading strategy as a measure of mispricing in the sense of statistical arbitrage. If the strategy consistently generates positive returns, then the mispricing is persistent. We focus only on the out-of-sample performance of the strategy. We include the in-sample performance for comparison purposes in the next subsection and Section 7. Specifically, the model is estimated only using the first half of the full sample (January 2005 to June 2008); then based on the estimated model, the strategy is implemented on the second half of the full sample (July 2008 to October 2011). During each week, the weekly returns (16) are averaged cross-sectionally to form a time series of aggregate returns. We use these aggregate returns to analyze the overall mispricing in the CDS market. The larger the returns, the larger the mispricing.

It can be seen from the first panel in Fig. 1 that these returns are positive. The annualized return is $71.5 \%$ and annualized Sharpe ratio is 7.8. This is evidence supporting the existence of mispricings. The mispricing is more severe during the financial crisis period. From the second panel in Fig. 1, we can see that the aggregate returns are significantly smaller when the financial crisis period (December 2007 to June 2009) is excluded. ${ }^{14}$ This is also evident in the third panel of Fig. 1 where the returns cumulate faster before June 2009 than after. Indeed, without the financial crisis period, the annualized return reduces by almost half to $30.8 \%$. However, it is worth noting that although the annualized return reduces, the annualized Sharpe ratio more than doubles to 16.2, indicating the mispricings persist after the financial crisis with a lower volatility. These results are robust to the choice of the holding period and different data sources. Similar results are obtained using GFI CDS data. ${ }^{15}$ The robustness tests are presented in Appendix D and the online appendix.

\subsection{Risk Factors Explaining the Returns}

The previous analysis confirms the existence and persistence of mispricings. We also investigate whether the aggregate returns are correlated with certain systematic risks, especially the variance risk

\footnotetext{
${ }^{14}$ We use the NBER Business Cycles: http://www.nber.org/cycles.html to define the most recent financial crisis period.

${ }^{15}$ This is also true when changing the holding period to one month. See the online appendix of this paper for details.
} 
per the discussion in Section 2. To this end, we try to explain the aggregate returns using market-wide credit risk and liquidity risk. Specifically, we consider the following factors: ${ }^{16}$

CRDTSPRD: the weekly changes of the yield spread between Baa and Aaa bond indices. We use this series to represent the market-wide credit risk. Bali et al. (2009) and Hu et al. (2013) use the same factor.

NOISE: the weekly changes of the "Noise" measure of Hu et al. (2013). This is a market-wide liquidity measure which exploits the connection between the amount of arbitrage capital in the market and observed "noise" in U.S. Treasury bonds.

To control for sovereign default risk, business cycle risk, and systemic risk to the financial system, we also include the following variables in the regression as control variables: ${ }^{17}$

USCDS: the weekly changes of the five-year USA sovereign CDS spreads. We use this series as a proxy for market-based sovereign default risk.

NFCI: the weekly changes of National Financial Conditions Index (NFCI). This is a high frequency proxy for business cycle risk (see Brave and Butters, 2011, 2012).

MES: the weekly changes of average Marginal Expected Shortfall of 96 US banks/financial firm. We use this series as a proxy for systemic risk to the financial system.

To study the relation between the aggregate returns of our trading strategy and these factors, we regress the returns on these factors. To account for the variance of the main factors, we also include the squared terms of CRDTSPRD and NOISE on the right hand side (RHS). The regression results are reported in Table 5. ${ }^{18}$ From the results of regression (1), in which the RHS has only CRDTSPRD, NOISE, and the three control variables, we see the RHS has a fairly limited explanatory power, as

\footnotetext{
${ }^{16}$ CRDTSPRD are obtained from Federal Reserve Statistical Release; NOISE are generously provided by Jun Pan on her website.

${ }^{17}$ USCDS are from Credit Market Analysis (CMA); NFCI are from Federal Reserve Bank of Chicago: https://www . chicagofed.org/publications/nfci/index; MES are generously provided by the Volatility Laboratory of the NYU Stern Volatility Institute (https://vlab. stern.nyu .edu).

${ }^{18}$ The intercepts in the regressions should not be interpreted as alphas since the squared terms of the factors are not tradable. The purpose of running the regressions is to check if the aggregate returns are correlated with any of the factors.
} 
the adjusted $\mathrm{R}^{2}$ is less than $1 \%$ for the full sample returns; and in the second half sample, it is less than $5 \%$ for the in-sample returns and $12 \%$ for the out-of-sample returns. Moreover, the coefficients of CRDTSPRD and NOISE in regression (1) are never significant. Interestingly, the adjusted $\mathrm{R}^{2}$ 's increase dramatically after adding CRDTSPRD ${ }^{2}$ and NOISE $^{2}$ to the RHS: the adjusted $\mathrm{R}^{2}$ increases from $1 \%$ to $16 \%$ for the full sample returns, from $5 \%$ to $39 \%$ for the in-sample returns, and from $11 \%$ to $40 \%$ for the out-of-sample returns. Also, the coefficients of CRDTSPRD $^{2}$ and NOISE ${ }^{2}$ are all highly significant in regression (2), in which the RHS has CRDTSPRD, CRDTSPRD ${ }^{2}$, NOISE, NOISE ${ }^{2}$, and the three control variables in the three samples. The statistical significance of the coefficients and the adjusted $\mathrm{R}^{2}$ remain almost the same when moving from regression (2) to regression (3), where the RHS has only $\mathrm{CRDTSPRD}^{2}, \mathrm{NOISE}^{2}$, and the control variables. Looking at the control variables, none of them are significant in any of the regressions and samples. The same conclusion holds when the GFI CDS data are employed (see Appendix D).

These results indicate that the aggregate returns of the trading strategy are significantly related to the variance of market-wide credit and liquidity risk. The significantly positive coefficients of CRDTSPRD ${ }^{2}$ and NOISE ${ }^{2}$ show that when the credit market is volatile due to either credit or liquidity risk the mispricing is pronounced. Our analysis indicates that the mispricing stem from the strong predictive power of these nonlinear factors. Our trading strategy potentially provides an indirect tool that takes advantage of predictive power. This finding also echoes the price manipulation concern in Section 2 that motivates our study. See more discussion on this in the next subsection.

\subsection{Mispricing, Misspecification, or Risk Premium}

The fact that our trading strategy is based on pricing residuals of a reduced-form affine model might give an incorrect impression that the identified mispricing is due to model misspecification. However, we emphasize that this is not the case. We use the one-factor model to identify a common factor across different maturities and form portfolios that are immune to the changes in this factor. This is similar to Bali et al. (2009)'s idea of hedging away principal interest rate factors to explore predictability in residual factors. If the abnormal returns are due to model misspecification, then a better specified model should 
produce less prominent returns. However, we show in the online appendix that when based on a two factor affine model which fits the data better, our strategy produces even higher returns.

Our findings also relate to the well-known debate about whether abnormal returns are associated with mispricing or risk, see, e.g., Hirshleifer (2001) and Bloomfield and Michaely (2004). ${ }^{19}$ Although the two sides of this debate are not mutually exclusive,${ }^{20}$ they are traditionally considered to be competing alternatives. Two recent studies, Fleckenstein et al. (2014) and Mueller et al. (2017), on trading strategies exhibiting large excess returns distinctly classify themselves into these two sides.

Fleckenstein et al. (2014) attribute the excess returns they find to TIPS-Treasury mispricing as they show that the mispricing is a violation of the law of one price and therefore cannot be reconciled with an equilibrium model of asset pricing. They further provide direct evidence supportive of the slow-movingcapital explanation of arbitrage persistence. In contrast, Mueller et al. (2017) first develop a theoretical model showing that an increase in uncertainty regarding future interest rates in the United States results in higher excess returns for other currencies. Based on this model, they hypothesize that an increase in monetary policy uncertainty due to an upcoming FOMC announcement results in the depreciation of foreign currencies against the U.S. dollar, followed by an expected appreciation in the future. In their empirical analysis, they develop a trading strategy to verify the hypothesis and attribute the significantly larger excess returns on FOMC announcement days to monetary policy uncertainty premium.

Our study is consistent with Fleckenstein et al. (2014)'s mispricing story: we develop a market neutral trading strategy using portfolios of CDS contracts that consistently delivers positive excess returns. We provide further evidence that the realized excess returns are associated with the variances/volatilities of market-wide credit and liquidity factors and have small correlation with other systematic risk factors. Since increased volatility could be associated with price manipulation per Allen et al. (2006) and Aggarwal and Wu (2006), we conjecture that the identified mispricing could be attributed to potential manipulative behavior in the CDS market.

\footnotetext{
${ }^{19}$ We thank the anonymous editor for pointing out this topic.

${ }^{20}$ Mispricing per se can be a risk that carries premium, see, e.g., Brennan and Wang (2010) and Stambaugh and Yuan (2016). Fleckenstein et al. (2014) also admit that the difference between "mispricing" and what others call "risk premium" is a semantic one.
} 


\section{After Fee Performance and Economic Significance}

In this section, we present the performance and discuss economic significance of the trading strategy taking into account transaction costs. We show that our trading strategy is potentially useful for hedge funds and investment banks engaging in quantitative trading in the CDS market. However, we emphasize that this section is not to offer a complete and practical guide to the implementation of a statistical arbitrage strategy, which depends very much on the idiosyncratic set of constraints faced by any arbitrageur, and is beyond the scope of our academic study. Similar to Fleckenstein et al. (2014), our focal point is the identification of mispricings in the CDS market and the linkage between the mispricings and risk factors.

\subsection{After Fee Performance}

When there are transaction costs, the trading strategy needs to be slightly revised. The revised version and the transaction cost assumptions are presented in the online appendix. Given the assumptions, while the Markit data still carries economically significant profitability, the GFI data, which has much lower weekly volatilities ${ }^{21}$ fails to deliver decent performance. The analysis in this section is therefore only based on Markit data.

We provide in-sample and out-of-sample analysis on the profitability of our trading strategy. We divide our data into two parts, with the first part covering January 2005 to June 2008 and the second part covering July 2008 to October 2011. In the in-sample analysis, we estimate model parameters and construct trading portfolios using data that cover the entire sample period. In the out-of-sample analysis, we estimate the model parameters using the first half of the data, implement the strategy and compute the in-sample performance using the second half of the data.

We consider three important performance measures: the annualized total excess return (XR), the annualized Sharpe ratio (SR), and the maximum draw-down (MDD). XR measures how a trading strategy

\footnotetext{
${ }^{21} \mathrm{We}$ are not in the position to discuss why the GFI data have much lower volatilities than the Markit data. The lower volatilities, nevertheless, explains why the GFI data fails to deliver a decent after fee performance: if the average weekly changes of the CDS upfronts are smaller than the assumed bid-ask spreads, then any potential profits will be shadowed by the cost. Indeed, the average weekly changes in the Markit data are of two to three times the size of the assumed bid-ask spreads, while that of the GFI data are similar to if not much smaller than the assumed bid-ask spreads.
} 
outperforms a benchmark investment in terms of returns, but does not adjust for the trading risks. SR measures the risk (standard deviation of excess returns) adjusted excess returns of a trading strategy. MDD measures the downside risk of a trading strategy. They are defined as:

$$
\begin{aligned}
\mathrm{XR} & =\left\{\prod_{i=1}^{T}\left(1+r_{i \Delta t}^{a}\right)\right\}^{\frac{52}{T}}-1-r_{0, \text { a, risk-free }}^{\frac{T}{52}}, \\
\mathrm{SR} & =\frac{\sqrt{52}\left\langle r_{i \Delta t}^{a}-r_{(i-1) \Delta t, \mathrm{w}, \text { risk-free }}^{\frac{1}{52}}\right\rangle_{i=1}^{T}}{\operatorname{std}\left(r_{i \Delta t}^{a}-r_{(i-1) \Delta t, \mathrm{w}, \text { risk-free }}^{\frac{1}{52}}\right)_{i=1}^{T}} \\
\mathrm{MDD} & =\max _{i=1: T}\left\{\frac{\max _{j=1: i}\left(\sum_{k=1}^{j} C G_{k \Delta t}^{a}\right)-\sum_{k=1}^{i} C G_{k \Delta t}^{a}}{\max _{j=1: i}\left(\sum_{k=1}^{j} C G_{k \Delta t}^{a}\right)+I M_{0}}\right\},
\end{aligned}
$$

where $T$ is the total number of weeks during the period; $r_{t}^{a}$ is the after transaction cost weekly return at time $t$ and given in the online appendix; $r_{t, \text { a, risk-free }}^{\tau}$ and $r_{t, \mathrm{w}}^{\tau}$, risk-free are respectively the risk-free annual and weekly return for the period of $\tau$ at $t ;\langle\cdot\rangle_{i=1}^{T}$ and $\operatorname{std}(\cdot)_{i=1}^{T}$ denote respectively the average and standard deviations of the variable ${ }_{i}$ for $i=1, \cdots, T ; C G_{t}^{a}$ is the after transaction cost weekly capital gain at time $t$ and given in the online appendix.

A general summary of these measures is reported in Table 6. Among the 500 firms the median and mean of the full sample XR are $15 \%$ and $17 \%$, respectively. As for the full sample SR, the median and mean are 1.29 and 1.27 , respectively. The full sample MDD is on average only $11 \%$. In general, the full sample results show that the trading strategy makes profits in-sample from 2005 to 2011. These results carry over to the out-of-sample period, as is evident from the left panels of Table 6. During the second half of the sample (Jun 2008 to Oct 2011), out-of-sample performance is comparable to the in-sample performance with only small differences in the three measures: the median XR is $26 \%$ out-of-sample and $27 \%$ in-sample, the median SR is 1.5 out-of-sample and 1.7 in-sample, and the median MDD is $9 \%$ out-of-sample and $7 \%$ in-sample.

We also break down the results of the these three measures into different ratings and sectors, and present them in Tables 7 to 9. Since it is more meaningful to focus on the out-of-sample performance, we mostly discuss the out-of-sample results hereafter. In Table 7, we see a monotone increase (except 
for $\mathrm{CCC}$ ) in the median out-of-sample XR's from $18 \%$ for AA to $45 \%$ for B. As shown in Table 8 , we observe the same pattern for the median out-of-sample SR's, they range from 1.2 for AA to 1.5 for CCC. Firms with lower ratings normally have larger probabilities of default than those with higher ratings. The same pattern also exists in the median out-of-sample MDD's across ratings. They are all quite low, ranging from $7 \%$ for AA to $11 \%$ for CCC. This shows that the trading strategy has very little downside risk. Since our XR and SR do not adjust for credit risk, these monotone results indicate that profits from trading lowly rated firms carry a risk premium.

When we look at the performance across different sectors, we find that our strategy performs equally well in all sectors. For the median out-of-sample XR, Tel has the lowest XR of $19 \%$ and CG has the largest XR of $43 \%$. For the median out-of-sample SR, Tec has the lowest SR of 1.1 and OG has the largest SR of 1.7. For the median out-of-sample MDD, OG has the lowest MDD of $8 \%$ and BM has the largest MDD of $15 \%$. To save space, more results on the performance are contained in the online appendix.

\subsection{The Economic Significance of the Trading Strategy}

To conduct an economic analysis of the trading strategy, we look at the returns and capital gains on an aggregate level. Specifically, we average (add up) the cross-sectional after fee returns (after fee capital gains) from trading the individual term structure of CDS contracts over the sample period. Therefore, the time series of the aggregate returns $r_{\mathrm{Arb}}$ (capital gain $C G$ ) can be viewed as the returns (profits) of a fund investing in the CDS market by applying the trading strategy to the 500 single-names. We measure the economic significance of the trading strategy using break-even performance fees with respect to ETF funds and formally test the extent of mispricing using statistical measures.

To measure the economic significance of the trading strategy, we compare the performance of the dynamic trading strategy, to that of a static strategy that has the same target volatility. Following Fleming et al. (2001), we estimate the economic significance using a utility-based measure, which measures the performance fee $\Delta$ that an investor would be willing to pay to switch from a static strategy to a dynamic 
one. ${ }^{22}$ Specifically, we find the value of $\Delta$ that solves

$$
\sum_{t=0}^{T-1}\left(R_{\mathrm{arb}, t+1}-\Delta\right)-\frac{\gamma}{2(1+\gamma)}\left(R_{\mathrm{arb}, t+1}-\Delta\right)^{2}=\sum_{t=0}^{T-1} R_{\mathrm{sta}, t+1}-\frac{\gamma}{2(1+\gamma)} R_{\mathrm{sta}, t+1}^{2}
$$

where $\gamma$ represents the value of the investor's relative risk aversion; $R_{\mathrm{arb}, t}$ and $R_{\mathrm{sta}, t}$ are adjusted total returns, which have the same target volatility $\sigma_{\text {trgt }}$ from investing in the dynamic trading strategy and the static strategy, respectively. Specifically,

$$
\begin{aligned}
& R_{\mathrm{arb}, t}=1+\left(1-\frac{\sigma_{\mathrm{trgt}}}{\hat{\sigma}_{\mathrm{arb}, t}}\right) r_{f}+\frac{\sigma_{\mathrm{trgt}}}{\hat{\sigma}_{\mathrm{arb}, t}} r_{\mathrm{arb}, t}, \\
& R_{\mathrm{sta}, t}=1+\left(1-\frac{\sigma_{\mathrm{trgt}}}{\hat{\sigma}_{\mathrm{sta}}}\right) r_{f}+\frac{\sigma_{\mathrm{trgt}}}{\hat{\sigma}_{\mathrm{sta}}} r_{\mathrm{sta}, t},
\end{aligned}
$$

where $r_{\mathrm{sta}, t}$ is the gross return from a static strategy, $\hat{\sigma}_{\mathrm{arb}, t}$ is the ex post estimate of conditional volatility of $r_{\text {arb }, t}$, and $\hat{\sigma}_{\text {sta }}$ is the sample standard deviation of $r_{\text {sta, } t} \cdot{ }^{23}$

Here we consider three popular ETF funds in different markets (featuring the bond, equity, and volatility risks) as benchmarks: a) iShares iBoxx Investment Grade Corporate Bond ETF (LQD), which seeks to track the investment results of an index composed of U.S. dollar-denominated, investment grade corporate bonds and therefore represents the performance of the U.S. corporate bond market; b) SPDR S\&P 500 ETF (SPY), which seeks to track the investment results of the S\&P 500 Index and therefore represents the overall performance of the U.S. equity market; and c) iPath S\&P 500 VIX ST Futures ETN (VXX), which is designed to provide exposure to the S\&P 500 VIX Short-Term Futures Index Total Return and therefore represents the returns of bearing the overall volatility risk. These are the most heavily traded ETFs in the market.

We obtain the historical data for these ETF funds from Yahoo Finance, and calculate the weekly gross returns (before expenses) during the period matching our out-of-sample period. Based on our outof-sample returns and those of the ETF funds, we estimate the performance fees according to (34) for $\gamma=$ 1 and 10 with $\sigma_{\text {trgt }}=3 \%$ to $8 \%$. The results are summarized in Fig. 2. Our trading strategy outperforms

\footnotetext{
${ }^{22}$ For studies following this approach, see also Fleming et al. (2003); Marquering and Verbeek (2004); Han (2006); Della Corte et al. (2008).

${ }^{23}$ We estimate the conditional volatility $\hat{\sigma}_{\text {arb }, t}$ using $\operatorname{GARCH}(1,1) . r_{f}$ is the risk free rate and set to $3 \%$.
} 
all three benchmarks in terms of performance fees. For example, when $\gamma=1$ and the target volatility $\sigma_{\text {trgt }}=6 \%$, the investor is willing to pay a weekly performance fee of 66bps, 70bps, and 74bps to switch to our trading strategy from statically trading LDQ, SPY, and VXX, respectively. It is also worth noting that the performance fees reported in Fig. 2 are likely understated as the returns of the EFT funds used in the estimation are before expenses, therefore the actual performance fees might be even higher.

The economic significance is further confirmed by looking at the statistical arbitrage test developed by Hogan et al. (2004). We use the Constrained Mean (CM) statistical arbitrage test as in Hogan et al. (2004) to see whether our strategy gives rise to statistical arbitrage. Specifically, the discounted capital gain after transaction cost at the $i$ th period is assumed to be

$$
e^{-y_{0}^{i \Delta t}} C G_{i \Delta t}^{a}=\mu+\zeta i^{\lambda} x_{i}
$$

where $y_{0}^{i \Delta t}$ is the risk-free zero yield with maturity of $i \Delta t$ at zero period; $x_{i}$ is an i.i.d. $N(0,1)$ random variable; $\mu, \zeta$, and $\lambda$ are unknown parameters estimated from $C G_{i \Delta t}^{a}$ using maximum likelihood. We normalize the capital gains to be the dollar-denomination profits as in Hogan et al. (2004). In other words, we divide the aggregate capital gains by 500 since the (borrowed) initial margin for each firm is one million dollars and we have 500 firms in the portfolio. The joint hypothesis test for statistical arbitrage is $\mu>0$ and $\lambda<0$. For out-of-sample (in-sample) profits, $\widehat{\mu}=0.008(\widehat{\mu}=0.010)$ with a standard error of $0.001(0.001)$, and $\widehat{\lambda}=-2.43(\widehat{\lambda}=-0.87)$ with a standard error of $0.217(0.117)$. Therefore the null hypothesis of no statistical arbitrage is strongly rejected.

\section{Conclusion}

The rapid growth of the CDS market makes it possible to speculate on the relative pricing of a company's credit risk across a wide range of maturities. Based on a reduced-form model of credit risk, we explore mispricing in the term structure of CDS upfronts for a large number of North American companies, its economic content, and it implications. Specifically, we estimate an affine model for the term structure of CDS upfronts of a given company and identify mis-valued CDS contracts along the credit 
curve. We develop a strategy to trade market-neutral portfolios of mis-valued CDS contracts relative to our model, betting that the mis-valuation will disappear over time. The empirical analysis shows that our trading strategy generates significantly large excess returns documenting the existence of mispricing in the term structure of CDS spreads. We also find that the mispricings are positively correlated with the variance of market-wide credit and liquidity risk factors. The mispricings are more pronounced when the market is more volatile and during the financial crisis period. The fact that the extent of mispricing is related to the variance factors reinforces our concern about the pricing inefficiency of the CDS market due to market manipulation, since the market manipulation often comes with increased variance/volatility risk (see, e.g., Allen et al., 2006; Aggarwal and Wu, 2006).

To provide insights to the practical issues for practitioners interested in implementing the strategy, we provide some preliminary analysis of the strategy performance after transaction fees. Based on the Markit data, we show that the trading strategy is very profitable even after controlling for transaction costs. For most firms, both in-sample and out-of-sample Sharpe ratios are higher than one, and for some firms, they are even above two. Using rigorous statistical measures, we also show that the strategy is economically significant. We hope that our study will generate more research that benefits the long-run development of the CDS market. One interesting direction would be to check if the correlation structure of credit spreads has any impact on the mispricings, e.g., Luo et al. (2016). 


\section{Appendices}

\section{A. Formulae of $\mathbb{E}_{1}(t, u)$ and $\mathbb{E}_{2}(t, u)$}

Following Duffie et al. (2000), we consider the "Transform" and the "Extended Transform" respectively below,

$$
\begin{aligned}
\Psi\left(w, Z_{t}, t, u\right) & =\mathbb{E}^{Q}\left[\exp \left(-\int_{t}^{u} c_{0}+Z_{s} d s\right) e^{w\left(c_{0}+Z_{u}\right)} \mid \mathscr{F}_{t}\right] \\
\Phi\left(v, w, Z_{t}, t, u\right) & =\mathbb{E}^{Q}\left[\exp \left(-\int_{t}^{u} c_{0}+Z_{s} d s\right) v\left(c_{0}+Z_{u}\right) e^{w\left(c_{0}+Z_{u}\right)} \mid \mathscr{F}_{t}\right] .
\end{aligned}
$$

Proposition 1 of Duffie et al. (2000) indicates that (A1) has the following form:

$$
\Psi\left(w, Z_{t}, t, u\right)=\exp \left\{(w+t-u) c_{0}+A(t, u ; w)+B(t, u ; w) Z_{t}\right\}
$$

where $A$ and $B$ satisfy the ODEs

$$
\begin{aligned}
& \dot{B}(t, u ; w)=1+\kappa B(t, u ; w)-\frac{1}{2} B(t, u ; w)^{2} \sigma^{2}, \\
& \dot{A}(t, u ; w)=-\alpha B(t, u ; w)
\end{aligned}
$$

with boundary conditions $B(u, u)=w$ and $A(u, u)=0$, and

$$
\begin{aligned}
B(t, u ; w) & =\frac{\kappa-\frac{\left(\zeta+\kappa-w \sigma^{2}\right) e^{(u-t) \zeta}-\left(\zeta-\kappa+w \sigma^{2}\right)}{\left(\zeta+\kappa-w \sigma^{2}\right) e^{(u-t) \zeta}+\left(\zeta-\kappa+w \sigma^{2}\right)} \zeta}{\sigma^{2}} \\
A(t, u ; w) & =\alpha \int_{t}^{u} B(s, u ; w) d s \\
\zeta & =\sqrt{2 \sigma^{2}+\kappa^{2}} .
\end{aligned}
$$


Similarly, (A2) is given by

$$
\begin{aligned}
\Phi\left(v, w, Z_{t}, t, u\right) & =\left.\frac{\partial \Psi\left(\phi v+w, Z_{t}, t, u\right)}{\partial \phi}\right|_{\phi=0} \\
& =\Psi\left(w, Z_{t}, t, u\right)\left[v c_{0}+C(t, u ; v, w)+D(t, u ; v, w) Z_{t}\right]
\end{aligned}
$$

where $C$ and $D$ satisfy the ODEs

$$
\begin{aligned}
\dot{D}(t, u ; v, w) & =\kappa D(t, u ; v, w)-\frac{1}{2} D(t, u ; v, w) B(t, u ; v, w) \sigma^{2} \\
\dot{C}(t, u ; v, w) & =-\alpha D(t, u ; v, w),
\end{aligned}
$$

with boundary conditions $D(u, u ; v, w)=v$ and $C(u, u ; v, w)=0$, and

$$
\begin{aligned}
& D(t, u ; v, w)=\frac{v \zeta^{2}-\left\{\frac{\left(\zeta+\kappa-w \sigma^{2}\right) e^{(u-t) \zeta}-\left(\zeta-\kappa+w \sigma^{2}\right)}{\left(\zeta+\kappa-w \sigma^{2}\right) e^{(u-t) \zeta}+\left(\zeta-\kappa+w \sigma^{2}\right)}\right\}^{2} v \zeta^{2}}{\zeta^{2}-\left(\kappa-w \sigma^{2}\right)^{2}}, \\
& C(t, u ; v, w)=\alpha \int_{t}^{u} D(s, u ; v, w) d s .
\end{aligned}
$$

Then we have

$$
\begin{aligned}
& \mathbb{E}_{1}(t, u)=\Psi\left(0, Z_{t}, t, u\right), \\
& \mathbb{E}_{2}(t, u)=\Phi\left(1,0, Z_{t}, t, u\right) .
\end{aligned}
$$

\section{B. Unscented Kalman Filter (UKF)}

In this appendix, we briefly discuss the implementations of the unscented Kalman filter. More detailed discussions can be found in Harvey (1991) and Haykin et al. (2001).

One challenge in applying the Kalman filter to estimate the credit risk model is that the CDS spread is a nonlinear function of the state variable. One solution to this problem, the so called extended Kalman filter (EKF), is to consider a first order Taylor expansion of the measurement equation around the predicted 
state $Z_{t \mid t-1}$. Unlike the EKF, the UKF uses the exact nonlinear function $S_{t}^{\tau}\left(Z_{t}\right)$ and does not linearize the measurement equation. Instead, the UKF approximates the conditional distribution of $Z_{t}$ using a scaled unscented transformation. The essence of the UKF (Chow et al., 2007) used in this paper can be summarized briefly as follows.

For each measurement occasion $t$, a set of deterministically selected points, termed sigma points, are used to approximate the distribution of the current state ${ }^{24}$ estimates at time $t$ using a normal distribution with a mean vector $Z_{t \mid t-1}$, and a covariance matrix, which is a function in the state variance $P_{Z, t-1 \mid t-1}$ (for notational clarity, we normalize the time interval to one) and conditional variance $\mathbb{V} r_{t-1}[Z(t)]$. Sigma points are specifically selected to capture the dispersion around $Z_{t \mid t-1}$. They are then projected using the measurement function $S_{t}^{\tau}(\cdot)$, weighted, and used to update the estimates in conjunction with the newly observed measurements at time $t$ to obtain $Z_{t \mid t}$ and $P_{Z, t \mid t}$.

We start the UKF by choosing the initial values of the state variable and its variance as their steady state values:

$$
Z_{0 \mid 0}=\frac{\alpha^{P}}{\kappa^{P}}, P_{Z, 0 \mid 0}=\frac{\alpha^{P}}{2\left(\kappa^{P}\right)^{2}} \sigma^{2} .
$$

Given $Z_{t-1 \mid t-1}$ and $P_{Z, t-1 \mid t-1}$, the ex ante prediction of the state and its variance are given by

$$
\begin{aligned}
Z_{t \mid t-1} & =\frac{\alpha^{P}}{\kappa^{P}}\left(1-\exp \left(-\kappa^{P} \Delta t\right)\right)+\exp \left(-\kappa^{P} \Delta t\right) Z_{t-1 \mid t-1} \\
P_{Z, t \mid t-1} & =e^{-2 \kappa^{P} \Delta t} P_{Z, t-1 \mid t-1}+\frac{\alpha^{P} \sigma^{2}}{2\left(\kappa^{P}\right)^{2}}\left(1-e^{-\kappa^{P} \Delta t}\right)^{2}+\frac{\sigma^{2}\left(e^{-\kappa^{P} \Delta t}-e^{-2 \kappa^{P} \Delta t}\right)}{\kappa^{P}} Z_{t-1 \mid t-1} .
\end{aligned}
$$

Given an ex ante prediction of state $Z_{t \mid t-1}$, a set of three sigma points are selected as

$$
\chi_{t \mid t-1}=\left[\begin{array}{lll}
\chi_{0, t-1} & \chi_{+, t-1} & \chi_{-, t-1}
\end{array}\right],
$$

\footnotetext{
${ }^{24}$ In a typical UKF setting, both transition and measurement equations are nonlinear. Hence, the sigma points are needed to approximate the distribution of previous state estimates, in order to compute the ex ante predictions of state variables' mean and variance. However, in our paper, the transition equations are linear, so we can directly compute the ex ante predictions as in the classic Kalman Filter, and do not need the sigma points at this stage.
} 
where

$$
\begin{aligned}
& \chi_{0, t-1}=Z_{t \mid t-1}, \\
& \chi_{+, t-1}=Z_{t \mid t-1}+\sqrt{(1+\rho)}\left(\exp \left(-\kappa^{P} \Delta t\right) \sqrt{P_{Z, t-1 \mid t-1}}+\sqrt{\operatorname{Var}_{t-1}[Z(t)]}\right), \\
& \chi_{-, t-1}=Z_{t \mid t-1}-\sqrt{(1+\rho)}\left(\exp \left(-\kappa^{P} \Delta t\right) \sqrt{P_{Z, t-1 \mid t-1}}+\sqrt{\operatorname{Var}_{t-1}[Z(t)]}\right) .
\end{aligned}
$$

The term $\rho$ is a scaling constant and given by

$$
\rho=\phi^{2}(1+\kappa)-1
$$

where $\phi$ and $\kappa$ are user-specified constants. In this paper, we choose $\phi=0.001$ and $\kappa=2$. Since the values of these constants are not critical in our analysis, we do not provide more detailed discussions for brevity. Curious readers are referred to Chow et al. (2007) or Chapter 7 in Haykin et al. (2001) for details.

$\chi_{t \mid t-1}$ is propagated through the nonlinear measurement function $U(\cdot, \tau)$ (i.e. nonlinear transformation of the sigma points through measurement function)

$$
\mathrm{S}_{t \mid t-1}=U\left(\chi_{t \mid t-1}, \tau\right)
$$

where the dimension of $S_{t \mid t-1}$ is $9 \times 3$. We define the set of weights for covariance matrix estimates as

$$
W^{(c)}=\operatorname{diag}\left[\frac{\rho}{1+\rho}+1-\phi^{2}+2, \frac{1}{2(1+\rho)}, \frac{1}{2(1+\rho)}\right] ;
$$

and the weights for mean estimates as $W^{(m)}=\left[\begin{array}{lll}\frac{\rho}{1+\rho} & \frac{1}{2(1+\rho)} & \frac{1}{2(\omega+\rho)}\end{array}\right]^{\top}$.

Predicted measurements and the associated covariance matrix are computed as

$$
\begin{aligned}
S_{t \mid t-1} & =\mathrm{S}_{t \mid t-1} W^{(m)}, \\
P_{y_{t}} & =\left[\mathrm{S}_{t \mid t-1}-\mathbf{1}_{1 \times 3} \otimes S_{t \mid t-1}\right] W^{(c)}\left[\mathrm{S}_{t \mid t-1}-\mathbf{1}_{1 \times 3} \otimes S_{t \mid t-1}\right]^{\top}+V, \\
P_{Z_{t}, y_{t}} & =\left[\chi_{t \mid t-1}-\mathbf{1}_{1 \times 3} \otimes Z_{t \mid t-1}\right] W^{(c)}\left[\mathrm{S}_{t \mid t-1}-\mathbf{1}_{1 \times 3} \otimes S_{t \mid t-1}\right]^{\top},
\end{aligned}
$$


where $V=\operatorname{diag}\left[v_{1}^{2}, v_{2}^{2}, \cdots, v_{30}^{2}\right]_{9 \times 9}$.

Finally, the discrepancy between model prediction and actual observations is weighted by a Kalman gain $\Xi_{t}$ function to yield ex post state and variance estimates as

$$
\begin{aligned}
Z_{t \mid t} & =Z_{t \mid t-1}+\Xi_{t}\left(S_{t}-S_{t \mid t-1}\right), \\
P_{Z, t \mid t} & =P_{Z, t \mid t-1}-\Xi_{t} P_{y_{t}} \Xi_{t}^{\top},
\end{aligned}
$$

where $\Xi_{t}=P_{Z_{t}, y_{t}} P_{y_{t}}^{-1}$.

\section{Closed-form Formulae of $H_{1}$ and $H_{2}$}

In this appendix, we derive the closed-form formulae for $H_{1}\left(Z_{t}, \tau\right)=\left.\frac{\partial U(Z, \tau)}{\partial Z}\right|_{Z=Z_{t}}$, and $H_{2}\left(Z_{t}, \tau\right)=$ $\left.\frac{\partial^{2} U(Z, \tau)}{\partial Z^{2}}\right|_{Z=Z_{t}}$. Given (8), we have

$H_{1}\left(Z_{t}, \tau\right)=\frac{y \sum_{i=1}^{4 \tau} P\left(t, t+\frac{2 i-1}{8}\right) \frac{\partial \mathbb{E}_{2}\left(t, t+\frac{2 i-1}{8}\right)}{\partial Z_{t}}-C\left\{\sum_{i=1}^{4 \tau} P\left(t, t+\frac{i}{4}\right) \frac{\partial \mathbb{E}_{1}\left(t, t+\frac{2 i-1}{8}\right)}{\partial Z_{t}}+\frac{1}{8} \sum_{i=1}^{4 \tau} P\left(t, t+\frac{2 i-1}{8}\right) \frac{\partial \mathbb{E}_{2}\left(t, t+\frac{2 i-1}{8}\right)}{\partial Z_{t}}\right\}}{4}$

$H_{2}\left(Z_{t}, \tau\right)=\frac{y \sum_{i=1}^{4 \tau} P\left(t, t+\frac{2 i-1}{8}\right) \frac{\partial^{2} \mathbb{E}_{2}\left(t, t+\frac{2 i-1}{8}\right)}{\partial Z_{t}^{2}}-C\left\{\sum_{i=1}^{4 \tau} P\left(t, t+\frac{i}{4}\right) \frac{\partial^{2} \mathbb{E}_{1}\left(t, t+\frac{2 i-1}{8}\right)}{\partial Z_{t}^{2}}+\frac{1}{8} \sum_{i=1}^{4 \tau} P\left(t, t+\frac{2 i-1}{8}\right) \frac{\partial^{2} \mathbb{E}_{2}\left(t, t+\frac{2 i-1}{8}\right)}{\partial Z_{t}^{2}}\right\}}{4}$ 
Therefore we just need to derive $\frac{\partial \mathbb{E}_{1}(t, u)}{\partial Z_{t}}, \frac{\partial \mathbb{E}_{2}(t, u)}{\partial Z_{t}}, \frac{\partial^{2} \mathbb{E}_{1}(t, u)}{\partial Z_{t}^{2}}$, and $\frac{\partial^{2} \mathbb{E}_{2}(t, u)}{\partial Z_{t}^{2}}$.

$$
\begin{aligned}
\frac{\partial \mathbb{E}_{1}(t, u)}{\partial Z_{t}} & =\frac{\partial \Psi\left(0, Z_{t}, t, u\right)}{\partial Z_{t}} \\
& =\Psi\left(0, Z_{t}, t, u\right) B(t, u ; 0) \\
& =\mathbb{E}_{1}(t, u) B(t, u ; 0), \\
\frac{\partial \mathbb{E}_{2}(t, u)}{\partial Z_{t}} & =\frac{\partial \Phi\left(1,0, Z_{t}, t, u\right)}{\partial Z_{t}} \\
& =\frac{\partial \Psi\left(0, Z_{t}, t, u\right)\left[c_{0}+C(t, u ; 1,0)+D(t, u ; 1,0) Z_{t}\right]}{\partial Z_{t}} \\
& =\left\{\left[c_{0}+C(t, u ; 1,0)+D(t, u ; 1,0) Z_{t}\right] B(t, u ; 0)+D(t, u ; 1,0)\right\} \Psi\left(0, Z_{t}, t, u\right) \\
& =\left\{\mathbb{E}_{2}(t, u) B(t, u ; 0)+\mathbb{E}_{1}(t, u) D(t, u ; 1,0),\right. \\
\frac{\partial^{2} \mathbb{E}_{1}(t, u)}{\partial Z_{t}^{2}} & =\frac{\partial \mathbb{E}_{1}(t, u)}{\partial Z_{t}} B(t, u ; 0), \\
\frac{\partial^{2} \mathbb{E}_{2}(t, u)}{\partial Z_{t}^{2}} & =\frac{\partial \mathbb{E}_{2}(t, u)}{\partial Z_{t}} B(t, u ; 0)+\frac{\partial \mathbb{E}_{1}(t, u)}{\partial Z_{t}} D(t, u ; 1,0) .
\end{aligned}
$$

\section{Results using GFI CDS Data}

The CDS curve data from GFI are similar to those from Markit but with fewer maturities: each curve consists of seven points for tenors from one to five-year, seven-year and 10-year. Same as in Markit data, we use CDS on senior unsecured issues with MR clauses. We require the firms to have complete data coverage over the seven years (from Jan 2005 to Oct 2011) and end up with 200 firms in the sample with daily CDS premia on the seven tenors. All the CDS premia are converted to upfronts using ISAD standard CDS converter, assuming the fixed coupon rate $C=100 \mathrm{bps}$ and recovery rate $40 \%$ (See ISDA, 2009). We bootstrap zero yields from Constant Maturity Treasury (CMT) yields from release Fed H.15. The default-free discount factor used in our upfront pricing model is prices of zero coupon bonds computed using these zero yields.

In a preliminary analysis of the GFI data, we find that the one-factor model fail to adequately capture the dynamic of the upfront term structures. To better fit the data, we develop a two-factor upfront model. 
The details of the model can be found in the online appendix of this paper. Given the two-factor, a two-factor strategy is also developed to ensure the portfolios of CDS contract are immune to the first two orders of the changes of two factors. The online appendix also has the details.

Similar to the Markit data case, the two-factor model is estimated only using the first half of the full sample (January 2005 to June 2008); then based on the estimated model, the two-factor strategy is implemented on the second half of the full sample (July 2008 to October 2011).

Analogous to Fig. 1, the three panels in Fig. A1 show the histograms and the cumulative path of the aggregate returns based on the GFI data. The annualized return is $25.2 \%$ and annualized Sharpe ratio is 15.6. The trading strategy again identifies the mispricing in the GFI data. While the mispricing persists regardless of the financial crisis, in the GFI data the observation of more severe mispricing during the financial crisis period is less evident. Indeed, without the financial crisis period, the annualized return only reduces by two percent to $22.7 \%$, and the annualized Sharpe ratio only increases by slightly more than one to 17. The analogy of Table 5 is presented in Table A1. The same conclusion about the nonlinear risk factors holds in the GFI data with lower adjusted $R^{2} \mathrm{~s}$ and a more (less) significant coefficient for CRDTSPRD $\left(\mathrm{CRDTSPRD}^{2}\right)$ in the second (third) regression. 


\section{References}

Abrantes-Metz, R. M., Kraten, M., Metz, A. D., and Seow, G. S. (2012). Libor manipulation? Journal of Banking \& Finance, 36(1):136-150.

Aggarwal, R. K. and Wu, G. (2006). Stock market manipulations. The Journal of Business, 79(4):19151953.

Allen, F., Litov, L., and Mei, J. (2006). Large investors, price manipulation, and limits to arbitrage: An anatomy of market corners. Review of Finance, 10(4):645-693.

Arakelyan, A. and Serrano, P. (2012). Liquidity in credit default swap markets. Working paper, Universidad Carlos III de Madrid.

Avellaneda, M. and Lee, J.-H. (2010). Statistical arbitrage in the us equities market. Quantitative Finance, 10(7):761-782.

Bali, T., Heidari, M., and Wu, L. (2009). Predictability of interest rates and interest-rate portfolios. Journal of Business \& Economic Statistics, 27(4):517-527.

Berndt, A., Douglas, R., Duffie, D., Ferguson, M., and Schranz, D. (2008). Measuring Default Risk Premia from Default Swap Rates and EDFs. Working paper, Carnegie Mellon University.

Biswas, G., Nikolova, S., and Stahel, C. W. (2014). The transaction costs of trading corporate credit. Available at SSRN 2532805.

Blanco, R., Brennan, S., and Marsh, I. (2005). An empirical analysis of the dynamic relationship between investment-grade bonds and credit default swaps. Journal of Finance, 60(5):2255-81.

Bloomfield, R. and Michaely, R. (2004). Risk or mispricing? from the mouths of professionals. Financial Management, pages 61-81.

Bondarenko, O. (2003). Statistical arbitrage and securities prices. Review of Financial Studies, 16(3):875-919. 
Brave, S. and Butters, R. A. (2011). Monitoring financial stability: a financial conditions index approach. Economic Perspectives, (QI):22-43.

Brave, S. and Butters, R. A. (2012). Diagnosing the financial system: Financial conditions and financial stress. International Journal of Central Banking, 8(2):191-239.

Brennan, M. J. and Wang, A. W. (2010). The mispricing return premium. The Review of Financial Studies, 23(9):3437-3468.

Brunnermeier, M. K. and Pedersen, L. H. (2009). Funding liquidity and market liquidity. Review of Financial Studies, 22(2201-2238):6.

Burne, K. (2015). Banks near pact on swaps suit. The Wall Street Journal, September 12.

Burne, K. (2016). Swaps payout is a windfall for funds. The Wall Street Journal, January 11.

Chen, R.-R., Cheng, X., and Wu, L. (2013). Dynamic interactions between interest-rate and credit risk: Theory and evidence on the credit default swap term structure. Review of Finance, 17(1):403-441.

Cheridito, P., Filipović, D., and Kimmel, R. (2007). Market price of risk specifications for affine models: Theory and evidence. Journal of Financial Economics, 83(1):123-170.

Chow, S., Ferrer, E., and Nesselroade, J. (2007). An Unscented Kalman Filter Approach to the Estimation of Nonlinear Dynamical Systems Models. MULTIVARIATE BEHAVIORAL RESEARCH, 42(2):283321.

Cui, C., Liu, H., and Zhang, Y. (2013). On credit spread change of chinese corporate bonds: credit risk or asset allocation effect? China Finance Review International, 3(3):250-263.

Dai, Q. and Singleton, K. (2003). Term structure dynamics in theory and reality. Review of Financial Studies, 16(3):631-678.

Della Corte, P., Sarno, L., and Thornton, D. L. (2008). The expectation hypothesis of the term structure of very short-term rates: Statistical tests and economic value. Journal of Financial Economics, 89(1):158-174. 
Du, S. and Zhu, H. (2015). Are CDS auctions biased and inefficient? Available at SSRN 1804610.

Duan, J. and Simonato, J. (1999). Estimating and testing exponential-affine term structure models by kalman filter. Review of Quantitative Finance and Accounting, 13(2):111-135.

Duffee, G. (1999). Estimating the price of default risk. Review of Financial Studies, 12(1):197-226.

Duffie, D. (2010). Presidential address: asset price dynamics with slow-moving capital. The Journal of finance, 65(4):1237-1267.

Duffie, D., Pan, J., and Singleton, K. (2000). Transform analysis and asset pricing for affine jumpdiffusions. Econometrica, pages 1343-1376.

Duffie, D., Pedersen, L., and Singleton, K. (2003). Modeling sovereign yield spreads: A case study of Russian debt. Journal of Finance, pages 119-159.

Duffie, D. and Singleton, K. (1997). An econometric model of the term structure of interest-rate swap yields. Journal of finance, pages 1287-1321.

Duffie, D. and Singleton, K. (1999). Modeling term structures of defaultable bonds. Review of Financial Studies, 12(4):687-720.

Feller, W. (1951). Two singular diffusion problems. Annals of mathematics, pages 173-182.

Filipović, D. and Trolle, A. B. (2013). The term structure of interbank risk. Journal of Financial Economics, 109(3):707-733.

Fleckenstein, M., Longstaff, F. A., and Lustig, H. (2014). The tips-treasury bond puzzle. the Journal of Finance, 69(5):2151-2197.

Fleming, J., Kirby, C., and Ostdiek, B. (2001). The economic value of volatility timing. The Journal of Finance, 56(1):329-352.

Fleming, J., Kirby, C., and Ostdiek, B. (2003). The economic value of volatility timing using "realized" volatility. Journal of Financial Economics, 67(3):473-509. 
Fouquau, J. and Spieser, P. K. (2015). Statistical evidence about LIBOR manipulation: A “sherlock holmes" investigation. Journal of Banking \& Finance, 50:632-643.

Gibson, M. S. (2007). Credit derivatives and risk management. Economic Review, (Q4):25-41.

Han, Y. (2006). Asset allocation with a high dimensional latent factor stochastic volatility model. Review of Financial Studies, 19(1):237-271.

Harvey, A. (1991). Forecasting, structural time series models and the Kalman filter. Cambridge Univ $\operatorname{Pr}$.

Haykin, S. et al. (2001). Kalman Filtering and Neural Networks. Wiley Chichester.

Hirshleifer, D. (2001). Investor psychology and asset pricing. The Journal of Finance, 56(4):1533-1597.

Hogan, S., Jarrow, R., Teo, M., and Warachka, M. (2004). Testing market efficiency using statistical arbitrage with applications to momentum and value strategies. Journal of Financial economics, 73(3):525-565.

Hu, G. X., Pan, J., and Wang, J. (2013). Noise as information for illiquidity. The Journal of Finance, 68(6):2341-2382.

ISDA (2009). ISDA standard CDS converter specification. Technical report, www.isda.com.

Jarrow, R., Li, H., Liu, S., and Wu, C. (2010). Reduced-form valuation of callable corporate bonds: Theory and evidence. Journal of Financial Economics, 95(2):227-248.

Jarrow, R., Teo, M., Tse, Y. K., and Warachka, M. (2012). An improved test for statistical arbitrage. Journal of Financial Markets, 15(1):47-80.

Jarrow, R. A. (1992). Market manipulation, bubbles, corners, and short squeezes. Journal of financial and Quantitative Analysis, 27(3):311-336.

Jarrow, R. A. (2012). Problems with using CDS to infer default probabilities. Journal of Fixed Income, 21(4):6. 
Jarrow, R. A. and Larsson, M. (2012). The meaning of market efficiency. Mathematical Finance, 22(1):1-30.

Levine, R. (2012). The governance of financial regulation: reform lessons from the recent crisis. International Review of Finance, 12(1):39-56.

Li, H., Wu, C., and Shi, J. (2017). Estimating liquidity premium of corporate bonds using the spread information in on-and off-the-run treasury securities. China Finance Review International, 7(2):134162.

Longstaff, F., Mithal, S., and Neis, E. (2005). Corporate yield spreads: Default risk or liquidity? New evidence from the credit default swap market. Journal of Finance, 55(5):2213-2253.

Luo, C., Li, M., and Ouyang, Z. (2016). An empirical study on the correlation structure of credit spreads based on the dynamic and pair copula functions. China Finance Review International, 6(3):284-303.

Marquering, W. and Verbeek, M. (2004). The economic value of predicting stock index returns and volatility. Journal of Financial and Quantitative Analysis, 39(02):407-429.

Mayordomo, S., Peña, J. I., and Romo, J. (2014). Testing for statistical arbitrage in credit derivatives markets. Journal of Empirical Finance, 26:59-75.

McConnell, P. J. (2013). Systemic operational risk: the LIBOR manipulation scandal. Journal of Operational Risk, 8(3):59-99.

Mitchell, M., Pedersen, L. H., and Pulvino, T. (2007). Slow moving capital. American Economic Review, 97(2):215-220.

Mollenkamp, C. and Whitehouse, M. (2008). Study casts doubt on key rate: WSJ analysis suggests banks may have reported flawed interest data for libor. The Wall Street Journal, May.

Mueller, P., TAHBAZ-SALEHI, A., and Vedolin, A. (2017). Exchange rates and monetary policy uncertainty. The Journal of Finance, 72(3):1213-1252. 
Norden, L. and Weber, M. (2004). Informational efficiency of credit default swap and stock markets: The impact of credit rating announcements. Journal of Banking and Finance, 28(11):2813-2843.

Oehmke, M. and Zawadowski, A. (2016). The anatomy of the CDS market. The Review of Financial Studies, 30(1):80-119.

Pan, J. and Singleton, K. (2008). Default and recovery implicit in the term structure of sovereign CDS spreads. The Journal of Finance, 63(5):2345-2384.

Phillips, S. M. and Smith, C. W. (1980). Trading costs for listed options: The implications for market efficiency. Journal of financial economics, 8(2):179-201.

Pole, A. (2007). Statistical arbitrage: algorithmic trading insights and techniques. John Wiley \& Sons.

Sercu, P. and Wu, X. (1997). The information content in bond model residuals: An empirical study on the belgian bond market. Journal of Banking \& Finance, 21(5):685-720.

Stambaugh, R. F. and Yuan, Y. (2016). Mispricing factors. The Review of Financial Studies, 30(4):12701315.

Stulz, R. M. (2010). Credit default swaps and the credit crisis. The Journal of Economic Perspectives, 24(1):73.

van Deventer, D. (2015). CDS trading volume for 1,239 reference names from 2010 to 2014. Kamakura Corporate Blog, January.

Whistler, M. (2004). Trading pairs: capturing profits and hedging risk with statistical arbitrage strategies, volume 216. John Wiley \& Sons.

Zhang, F. X. (2008). Market expectations and default risk premium in credit default swap prices. The Journal of Fixed Income, 18(1):37-55.

Zhang, G. (2009). Informational efficiency of credit default swap and stock markets: The impact of adverse credit events. International Review of Accounting, Banking and Finance, 1. 
Zhang, G. and Zhang, S. (2013). Information efficiency of the U.S. credit default swap market: Evidence from earnings surprises. Journal of Financial Stability, 9(4):720 - 730. Re-examining the role of the state in the financial sector. 
Table 1: Summary of Variance Ratios

This table shows the first quarter (Q1), median, third quarter (Q3), and mean of the of the variance ratios, which are the proportion of variations of the actual upfronts explained by our upfront pricing model, at nine maturities (1yr, 2yr, 3yr, 5yr, 7yr, 10yr, 15yr, 20yr, and 30yr) as well as the average value (Ave.) across maturities

\begin{tabular}{|c|c|c|c|c|c|c|c|c|}
\hline & \multicolumn{4}{|c|}{ Full sample } & \multicolumn{4}{|c|}{ First sub-sample } \\
\hline & Q1 & Median & Q3 & Mean & Q1 & Median & Q3 & Mean \\
\hline $1 \mathrm{yr}$ & 0.76 & 0.86 & 0.92 & 0.81 & 0.71 & 0.84 & 0.91 & 0.75 \\
\hline $2 \mathrm{yr}$ & 0.88 & 0.93 & 0.96 & 0.89 & 0.86 & 0.92 & 0.96 & 0.86 \\
\hline $3 \mathrm{yr}$ & 0.95 & 0.97 & 0.98 & 0.95 & 0.94 & 0.97 & 0.98 & 0.93 \\
\hline $5 y r$ & 1.00 & 1.00 & 1.00 & 1.00 & 1.00 & 1.00 & 1.00 & 1.00 \\
\hline $7 \mathrm{yr}$ & 0.96 & 0.98 & 0.99 & 0.97 & 0.95 & 0.98 & 0.99 & 0.95 \\
\hline $10 \mathrm{yr}$ & 0.90 & 0.96 & 0.98 & 0.92 & 0.86 & 0.94 & 0.97 & 0.89 \\
\hline $15 \mathrm{yr}$ & 0.85 & 0.93 & 0.97 & 0.88 & 0.76 & 0.86 & 0.93 & 0.81 \\
\hline $20 \mathrm{yr}$ & 0.80 & 0.91 & 0.96 & 0.84 & 0.68 & 0.81 & 0.90 & 0.76 \\
\hline $30 \mathrm{yr}$ & 0.75 & 0.89 & 0.94 & 0.81 & 0.61 & 0.74 & 0.85 & 0.70 \\
\hline Ave. & 0.87 & 0.93 & 0.96 & 0.90 & 0.82 & 0.89 & 0.94 & 0.85 \\
\hline
\end{tabular}


Table 2: Summary of Parameter Estimates

This table reports the first quarter (Q1), median, third quarter (Q3), and mean of the parameter estimates of the 500 firms used in our empirical analysis. Panel (a) is the summary of parameter estimates using the full sample (Jan2005-Oct2011), Panel (b) is the summary of parameter estimates using the first half of the sample (Jan2005Jun2006)

(a) Full sample (Jan2005 - Oct2011)

\begin{tabular}{|c|c|c|c|c|c|c|}
\hline & $\alpha$ & $\kappa$ & $\sigma$ & $\alpha^{P}$ & $\kappa^{P}$ & $1-y$ \\
\hline Q1 & 0.001 & -0.306 & 0.115 & 0.003 & 0.353 & 0.472 \\
\hline Median & 0.002 & -0.189 & 0.143 & 0.017 & 0.914 & 0.572 \\
\hline Q3 & 0.004 & -0.088 & 0.178 & 0.048 & 1.601 & 0.834 \\
\hline Mean & 0.004 & -0.221 & 0.154 & 0.062 & 1.416 & 0.627 \\
\hline \multicolumn{7}{|c|}{ (b) First sub-sample (Jan2005-Jun2008) } \\
\hline & $\alpha$ & $\kappa$ & $\sigma$ & $\alpha^{P}$ & $\kappa^{P}$ & $1-y$ \\
\hline Q1 & 0.001 & -0.510 & 0.143 & 0.005 & 0.714 & 0.553 \\
\hline Median & 0.002 & -0.392 & 0.173 & 0.026 & 2.552 & 0.841 \\
\hline Q3 & 0.003 & -0.250 & 0.206 & 0.082 & 4.422 & 0.949 \\
\hline Mean & 0.004 & -0.386 & 0.175 & 0.093 & 7.172 & 0.738 \\
\hline
\end{tabular}


Table 3: Median Adjusted Long Term Mean of Default Intensity in Different Ratings and Sectors

This table reports the median of the adjusted long term mean of the default intensities $\left(c_{0}\right.$ plus the long term mean of $Z_{t}$ under measure $P, \alpha^{P} / \kappa^{P}$ ) of different ratings and sectors. Panel (a) is based on the full sample (Jan2005Dec2011) estimates, Panel (b) is based the first half of the sample (Jan2005-Jun2008) estimates. The last column (the last row), except the last cell, is the median over each row (column). The last cell at the right-lower conner is the median over all firms.

(a) Full sample (Jan2005 - Dec2011)

\begin{tabular}{lccccccc}
\hline & AA & A & BBB & BB & B & CCC & Median \\
\hline BM & & 0.000 & 0.009 & 0.011 & 0.067 & 0.053 & 0.011 \\
CG & 0.007 & & 0.013 & 0.031 & 0.039 & 0.044 & 0.031 \\
CS & 0.005 & & 0.006 & 0.017 & 0.019 & 0.033 & 0.017 \\
Fin & 0.013 & & 0.022 & 0.024 & & 0.000 & 0.017 \\
HC & & & 0.013 & 0.026 & 0.029 & 0.017 & 0.021 \\
Ind & 0.009 & & 0.014 & & & 0.001 & 0.009 \\
OG & & 0.017 & 0.009 & 0.029 & & 0.050 & 0.023 \\
Tec & & & 0.009 & 0.003 & 0.028 & 0.048 & 0.019 \\
Tel & 0.003 & & 0.006 & 0.024 & & & 0.006 \\
Uti & 0.012 & 0.011 & 0.015 & & 0.046 & 0.012 & 0.012 \\
Median & 0.008 & 0.011 & 0.011 & 0.024 & 0.034 & 0.033 & 0.013 \\
\hline
\end{tabular}

(b) First sub-sample (Jan2005-Jun2008)

\begin{tabular}{lccccccc}
\hline & AA & A & BBB & BB & B & CCC & Median \\
\hline BM & & 0.003 & 0.008 & 0.018 & 0.007 & 0.008 & 0.008 \\
CG & 0.005 & & 0.012 & 0.009 & 0.015 & 0.033 & 0.012 \\
CS & 0.002 & & 0.002 & 0.002 & 0.005 & 0.023 & 0.002 \\
Fin & 0.006 & & 0.006 & 0.004 & & & 0.006 \\
HC & & & 0.004 & 0.016 & 0.044 & 0.009 & 0.012 \\
Ind & 0.013 & & 0.011 & & & 0.011 & 0.011 \\
OG & & 0.012 & 0.009 & 0.007 & & 0.009 & 0.009 \\
Tec & & & 0.005 & 0.000 & 0.021 & & 0.005 \\
Tel & 0.005 & & 0.005 & 0.007 & & & 0.005 \\
Uti & 0.018 & 0.016 & 0.006 & & 0.007 & 0.007 & 0.007 \\
Median & 0.005 & 0.012 & 0.006 & 0.007 & 0.011 & 0.009 & 0.008 \\
\hline
\end{tabular}


Table 4: Median Recovery Rate in Different Ratings and Sectors

This table reports the median of the recovery rates in different ratings and sectors. Panel (a) is based on the full sample (Jan2005-Oct2011) estimates, Panel (b) is based the first half of the sample (Jan2005-Jun2008) estimates. The last column (the last row), except the last cell, is the median over each row (column). The last cell at the right-lower conner is the median over all firms.

(a) Full sample (Jan2005 - Oct2011)

\begin{tabular}{lccccccc}
\hline & AA & A & BBB & BB & B & CCC & Median \\
\hline BM & & 0.925 & 0.709 & 0.421 & 0.471 & 0.467 & 0.471 \\
CG & 0.880 & & 0.794 & 0.460 & 0.410 & 0.416 & 0.460 \\
CS & 0.869 & & 0.755 & 0.501 & 0.456 & 0.412 & 0.501 \\
Fin & 0.571 & & 0.499 & 0.416 & & 0.427 & 0.463 \\
HC & & & 0.831 & 0.547 & 0.513 & 0.436 & 0.530 \\
Ind & 0.888 & & 0.684 & & & 0.408 & 0.684 \\
OG & & 0.931 & 0.532 & 0.468 & & 0.401 & 0.500 \\
Tec & & & 0.726 & 0.598 & 0.389 & 0.401 & 0.499 \\
Tel & 0.871 & & 0.647 & 0.472 & & & 0.647 \\
Uti & 0.905 & 0.857 & 0.543 & & 0.451 & 0.272 & 0.543 \\
Median & 0.876 & 0.925 & 0.697 & 0.470 & 0.454 & 0.412 & 0.572 \\
\hline
\end{tabular}

(b) First sub-sample (Jan2005-Jun2008)

\begin{tabular}{lccccccc}
\hline & AA & A & BBB & BB & B & CCC & Median \\
\hline BM & & 0.940 & 0.919 & 0.503 & 0.459 & 0.508 & 0.508 \\
CG & 0.959 & & 0.958 & 0.556 & 0.277 & 0.456 & 0.556 \\
CS & 0.899 & & 0.853 & 0.457 & 0.392 & 0.471 & 0.471 \\
Fin & 0.837 & & 0.778 & 0.505 & & 0.514 & 0.646 \\
HC & & & 0.900 & 0.598 & 0.671 & 0.392 & 0.634 \\
Ind & 0.958 & & 0.849 & & & 0.522 & 0.849 \\
OG & & 0.973 & 0.947 & 0.389 & & 0.225 & 0.668 \\
Tec & & & 0.945 & 0.737 & 0.338 & 0.497 & 0.617 \\
Tel & 0.896 & & 0.607 & 0.309 & & & 0.607 \\
Uti & 0.975 & 0.972 & 0.872 & & 0.012 & 0.147 & 0.872 \\
Median & 0.928 & 0.972 & 0.886 & 0.504 & 0.365 & 0.471 & 0.845 \\
\hline
\end{tabular}


Table 5: Results of the return factor regressions

This table reports the OLS coefficients of the return factor regressions. Newey-West standard errors are in brackets. $* * *, * * *$ represent the significance levels at $1 \%, 5 \%$, and $10 \%$, respectively. The LHS variable is the aggregate returns of the trading strategy from in-sample and out-of-sample. Besides the control variables, USCDS, NFCI, and MES, the RHS variables include CRDTSPRD and NOISE for regression (1); CRDTSPRD, CRDTSPRD ${ }^{2}$, NOISE, and NOISE ${ }^{2}$ for regression (2); CRDTSPRD ${ }^{2}$ and NOISE$^{2}$ for regression (3). The upper panel reports full sample (in-sample Jan 2005 - Oct 2011) results, the middle panel reports in-sample (Jul 2008 - Oct 2011) results, and the lower panel reports out-of-sample (Jul 2008 - Oct 2011) results. To make the coefficients comparable in magnitude, CRDTSPRD ${ }^{2}$ and NOISE ${ }^{2}$ are multiplied by 1000 and 100,000 in the regressions.

\begin{tabular}{|c|c|c|c|c|c|c|c|c|c|c|}
\hline \multirow{4}{*}{ 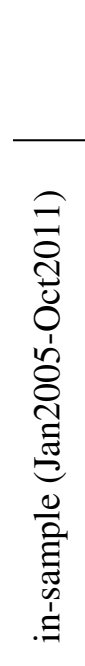 } & \multirow{2}{*}{ (1) } & \multirow{2}{*}{$\begin{array}{c}\text { Const. } \\
0.006^{* * *} \\
(0.00)\end{array}$} & \multicolumn{3}{|c|}{ CRDTSPRDCRDTSPRDANOISE } & \multirow{2}{*}{$\begin{array}{c}\text { NOISE }^{2} \\
-\end{array}$} & \multirow{2}{*}{$\begin{array}{c}\text { US CDS } \\
0.138 \\
(0.50)\end{array}$} & \multirow{2}{*}{$\begin{array}{c}\text { NFCI } \\
-0.314 \\
(0.27)\end{array}$} & \multirow{2}{*}{$\begin{array}{c}\text { MES } \\
0.562 \\
(0.64)\end{array}$} & \multirow{2}{*}{$\begin{array}{c}\text { Adj. } \\
\mathrm{R}^{2}(\%) \\
0.68\end{array}$} \\
\hline & & & $\begin{array}{l}0.630 \\
(0.51)\end{array}$ & - & $\begin{array}{l}5.083 \\
(4.44)\end{array}$ & & & & & \\
\hline & (2) & $\begin{array}{c}0.005^{* * *} \\
(0.00)\end{array}$ & $\begin{array}{c}-0.122 \\
(0.33)\end{array}$ & $\begin{array}{c}0.400^{* * *} \\
(0.10)\end{array}$ & $\begin{array}{c}-0.929 \\
(3.41)\end{array}$ & $\begin{array}{c}0.911^{* * *} \\
(0.24)\end{array}$ & $\begin{array}{l}0.037 \\
(0.33)\end{array}$ & $\begin{array}{r}-0.408 \\
(0.28)\end{array}$ & $\begin{array}{l}0.150 \\
(0.62)\end{array}$ & 16.45 \\
\hline & (3) & $\begin{array}{c}0.006^{* * *} \\
(0.00)\end{array}$ & - & $\begin{array}{c}0.381^{* * *} \\
(0.07)\end{array}$ & - & $\begin{array}{c}0.890^{* * *} \\
(0.26)\end{array}$ & $\begin{array}{l}0.031 \\
(0.33)\end{array}$ & $\begin{array}{c}-0.455 \\
(0.28)\end{array}$ & $\begin{array}{l}0.168 \\
(0.61)\end{array}$ & 17.22 \\
\hline 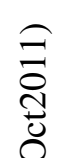 & (1) & $\begin{array}{c}0.011^{\text {*** }} \\
(0.00)\end{array}$ & $\begin{array}{l}2.475 \\
(1.62)\end{array}$ & - & $\begin{array}{l}17.593 \\
(13.55)\end{array}$ & - & $\begin{array}{l}1.011 \\
(1.41)\end{array}$ & $\begin{array}{r}-1.019 \\
(2.23)\end{array}$ & $\begin{array}{l}0.427 \\
(1.62)\end{array}$ & 4.78 \\
\hline $\begin{array}{l}\infty \\
\stackrel{0}{8} \\
\stackrel{1}{\Xi}\end{array}$ & (2) & $\begin{array}{c}0.009^{* * *} \\
(0.00)\end{array}$ & $\begin{array}{c}-0.842 \\
(0.83)\end{array}$ & $\begin{array}{c}1.682^{* * *} \\
(0.25)\end{array}$ & $\begin{array}{r}-7.402 \\
(9.88)\end{array}$ & $\begin{array}{c}3.029^{* * *} \\
(0.54)\end{array}$ & $\begin{array}{l}0.685 \\
(1.26)\end{array}$ & $\begin{array}{l}0.314 \\
(2.60)\end{array}$ & $\begin{array}{l}1.205 \\
(1.91)\end{array}$ & 38.72 \\
\hline 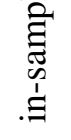 & (3) & $\begin{array}{c}0.009^{* * *} \\
(0.00)\end{array}$ & - & $\begin{array}{c}1.541^{* * *} \\
(0.29)\end{array}$ & - & $\begin{array}{c}2.829^{* * *} \\
(0.63)\end{array}$ & $\begin{array}{l}0.663 \\
(1.26)\end{array}$ & $\begin{array}{c}-0.227 \\
(2.34)\end{array}$ & $\begin{array}{l}1.085 \\
(1.83)\end{array}$ & 38.89 \\
\hline 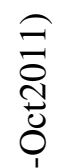 & (1) & $\begin{array}{c}0.012^{* * *} \\
(0.00)\end{array}$ & $\begin{array}{c}3.910^{* *} \\
(1.78)\end{array}$ & - & $\begin{array}{l}28.887^{*} \\
(15.35)\end{array}$ & - & $\begin{array}{l}2.245 \\
(2.12)\end{array}$ & $\begin{array}{r}-1.666 \\
(2.66)\end{array}$ & $\begin{array}{c}-0.233 \\
(1.87)\end{array}$ & 11.54 \\
\hline $\begin{array}{l}\stackrel{\Xi}{0} \\
\stackrel{0}{\Xi} \\
\stackrel{0}{\Xi}\end{array}$ & (2) & $\begin{array}{c}0.009^{* * *} \\
(0.00)\end{array}$ & $\begin{array}{l}0.167 \\
(1.00)\end{array}$ & $\begin{array}{c}1.904^{* * *} \\
(0.28)\end{array}$ & $\begin{array}{c}0.831 \\
(15.02)\end{array}$ & $\begin{array}{c}3.392^{* * *} \\
(0.76)\end{array}$ & $\begin{array}{l}1.881 \\
(1.59)\end{array}$ & $\begin{array}{r}-0.168 \\
(2.40)\end{array}$ & $\begin{array}{l}0.657 \\
(1.86)\end{array}$ & 40.38 \\
\hline 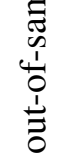 & (3) & $\begin{array}{c}0.009^{* * *} \\
(0.00)\end{array}$ & - & $\begin{array}{c}1.931^{* * *} \\
(0.25)\end{array}$ & - & $\begin{array}{c}3.422^{* * *} \\
(0.91)\end{array}$ & $\begin{array}{l}1.880 \\
(1.66)\end{array}$ & $\begin{array}{r}-0.079 \\
(2.11)\end{array}$ & $\begin{array}{l}0.680 \\
(1.83)\end{array}$ & 41.21 \\
\hline
\end{tabular}




\section{Table 6: Summary of the Trading Performance After Fee}

This table reports the first quarter (Q1), median, third quarter (Q3), and mean of three measures of the trading performance: annualized total excess return (XR), annualized Sharpe ratio (SR), and maximum drawdown (MDD). Panel (a) contains both the in-sample (upper subpanel) and out-of-sample (lower subpanel) results of the second half of the sample (Jul2008 - Oct2011), Panel (b) contains the full sample (Jan2005 - Oct2011) results. The benchmark risk-free return for XR is based on the U.S. treasury rate. On average it is $5 \%$ for the full sample, which is roughly the return of holding a 60 to 72 months zero coupon government bond issued at the beginning 2005 till its maturity, and 3\% for the second half of the sample, which is roughly the return of holding a 40 months zero coupon government bond issued in Jul 2008 till its maturity.

(a) Second sub-sample (Jul2008 - Oct2011)

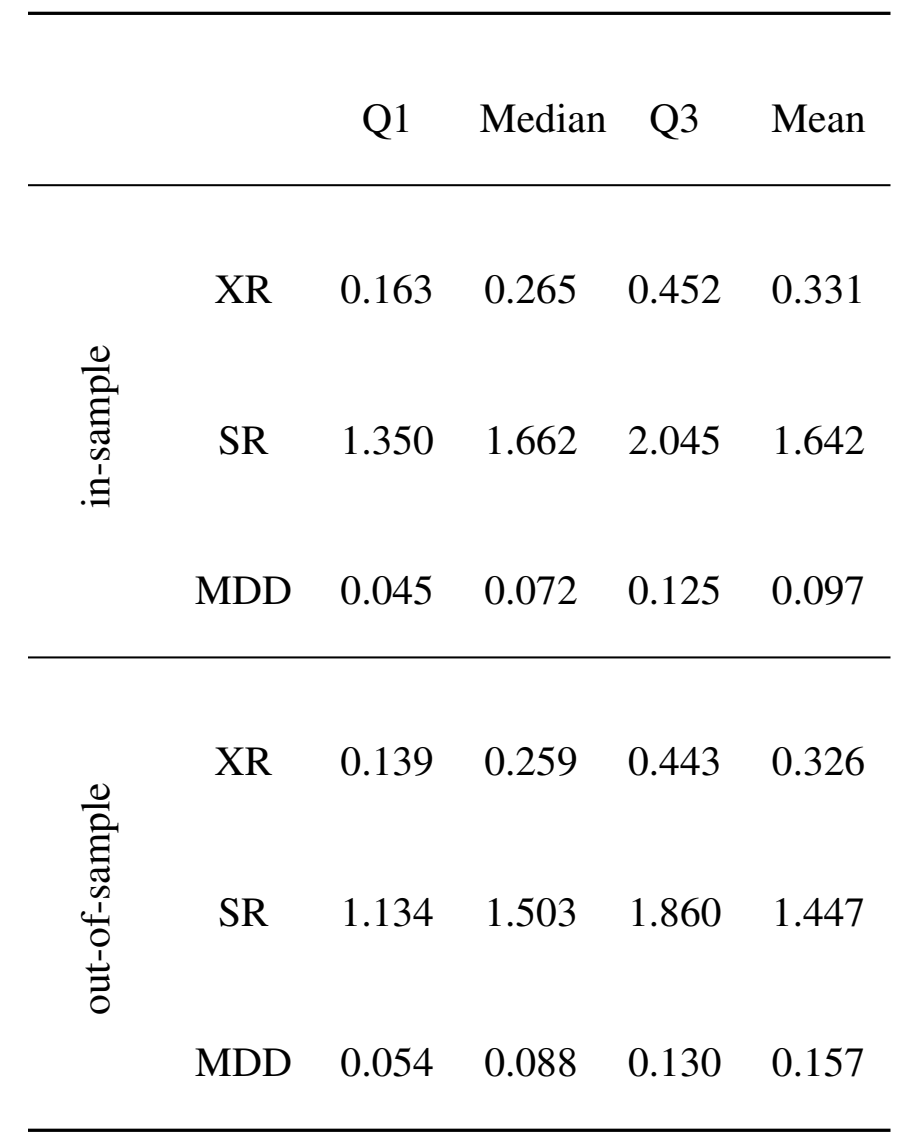

(b) Full sample (Jan2005 - Oct2011)

\begin{tabular}{ccccc}
\hline & Q1 & Median & Q3 & Mean \\
\hline XR & 0.094 & 0.152 & 0.238 & 0.174 \\
& & & & \\
SR & 0.979 & 1.293 & 1.574 & 1.269 \\
& & & & \\
MDD & 0.045 & 0.068 & 0.111 & 0.107 \\
\hline
\end{tabular}


Table 7: Median Annualized Total Excess Return (XR) in Different Ratings and Sectors

This table reports the median of the annualized total excess return (XR) in different ratings and sectors in the second half of the sample (Jul2008 - Oct2011). The benchmark risk-free return for XR is based on the U.S. treasury rate. On average it is $3 \%$ for the second half of the sample, which is roughly the return of holding a 40 months zero coupon government bond issued in Jul 2008 till its maturity. Panel (a) contains the in-sample results, Panel (b) contains the out-of-sample results. The last column (the last row), except the last cell, is the median over each row (column). The last cell at the right-lower conner is the median over all firms.

(a) In-sample

\begin{tabular}{lccccccc}
\hline & AA & A & BBB & BB & B & CCC & Median \\
\hline BM & & -0.020 & 0.243 & 0.466 & 0.418 & 0.322 & 0.322 \\
CG & 0.214 & & 0.239 & 0.375 & 0.773 & 0.544 & 0.375 \\
CS & 0.207 & & 0.327 & 0.562 & 0.867 & 0.480 & 0.480 \\
Fin & 0.247 & & 0.327 & 0.198 & & 0.434 & 0.287 \\
HC & & & 0.123 & 0.389 & 0.297 & 0.798 & 0.343 \\
Ind & 0.137 & & 0.335 & & & 0.812 & 0.335 \\
OG & & 0.171 & 0.254 & 0.352 & & 0.519 & 0.303 \\
Tec & & & 0.175 & 0.078 & 0.547 & 0.746 & 0.361 \\
Tel & 0.307 & & 0.282 & 0.253 & & & 0.282 \\
Uti & 0.205 & 0.304 & 0.260 & & 0.223 & 0.409 & 0.260 \\
Median & 0.210 & 0.171 & 0.257 & 0.363 & 0.483 & 0.519 & 0.265 \\
\hline
\end{tabular}

(b) Out-of-sample

\begin{tabular}{lccccccc}
\hline & AA & A & BBB & BB & B & CCC & Median \\
\hline BM & & 0.327 & 0.231 & 0.442 & 0.464 & 0.310 & 0.327 \\
CG & 0.169 & & 0.233 & 0.540 & 0.575 & 0.430 & 0.430 \\
CS & 0.205 & & 0.323 & 0.420 & 0.634 & 0.398 & 0.398 \\
Fin & 0.221 & & 0.346 & 0.229 & & 0.364 & 0.287 \\
HC & & & 0.122 & 0.466 & 0.245 & 0.967 & 0.355 \\
Ind & 0.126 & & 0.333 & & & 0.691 & 0.333 \\
OG & & 0.129 & 0.250 & 0.367 & & 0.467 & 0.309 \\
Tec & & & 0.131 & 0.032 & 0.416 & 0.285 & 0.208 \\
Tel & 0.188 & & 0.293 & 0.167 & & & 0.188 \\
Uti & 0.179 & 0.232 & 0.254 & & 0.432 & 0.550 & 0.254 \\
Median & 0.183 & 0.232 & 0.252 & 0.393 & 0.448 & 0.430 & 0.259 \\
\hline
\end{tabular}


Table 8: Median Annualized Sharpe Ratio (SR) in Different Ratings and Sectors

This table reports the median of the annualized Sharpe ratio (SR) in different ratings and sectors in the second half of the sample (Jul2008 - Oct2011). Panel (a) contains the in-sample results, Panel (b) contains the out-of-sample results. The last column (the last row), except the last cell, is the median over each row (column). The last cell at the right-lower conner is the median over all firms.

(a) In-sample

\begin{tabular}{lccccccc}
\hline & AA & A & BBB & BB & B & CCC & Median \\
\hline BM & & 0.140 & 1.768 & 1.647 & 2.033 & 1.285 & 1.647 \\
CG & 1.683 & & 1.640 & 1.912 & 2.436 & 2.116 & 1.912 \\
CS & 1.498 & & 1.686 & 1.984 & 1.768 & 1.712 & 1.712 \\
Fin & 1.508 & & 1.702 & 1.659 & & 1.784 & 1.680 \\
HC & & & 1.167 & 2.341 & 1.229 & 2.645 & 1.785 \\
Ind & 1.316 & & 1.590 & & & 1.499 & 1.499 \\
OG & & 1.136 & 1.835 & 1.978 & & 2.660 & 1.906 \\
Tec & & & 1.382 & 1.162 & 1.882 & 2.471 & 1.632 \\
Tel & 1.450 & & 1.561 & 2.387 & & & 1.561 \\
Uti & 1.787 & 1.848 & 1.675 & & 1.446 & 1.932 & 1.787 \\
Median & 1.503 & 1.136 & 1.658 & 1.945 & 1.825 & 1.932 & 1.662 \\
\hline
\end{tabular}

(b) Out-of-sample

\begin{tabular}{lccccccc}
\hline & AA & A & BBB & BB & B & CCC & Median \\
\hline BM & & 1.848 & 1.472 & 1.690 & 1.657 & 1.502 & 1.657 \\
CG & -0.936 & & 1.524 & 1.502 & 2.154 & 1.565 & 1.524 \\
CS & 0.950 & & 1.638 & 1.445 & 1.832 & 1.534 & 1.534 \\
Fin & 1.336 & & 1.664 & 1.576 & & 1.544 & 1.560 \\
HC & & & 1.178 & 1.636 & 1.106 & 2.390 & 1.407 \\
Ind & 1.159 & & 1.467 & & & 1.150 & 1.159 \\
OG & & 1.066 & 1.599 & 1.867 & & 2.034 & 1.733 \\
Tec & & & 0.925 & 0.737 & 1.610 & 1.183 & 1.054 \\
Tel & 1.534 & & 1.491 & 1.541 & & & 1.534 \\
Uti & 1.632 & 1.236 & 1.635 & & 2.049 & 1.708 & 1.635 \\
Median & 1.248 & 1.236 & 1.508 & 1.558 & 1.744 & 1.544 & 1.503 \\
\hline
\end{tabular}


Table 9: Median Maximum Draw-down (MDD) in Different Ratings and Sectors

This table reports the median of the maximum draw-down (MDD) in different ratings and sectors in the second half of the sample (Jul2008 - Oct2011). Panel (a) contains the in-sample results, Panel (b) contains the out-of-sample results. The last column (the last row), except the last cell, is the median over each row (column). The last cell at the right-lower conner is the median over all firms.

(a) In-sample

\begin{tabular}{lccccccc}
\hline & AA & A & BBB & BB & B & CCC & Median \\
\hline BM & & 0.201 & 0.069 & 0.077 & 0.074 & 0.362 & 0.077 \\
CG & 0.036 & & 0.064 & 0.056 & 0.138 & 0.084 & 0.064 \\
CS & 0.085 & & 0.076 & 0.059 & 0.130 & 0.133 & 0.085 \\
Fin & 0.061 & & 0.098 & 0.078 & & 0.084 & 0.081 \\
HC & & & 0.056 & 0.054 & 0.174 & 0.086 & 0.071 \\
Ind & 0.105 & & 0.084 & & & 0.104 & 0.104 \\
OG & & 0.081 & 0.049 & 0.068 & & 0.033 & 0.058 \\
Tec & & & 0.054 & 0.052 & 0.070 & 0.157 & 0.062 \\
Tel & 0.042 & & 0.075 & 0.073 & & & 0.073 \\
Uti & 0.049 & 0.045 & 0.062 & & 0.136 & 0.063 & 0.062 \\
Median & 0.055 & 0.081 & 0.066 & 0.063 & 0.133 & 0.086 & 0.072 \\
\hline
\end{tabular}

(b) Out-of-sample

\begin{tabular}{lccccccc}
\hline & AA & A & BBB & BB & B & CCC & Median \\
\hline BM & & 0.153 & 0.099 & 0.159 & 0.073 & 0.261 & 0.153 \\
CG & 0.025 & & 0.081 & 0.099 & 0.112 & 0.106 & 0.099 \\
CS & 0.081 & & 0.080 & 0.137 & 0.109 & 0.108 & 0.108 \\
Fin & 0.074 & & 0.102 & 0.060 & & 0.114 & 0.088 \\
HC & & & 0.073 & 0.102 & 0.187 & 0.073 & 0.088 \\
Ind & 0.131 & & 0.094 & & & 0.106 & 0.106 \\
OG & & 0.084 & 0.073 & 0.059 & & 0.124 & 0.078 \\
Tec & & & 0.087 & 0.063 & 0.118 & 0.243 & 0.103 \\
Tel & 0.056 & & 0.080 & 0.082 & & & 0.080 \\
Uti & 0.074 & 0.084 & 0.083 & & 0.023 & 0.085 & 0.083 \\
Median & 0.074 & 0.084 & 0.082 & 0.090 & 0.110 & 0.108 & 0.088 \\
\hline
\end{tabular}


Figure 1: Aggregate returns: histograms and cumulative path

The first (second) panel shows the histogram of the aggregate weekly returns from July 2008 to October 2011 (July 2009 to October 2011 ). The third panel shows the cumulative returns over time. All returns are weekly and the unit of x-axes in the first and second panel, and y-axis in the third panel is percent.
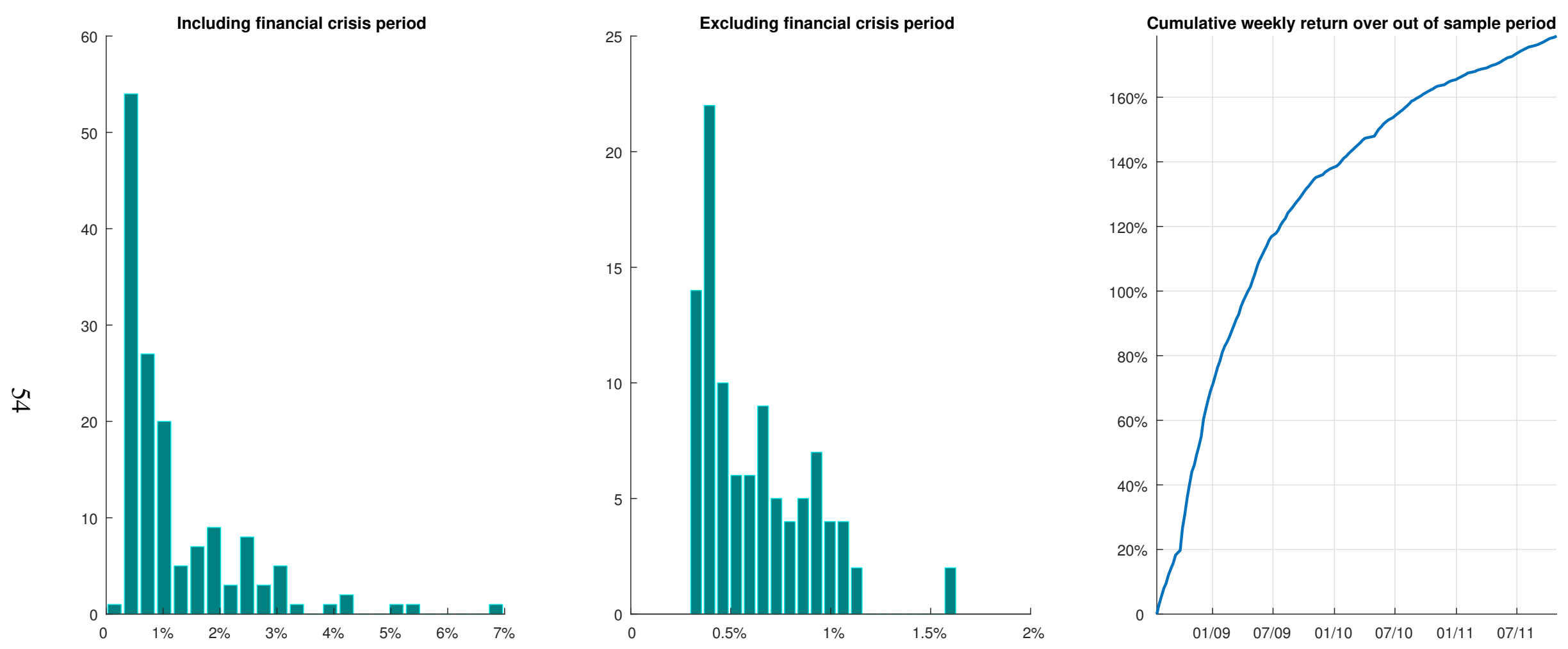
Figure 2: Summary of the Performance Fee Estimates

This figure shows the estimates of the weekly performance fee $\Delta$ with $\gamma=1$ (left panel) and $\gamma=10$ (right panel) when the target volatility varies from $3 \%, 4 \%, \ldots$, to $8 \%$. The aggregate out-of-sample returns of the trading strategy are compared to the returns (before expenses) of the three benchmarks ETF funds: LQD, SPY, and VXX.

$$
\text { (a) } \gamma=1
$$

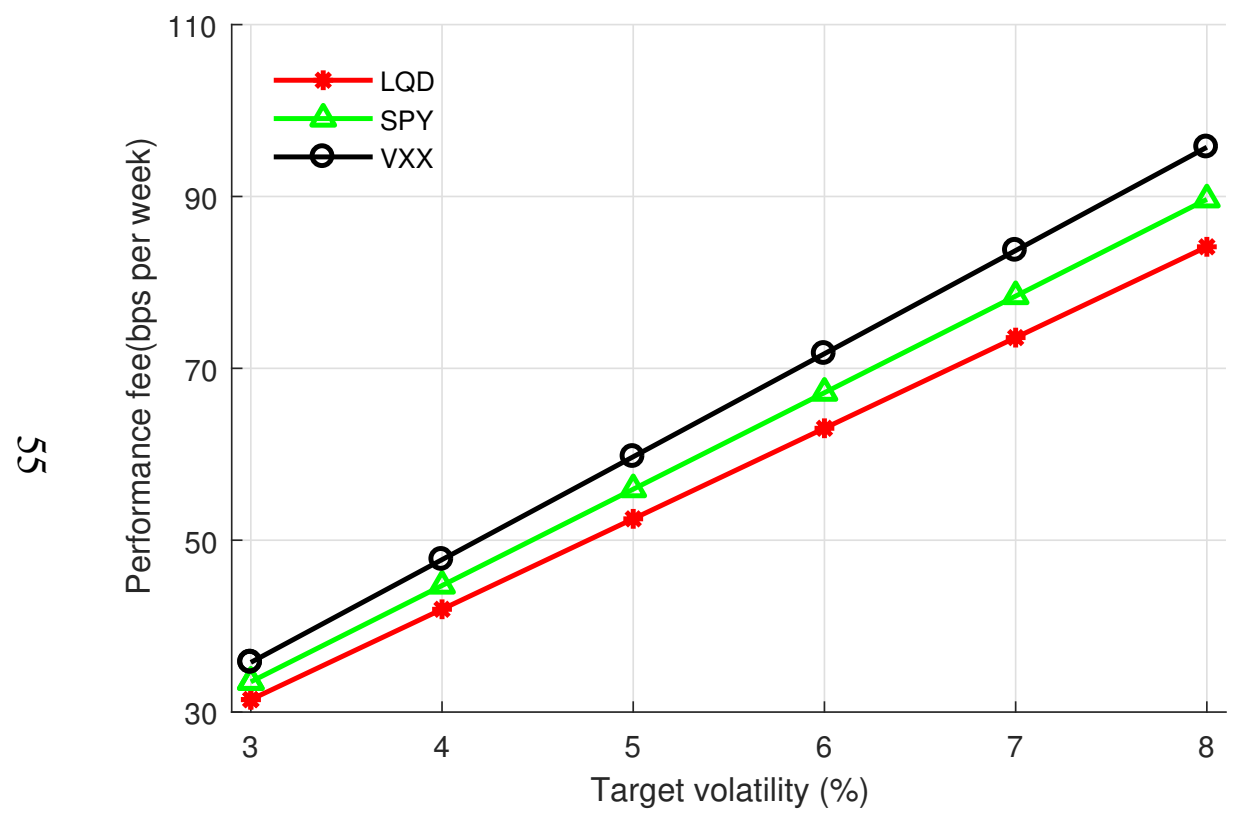

(b) $\gamma=10$

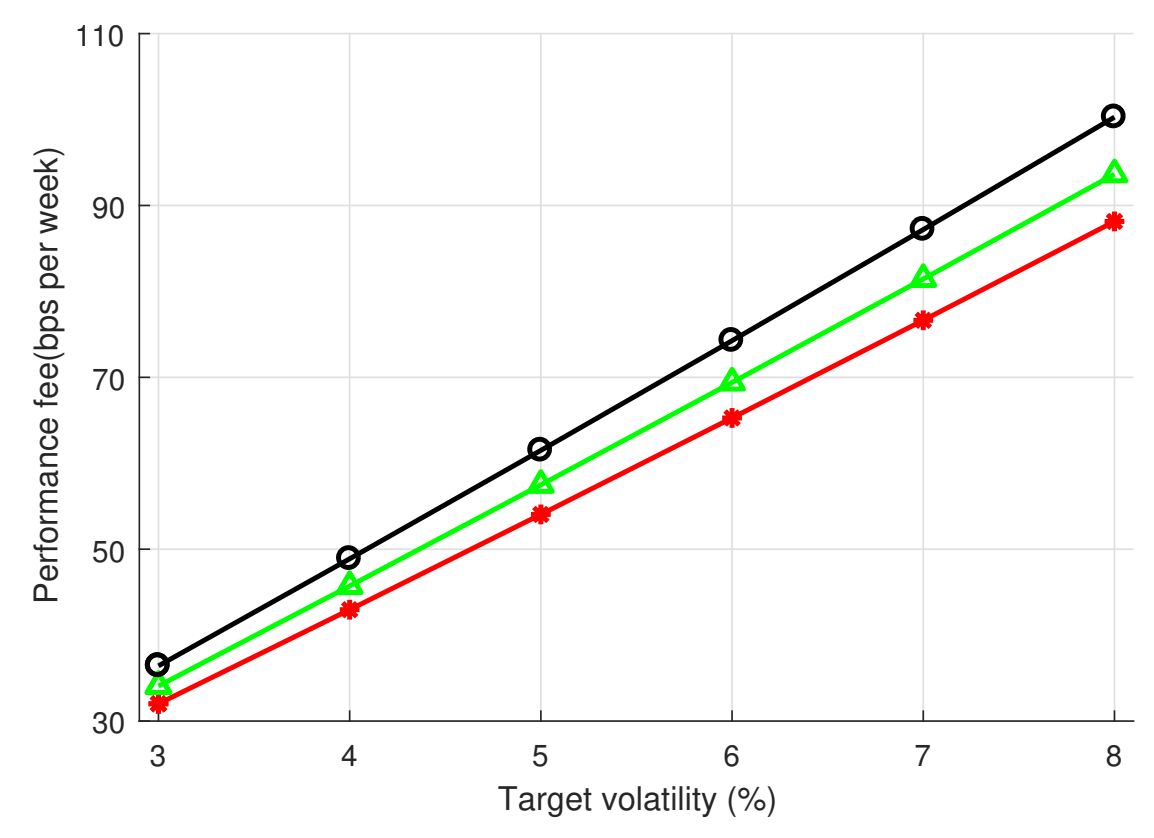


Table A1: Results of the return factor regressions using the GFI data

This table reports the OLS coefficients of the return factor regressions. Newey-West standard errors are in brackets. $* * *, * *, *$ represent the significance levels at $1 \%, 5 \%$, and $10 \%$, respectively. The LHS variable is the aggregate returns of the trading strategy from in-sample and out-of-sample. Besides the control variables, USCDS, NFCI, and MES, the RHS variables include CRDTSPRD and NOISE for regression (1); CRDTSPRD, CRDTSPRD ${ }^{2}$, NOISE, and NOISE ${ }^{2}$ for regression (2); CRDTSPRD ${ }^{2}$ and NOISE$^{2}$ for regression (3). The upper panel reports full sample (in-sample Jan 2005 - Oct 2011) results, the middle panel reports in-sample (Jul 2008 - Oct 2011) results, and the lower panel reports out-of-sample (Jul 2008 - Oct 2011) results. To make the coefficients comparable in magnitude, CRDTSPRD ${ }^{2}$ and NOISE ${ }^{2}$ are multiplied by 1000 and 100,000 in the regressions.

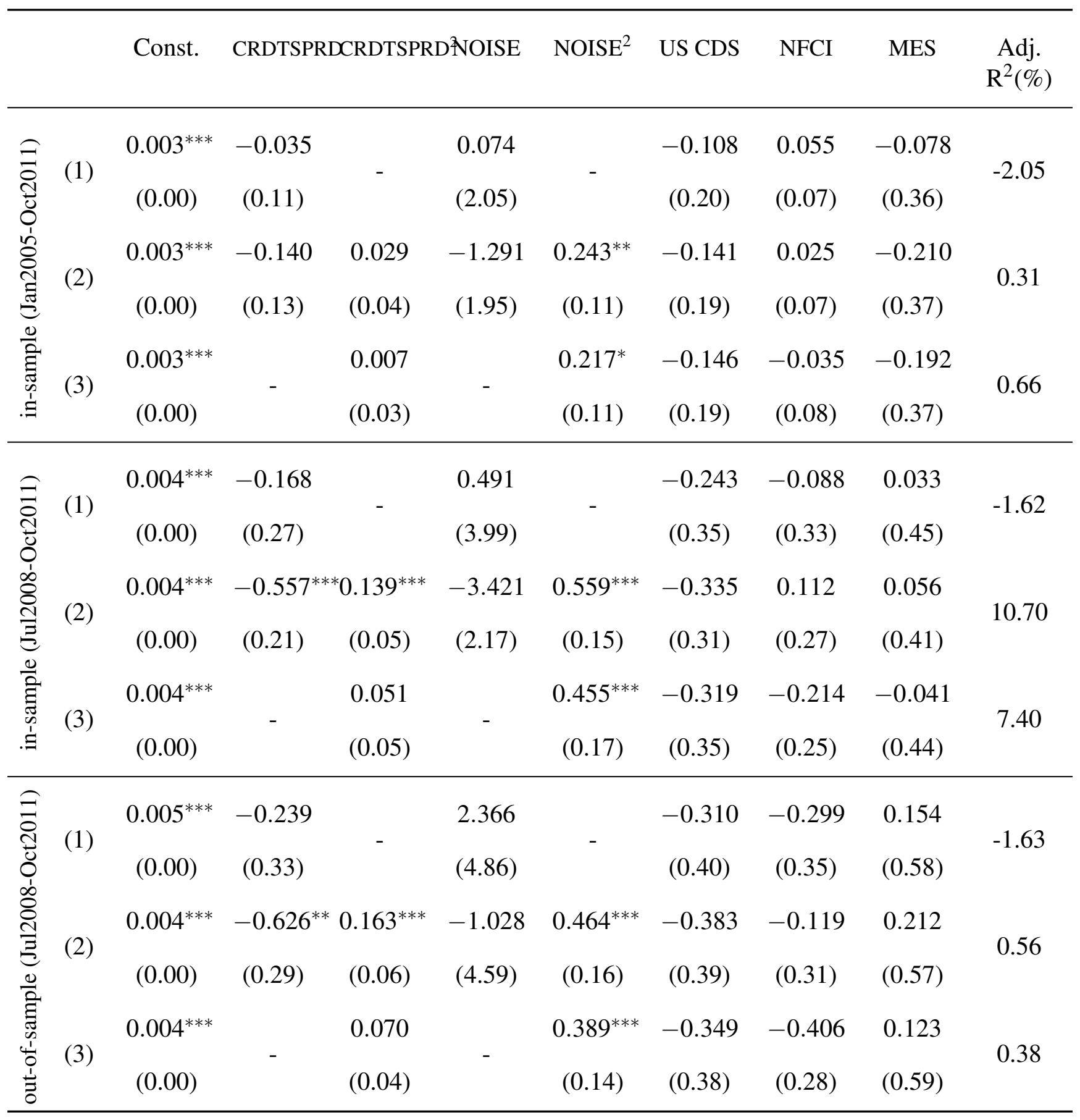


Figure A1: Aggregate returns: histograms and cumulative path GFI data

The first (second) panel shows the histogram of the aggregate weekly returns from July 2008 to October 2011 (July 2009 to October 2011). The third panel shows the cumulative returns over time. All returns are weekly and the unit of $\mathrm{x}$-axes in the first and second panel, and y-axis in the third panel is percent.
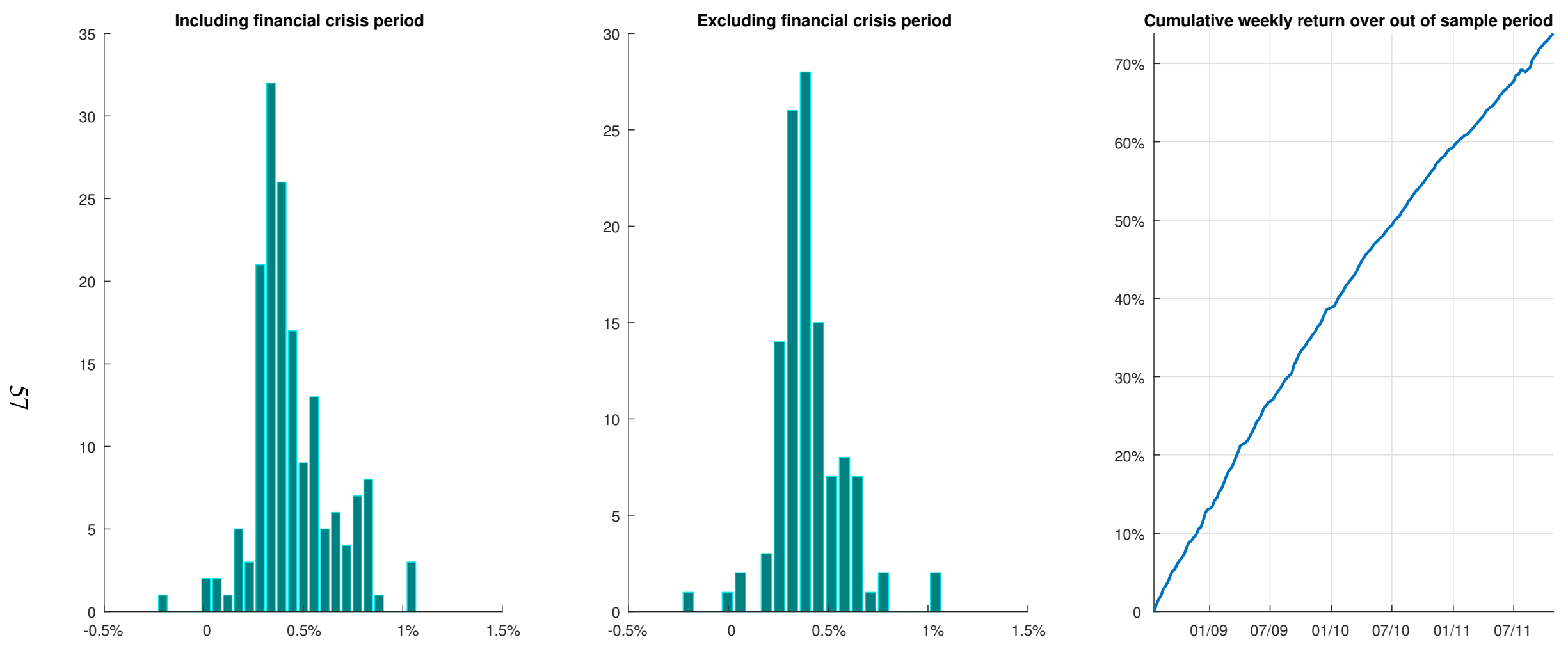


\title{
Online Appendices to
}

\section{Exploring Mispricing in the Term Structure of CDS Spreads}

\author{
Robert Jarrow, Haitao Li, Xiaoxia Ye, and May Hu*
}

April 2018

\section{Tables of summary descriptives of Markit data}

To save the space in the main text, tables of summary descriptives of Markit data are presented in this section. Table 1 provides a summary of the distribution of the 500 firms by ratings and sectors. Tables 2 and 3 provide the mean and standard deviation of CDS spreads for different ratings and sectors.

\section{Identification of the Recovery Rate}

In this appendix, we follow Pan and Singleton (2008)'s procedure to examine the CDS upfront sensitivity to the loss rate $y$, and then conduct a Monte-Carlo simulation exercise regarding the accuracy of model estimation. The first panel (left and upper) in Fig. 1 shows the sensitivity of the CDS upfronts at four maturities (two, five, 10, and 20 years) to variations in $y$ while holding $y Z$ fixed at 52 bps. When computing the CDS upfronts, we use the following parameter values: $\alpha=0.002, \kappa=-0.189$, $\sigma=0.143, c_{0}=0, \alpha^{P}=0.017, \kappa^{P}=0.914$, and $y=0.428$. These values are the median parameter estimates in the main text. We can see that the longer maturity the CDS upfronts depend the more on $y$.

*Jarrow (email: raj15@cornell.edu) is at the Johnson Graduate School of Management, Cornell University, Ithaca, NY 14850 and Kamakura Corporation, Honolulu, Hawaii 96815. Li (email: htli@ckgsb.edu.cn) is at the Cheung Kong Graduate School of Business, Beijing, China 100738. Ye (email: x.ye4@bradford.ac.uk) is at School of Management, University of Bradford and Management School, University of Liverpool, UK, L69 7ZH. Hu (email: may.hu@ deakin.edu.au) is at Deakin University, Australia, VIC 3125. 
We present the partial derivative of the CDS upfronts with respect to $y$ in other panels (from the second to the end) of Fig. 1. To take into account the effect of fixing $y Z$ in the partial derivative, we consider a variant of the original upfront pricing formula defined as:

$$
U^{\prime}(x, \tau ; y)=U\left(\frac{x}{y}, \tau ; y\right)
$$

where $U$ is given in (5). The partial derivatives in Fig. 1 are calculated as $\partial U^{\prime} / \partial y$. These figures show non-zero partial derivatives over a wide range of values for five parameters, $\alpha, \kappa, \sigma, y Z$, and $y$, suggesting that the loss rate $y$ can be identified separately from $Z$.

To assess the unbiasedness of the estimates, we perform a Monte-Carlo simulation exercise. Based on the true parameter values of $\alpha^{P}, \kappa^{P}$, and $\sigma$ in Table 4, four years of weekly $Z$ 's (matching the dates in our full sample period) are simulated. Given the other true parameters in Table 4, the simulated Z's, and the corresponding risk-free discount factors, the nine CDS upfronts with maturities of one, two, three, five, seven, 10, 15, 20, and 30 years are computed at every point in time. Here we consider two true values for $y$ : the median value of $y$ in the summary of parameter estimates in the main text and $75 \%$ as in Pan and Singleton (2008). We then add measurement noise consistent with the estimated level to these CDS upfronts and estimate the model parameters using the UKF procedure. In total, we perform this simulation and estimation 500 times. The results are summarized in Table 4.

We find that while $\alpha, \kappa, \sigma, c_{0}$, and $\alpha^{P} / \kappa^{P}$ can be estimated with high precision, there exist biases in the estimates of $\kappa^{P}$ and $y$. The bias in $\kappa^{P}$ is somewhat expected given the well documented difficulty in estimating the mean reversion parameter under the $P$ measure. ${ }^{1}$ The downward bias in the estimate of $y$ is more severe when the true value of $y$ is smaller. The bias in $\kappa^{P}$ has little effect on accurately identifying $\alpha, \kappa, \sigma$, and $c_{0}$ in the model, and is never used in the development of the arbitrage trading strategy, therefore a bias correction in $\kappa^{P}$ is not necessary in the current paper. However, $1-y$ represents the recovery rate implicit in the CDS upfronts, it seems necessary to develop a correction based on Table 4 for the sake of judiciously interpreting the recovery rate results presented in the main text. When the true $y=0.428(y=0.75)$, the biased estimate $\hat{y}=0.3(\hat{y}=0.68)$, and downward bias is $0.13(0.07)$. We

\footnotetext{
${ }^{1}$ For example, see Duffee and Stanton (2012) and Yu (2012).
} 
linearly interpolate the biases. Therefore the correction formulae for the recovery rate are given by

$$
\begin{aligned}
r e c^{\text {corrected }} & =1-y^{\text {corrected }}=1-[\hat{y}+\operatorname{correction}(\hat{y})], \\
\operatorname{correction}(y) & =0.07+\frac{0.13-0.07}{0.3-0.68}(y-0.68)=0.07-0.16(y-0.68) .
\end{aligned}
$$

Since the development of the trading strategy does not require an unbiased estimate of $y$, we do not apply this correction to the estimates used in the main text.

\section{Weight calculation in a real-life example}

In this section, we illustrate the portfolio weight calculation using data of a randomly selected firm "AMR group" on a randomly selected date "27-May-2005". First, given the estimated model, $H_{1}$ and $H_{2}$ at the nine maturities

$$
\vec{\tau}=[1,2,3,5,7,10,15,20,30]^{\top}
$$

can be easily calculated:

$$
\begin{aligned}
& H_{1}=[0.48,0.65,0.63,0.51,0.44,0.38,0.32,0.30,0.28]^{\top} \\
& H_{2}=[-0.54,-1.50,-2.07,-2.15,-1.92,-1.63,-1.36,-1.24,-1.15]^{\top} .
\end{aligned}
$$

Also, we have

$$
\mathbb{E}_{t}\left(\Delta \varepsilon_{t+\Delta t}^{\vec{\tau}}\right)=[0.004,0.004,0.001,0.000,0.011,0.002,-0.005,0.003,0.003]^{\top}
$$


Second, following the procedure of (22) and (23) in the main text, we can get

$$
\left[\vec{M}\left(Z_{t}, \vec{\tau}^{1}\right), \cdots, \vec{M}\left(Z_{t}, \vec{\tau}^{9}\right)\right]=\left[\begin{array}{ccccccccc}
1.00 & -0.61 & 0.00 & 0.00 & 0.00 & 0.00 & 0.00 & 0.00 & 0.00 \\
-1.64 & 1.00 & -0.48 & 0.00 & 0.00 & 0.00 & 0.00 & 0.00 & 0.00 \\
0.93 & -0.57 & 1.00 & -0.14 & 0.00 & 0.00 & 0.00 & 0.00 & 0.00 \\
0.00 & 0.00 & -0.63 & 1.00 & 0.25 & 0.00 & 0.00 & 0.00 & 0.00 \\
0.00 & 0.00 & 0.00 & -0.97 & 1.00 & -0.64 & 0.00 & 0.00 & 0.00 \\
0.00 & 0.00 & 0.00 & 0.00 & -1.50 & 1.00 & -0.32 & 0.00 & 0.00 \\
0.00 & 0.00 & 0.00 & 0.00 & 0.00 & -0.29 & 1.00 & -0.40 & 0.66 \\
0.00 & 0.00 & 0.00 & 0.00 & 0.00 & 0.00 & -0.67 & 1.00 & -1.66 \\
0.00 & 0.00 & 0.00 & 0.00 & 0.00 & 0.00 & 0.00 & -0.60 & 1.00
\end{array}\right] .
$$

Next, since we can either long or short each column in $\left[\vec{M}\left(Z_{t}, \vec{\tau}^{1}\right), \cdots, \vec{M}\left(Z_{t}, \vec{\tau}^{9}\right)\right]$, we have $\sum_{i=1}^{9} \frac{9 !}{i !(9-i) !}=$ 511 different weight vector $W\left(Z_{t}, I_{t}, \vec{\tau}\right)$. For example, if we short the first column and long all others,

$$
\begin{aligned}
W\left(Z_{t}, I_{t}, \vec{\tau}\right)= & {\left[\begin{array}{ccccccccc}
-1.00 & -0.61 & 0.00 & 0.00 & 0.00 & 0.00 & 0.00 & 0.00 & 0.00 \\
1.64 & 1.00 & -0.48 & 0.00 & 0.00 & 0.00 & 0.00 & 0.00 & 0.00 \\
-0.93 & -0.57 & 1.00 & -0.14 & 0.00 & 0.00 & 0.00 & 0.00 & 0.00 \\
0.00 & 0.00 & -0.63 & 1.00 & 0.25 & 0.00 & 0.00 & 0.00 & 0.00 \\
0.00 & 0.00 & 0.00 & -0.97 & 1.00 & -0.64 & 0.00 & 0.00 & 0.00 \\
0.00 & 0.00 & 0.00 & 0.00 & -1.50 & 1.00 & -0.32 & 0.00 & 0.00 \\
0.00 & 0.00 & 0.00 & 0.00 & 0.00 & -0.29 & 1.00 & -0.40 & 0.66 \\
0.00 & 0.00 & 0.00 & 0.00 & 0.00 & 0.00 & -0.67 & 1.00 & -1.66 \\
0.00 & 0.00 & 0.00 & 0.00 & 0.00 & 0.00 & 0.00 & -0.60 & 1.00
\end{array}\right] \times\left[\begin{array}{l}
1 \\
1 \\
1 \\
1 \\
1 \\
1 \\
1 \\
1 \\
1 \\
1 \\
1 \\
1
\end{array}\right] }
\end{aligned}
$$

And the expected return of this portfolio is

$$
W\left(Z_{t}, I_{t}, \vec{\tau}\right)^{\top} \mathbb{E}_{t}\left(\Delta \varepsilon_{t+\Delta t}^{\vec{\tau}}\right)=-0.01
$$


Following this procedure, we will have 511 different portfolios with different expected returns. We choose the weight associated with the largest return, provided it is positive, as the target weight in that week. If the largest return is negative, we set the target weight to zero. In this real-life example, the largest return among the 511 return candidates is 0.042 and its associated weight is

$$
[-1.61,3.12,-2.37,-0.12,2.62,-2.18,-1.77,3.34,-1.60]^{\top}
$$

\section{Results with $\Delta t=$ one month}

To test the robustness of the results using holding periods other than one week, we set $\Delta t=$ one month and redo the analysis in Section 6.1 of the main text. The graphical results are in Fig. 2. The annualized return is $141.2 \%$ and annualized Sharpe ratio is 2.6. When the financial crisis period is excluded, the annualized return is $104.8 \%$ and the annualized Sharpe ratio is 3.1. Apparently, when $\Delta t=$ one month, the returns are much higher, therefore the evidence of mispricing is robust to the choice of holding period $\Delta t$. At the same time, we do see much lower Sharpe ratios, this means with $\Delta t=$ one month, the return volatility is also higher.

\section{Results using Overnight Index Swaps (OIS) and Interest Rate Swaps (IRS) as discount rates}

To test the robustness of the results using alternative discount rates, we bootstrap OIS and IRS to get zero yields and use them as discount rates and benchmark rates. The OIS and IRS data are obtained from Datastream. The CDS premia are converted to upfronts using the zero yields bootstrapped from the OIS and IRS. The Sharpe ratios are calculated using the zero yields as the "risk free" benchmark. The annualized return is $58.4 \%$ and annualized Sharpe ratio is 9.1 . When the financial crisis period is excluded, the annualized return is $31 \%$ and the annualized Sharpe ratio is 15.6. From Fig. 3 we can see the results have no essential difference than those in the main text. 


\section{Results when financial firms are excluded}

This section presents the results without financial firms. The annualized return is $62.8 \%$ and annualized Sharpe ratio is 7.7. When the financial crisis period is excluded as well, the annualized return is $33.6 \%$ and the annualized Sharpe ratio is 16.6. From Fig. 4 we can see the results have no essential difference than those in the main text.

\section{Alternative bid-ask term structure assumption}

In this section, we show the summary results of the performance after fee when using an alternative specification for $\delta_{i}$,

$$
\delta_{i}= \begin{cases}14 \mathrm{bps} & \text { for } i=5 y r \\ 2 \delta_{5 y r} & \text { for } i=1,2,3,7,15,20, \text { and } 30 y r \\ 1.5 \delta_{5 y r} & \text { for } i=10 y r\end{cases}
$$

With this alternative specification of bid-ask spreads term structure which are overall higher than those in the main text, in Table 5 we only see slightly different (slightly worse but expected) results than those in the main text. These results show our conclusion is robust to the specification.

\section{Two-factor CDS upfront model}

Here we develop a simple two-factor model which uses Nelson Siegel (NS) parametrization (see Nelson and Siegel, 1987; Diebold and Li, 2006) to fit the GFI curve data. As shown in the main text, the pricing formula of the upfronts at $t$ for a protection between $t$ and $t+\tau$ is given by

$$
U\left(Z_{1, t}, Z_{2, t} ; \tau\right)=\frac{y \sum_{i=1}^{4 \tau} P\left(t, t+\frac{2 i-1}{8}\right) \mathbb{E}_{2}\left(t, t+\frac{2 i-1}{8}\right)-C\left\{\sum_{i=1}^{4 \tau} P\left(t, t+\frac{i}{4}\right) \mathbb{E}_{1}\left(t, t+\frac{i}{4}\right)+\frac{1}{8} \sum_{i=1}^{4 \tau} P\left(t, t+\frac{2 i-1}{8}\right) \mathbb{E}_{2}\left(t, t+\frac{2 i-1}{8}\right)\right\}}{4} .
$$


where $\mathbb{E}_{1}$ has an exponential affine form and $\mathbb{E}_{2}$ is $\mathbb{E}_{1}$ times an affine form. Here we specify the affine formulation as NS curve,

$$
\begin{aligned}
& \mathbb{E}_{1}(t, T)=\exp \left\{\beta_{1}\left[\frac{\left(1-e^{-\lambda_{1}(T-t)}\right)}{\lambda_{1}(T-t)}-e^{-\lambda_{1}(T-t)}\right]+Z_{1, t}+\frac{\left(1-e^{-\lambda_{1}(T-t)}\right)}{\lambda_{1}(T-t)} Z_{2, t}\right\}, \\
& \mathbb{E}_{2}(t, T)=\mathbb{E}_{1}(t, T)\left\{\beta_{2}\left[\frac{\left(1-e^{-\lambda_{2}(T-t)}\right)}{\lambda_{2}(T-t)}-e^{-\lambda_{2}(T-t)}\right]+Z_{1, t}+\frac{\left(1-e^{-\lambda_{2}(T-t)}\right)}{\lambda_{2}(T-t)} Z_{2, t}\right\} .
\end{aligned}
$$

In the calibration, $y$ is set to $60 \%$. The free parameters in the two-factor model are $\beta_{1}, \beta_{2}, \lambda_{1}$, and $\lambda_{2}$. Similar to Ang and Longstaff (2013), the parameters are calibrated by a nonlinear least square. Intuitively, the algorithm chooses first a trial set of the parameters $\beta_{1}, \beta_{2}, \lambda_{1}$, and $\lambda_{2}$. Given these parameters, the model upfronts at time $t$ depend only on the value of $Z_{1, t}$ and $Z_{2, t}$, which we can calibrate by a nonlinear least squares fit of the model to the all upfronts at time $t$. Basically, the value of $Z_{1, t}$ captures the level of the corresponding CDS curve while the $Z_{2, t}$ captures the slope.

\section{Strategy using the two-factor model}

Given the two-factor upfront model, we consider the second-order expansion of the upfronts pricing function $U\left(Z_{1, t}, Z_{2, t} ; \tau\right)$ around the state variables $Z_{1, t}$ and $Z_{2, t}$ with the following first and second order derivatives $J_{1}\left(Z_{1}, Z_{2} ; \tau\right)=\frac{\partial U\left(Z_{1}, Z_{2} ; \tau\right)}{\partial Z_{1}}, J_{2}\left(Z_{1}, Z_{2} ; \tau\right)=\frac{\partial U\left(Z_{1}, Z_{2} ; \tau\right)}{\partial Z_{2}}, H_{11}\left(Z_{1}, Z_{2} ; \tau\right)=\frac{\partial^{2} U\left(Z_{1}, Z_{2} ; \tau\right)}{\partial Z_{1}^{2}}$, $H_{12}\left(Z_{1}, Z_{2} ; \tau\right)=\frac{\partial^{2} U\left(Z_{1}, Z_{2} ; \tau\right)}{\partial Z_{1} \partial Z_{2}}$, and $H_{22}\left(Z_{1}, Z_{2} ; \tau\right)=\frac{\partial^{2} U\left(Z_{1}, Z_{2} ; \tau\right)}{\partial Z_{2}^{2}}$. Similar to the main text, we have

$$
\begin{aligned}
U\left(Z_{1, t+\Delta t}, Z_{2, t+\Delta t} ; \tau-\Delta t\right) & \approx U\left(Z_{1, t}, Z_{2, t} ; \tau\right)+J_{1}\left(Z_{1, t}, Z_{2, t} ; \tau\right)\left(Z_{1, t+\Delta t}-Z_{1, t}\right)+J_{2}\left(Z_{1, t}, Z_{2, t} ; \tau\right)\left(Z_{2, t+\Delta t}-Z_{2, t}\right) \\
& +\frac{1}{2} H_{11}\left(Z_{1, t}, Z_{2, t} ; \tau\right)\left(Z_{1, t+\Delta t}-Z_{1, t}\right)^{2}+\frac{1}{2} H_{22}\left(Z_{1, t}, Z_{2, t} ; \tau\right)\left(Z_{2, t+\Delta t}-Z_{2, t}\right)^{2} \\
& +H_{12}\left(Z_{1, t}, Z_{2, t} ; \tau\right)\left(Z_{1, t+\Delta t}-Z_{1, t}\right)\left(Z_{2, t+\Delta t}-Z_{2, t}\right)
\end{aligned}
$$

Denote any maturity in $\left(\tau=1,2,3,4,5,7\right.$, and 10) by $\tau_{0}$, and the five most adjacent maturities of $\tau_{0}$ by $\tau_{1}$ to $\tau_{5}$. To see how $\tau_{1}$ and $\tau_{5}$ are chosen, let us look at a few examples: if $\tau_{0}=1$, then $\tau_{1}=2, \tau_{2}=3$, 
$\tau_{3}=4, \tau_{4}=5$, and $\tau_{5}=7$; if $\tau_{0}=5$, then $\tau_{1}=2, \tau_{2}=3, \tau_{3}=4, \tau_{4}=7$, and $\tau_{5}=10$; and if $\tau_{0}=10$, then $\tau_{1}=2, \tau_{2}=3, \tau_{3}=4, \tau_{4}=5$, and $\tau_{5}=7$. Therefore, given $\Delta t$, at time $t$ for a unit notional amount of CDS contract with maturity of $\tau_{0}$, we employ five other CDS contracts with maturities of $\tau_{1}$ to $\tau_{5}$ to form a hedged portfolio, whose value is immune to the variation of $Z_{1, t}$ and $Z_{2, t}$ up to the second order but subject to the variation of the pricing residuals.

Denote the portfolio weight by $\underbrace{M\left(Z_{1, t}, Z_{2, t}, \vec{\tau}^{i}\right)}_{6 \times 1}$, where $\vec{\tau}^{i}=\left[\tau_{0}, \tau_{1}, \cdots, \tau_{5}\right]^{\top}$. We fix the first element in $M\left(Z_{1, t}, Z_{2, t}, \overrightarrow{\tau^{i}}\right)$ at one for any $\vec{\tau}^{i}$. So the future value of the portfolio at time $t+\Delta t$ is given by

$$
M\left(Z_{1, t}, Z_{2, t}, \vec{\tau}^{i}\right)^{\top} \operatorname{Upfronts}_{t+\Delta t}^{\overrightarrow{\tau^{i}-\Delta t}}=M\left(Z_{1, t}, Z_{2, t}, \vec{\tau}^{i}\right)^{\top} U\left(Z_{t+\Delta t}, \vec{\tau}^{i}-\Delta t\right)+M\left(Z_{1, t}, Z_{2, t}, \vec{\tau}^{i}\right)^{\top} \varepsilon_{t+\Delta t}^{\vec{\tau}^{i}-\Delta t}
$$

Now if we specify the second to fifth elements in $M\left(Z_{1, t}, Z_{2, t}, \vec{\tau}^{i}\right)$ such that

$$
M^{\top}\left[J_{1}, J_{2}, H_{11}, H_{12}, H_{22}\right]=\underbrace{0}_{1 \times 6}
$$

then we have the hedged portfolio. That is

$$
M\left(Z_{1, t}, Z_{2, t}, \overrightarrow{\tau^{i}}\right)^{\top} \operatorname{Upfronts}_{t+\Delta t} \overrightarrow{\vec{\tau}^{i}-\Delta t} \approx M\left(Z_{1, t}, Z_{2, t}, \overrightarrow{\tau^{i}}\right)^{\top} \operatorname{Upfronts}_{t} \vec{\tau}^{i}+M\left(Z_{1, t}, Z_{2, t}, \vec{\tau}^{i}\right)^{\top} \Delta \varepsilon_{t+\Delta t} \cdot \overrightarrow{\vec{\tau}^{i}}
$$

The remaining procedure is the same as that in the main text, except that $I_{t}$ can be now chosen from $\sum_{i=1}^{7} \frac{7 !}{i !(7-i) !}=127$ different vectors of -1 and 1 combinations. In the implementation, given $\beta_{1}, \beta_{2}, \lambda_{1}$, $\lambda_{2}$ and $Z_{1, t}, Z_{2, t},\left[J_{1}, J_{2}, H_{11}, H_{12}, H_{22}\right]$ can be calculated numerically.

\section{The strategy with transaction costs}

For a trade, the primary transaction cost is the effective bid-ask full-spreads (double the effective bid-ask half-spreads), i.e., the capital gain after transaction costs $\left(C G^{a}\right)$ is given by

$$
C G_{t+\Delta t}^{a}=C G_{t+\Delta t}^{b}-\left|N A_{t} I_{t}-N A_{t-\Delta t} I_{t-\Delta t}\right| \delta
$$


where $\delta$ is the effective bid-ask half-spread and $N A_{t-\Delta t} I_{t-\Delta t}$ is the position from previous holding at $t-\Delta t$

The secondary transaction cost is collateral requirements. Commonly known collateral requirements are posted to control for the counterparty risk, i.e., the collateral that is put down for the sake of clearing safely CDS positions of the major derivatives dealers. Heller and Vause (2012) show that in average the initial margins (IM) relative to the total notional is about $3 \% .^{2}$ In addition to the IM, the variation margins are also common in CDS trading due to the mark-to-market nature of the CDS contracts. However, the variation margins are much smaller relatively to the IM.

Of course, there are also implicit collateral requirements for shorting CDS contracts (selling protections) to deal with the credit event risk of the underlying assets. ${ }^{3}$ These collateral requirements should be on top of those IM for the counterparty risk. To our knowledge, there are no formal studies on these collateral requirements. This is probably because the major derivatives dealers are believed to have the capital buffer to cover the loss due to the defaults of the underlying assets, which are relatively rare in normal times.

Given these transaction costs, the return to a trade after transaction costs, assuming the requirements of the variation margins are satisfied during the whole duration of the trade, is given by

$$
r_{t}^{a}=\frac{C G_{t}^{a}}{I M_{t-\Delta t}} .
$$

Notice that here $r_{t}^{a}$ is a return before the interest paid to keep $I M_{t-\Delta t}$ in the account. In the later section, we will take the risk-free interest rate into consideration to calculate annualized excess returns and Sharpe ratios.

\footnotetext{
${ }^{2}$ It is worth noting that we do not use $3 \%$ as the ratio of IM to total notional in our empirical study. We instead assume a much higher IM to keep our results as conservative as possible. See the main text for details.

${ }^{3}$ In the case of selling credit risk protections, the seller should be able to deliver the protection (total notional times the rate of loss given default) when the underlying asset defaults. So certain amount of collateral from the seller is requested to ease the concerns of the buyer that the seller might also default when the underlying asset defaults, even though it is relatively rare in normal times.
} 
Therefore the expected capital gain after transaction costs at time $t$ is revised to be

$$
\begin{aligned}
\mathbb{E}_{t}\left(C G_{t+\Delta t}^{a}\right) & =W\left(Z_{t}, I_{t}, \vec{\tau}\right)^{\top} \mathbb{E}_{t}\left(\text { Upfronts }_{t+\Delta t}^{\vec{\tau}-\Delta t}-\text { Upfronts }_{t}^{\vec{\tau}}\right)-\left|W\left(Z_{t}, I_{t}, \vec{\tau}\right)^{\top}-\tilde{W}_{t-\Delta t}^{\top}\right| \vec{\delta} \\
& \approx W\left(Z_{t}, I_{t}, \vec{\tau}\right)^{\top} \mathbb{E}_{t}\left(\Delta \varepsilon_{t+\Delta t}^{\vec{\tau}}\right)-\left|W\left(Z_{t}, I_{t}, \vec{\tau}\right)^{\top}-\tilde{W}_{t-\Delta t}^{\top}\right| \vec{\delta}
\end{aligned}
$$

At each point in time, $t$, the optimization problem for the portfolio weight $W\left(Z_{t}, I_{t}, \vec{\tau}\right)$ is revised to take into consideration of the bid-ask spreads:

$$
\tilde{W}_{t}=\left\{\begin{array}{cc}
W\left(Z_{t}, \tilde{I}_{t}, \vec{\tau}\right) & \text { if } W\left(Z_{t}, \tilde{I}_{t}, \vec{\tau}\right)^{\top} \mathbb{E}_{t}\left(\Delta \varepsilon_{t+\Delta t}^{\vec{\tau}}\right)>\left|W\left(Z_{t}, I_{t}, \vec{\tau}\right)^{\top}-\tilde{W}_{t-\Delta t}^{\top}\right| \vec{\delta} \\
0_{9 \times 1} & \text { if } W\left(Z_{t}, \tilde{I}_{t}, \vec{\tau}\right)^{\top} \mathbb{E}_{t}\left(\Delta \varepsilon_{t+\Delta t}^{\vec{\tau}}\right) \leqslant\left|W\left(Z_{t}, I_{t}, \vec{\tau}\right)^{\top}-\tilde{W}_{t-\Delta t}^{\top}\right| \vec{\delta}
\end{array},\right.
$$

where $^{4}$

$$
\tilde{I}_{t}=\arg \max _{I_{t} \in \mathscr{I}}\left\{W\left(Z_{t}, I_{t}, \vec{\tau}\right)^{\top} \mathbb{E}_{t}\left(\Delta \varepsilon_{t+\Delta t}^{\vec{\tau}}\right)-\left|W\left(Z_{t}, I_{t}, \vec{\tau}\right)^{\top}-\tilde{W}_{t-\Delta t}^{\top}\right| \vec{\delta}\right\}
$$

The second term in (11), $\left|W\left(Z_{t}, I_{t}, \vec{\tau}\right)^{\top}-\tilde{W}_{t-\Delta t}^{\top}\right| \vec{\delta}$, indicates the fact that we do not need to liquidate the whole portfolio from the previous period before trading on a new portfolio. To transit from the previous portfolio to a new one, we only need to trade on the difference $W\left(Z_{t}, I_{t}, \vec{\tau}\right)-\tilde{W}_{t-\Delta t}$, which is considerably more transaction-cost-efficient comparing to the trades with full liquidation at each period. So our trading strategy does not require a high turnover rate. However, holding a CDS contract for too long indeed increases the impact of the maturity effect and makes the approximation of $U\left(Z_{t}, \tau-\Delta t\right) \approx U\left(Z_{t}, \tau\right)$ less accurate. Therefore, we include some voluntary trading to ensure the holding period of any CDS contract is no longer than $2 \%$ of its initial maturity. The transaction cost induced by this voluntary trading has little effect on the optimal portfolio weight $\tilde{W}_{t}$ but will complicate the optimization. Therefore, we exclude the voluntary trading in the expected capital gain (11) and take it into consideration in the realized capital gain below to make the return calculation as realistic as possible.

\footnotetext{
${ }^{4}$ Notice that when $\tilde{W}_{t}=\mathbb{O}$ there is no trade at time $t$, which does occasionally happen from. Therefore even though the trading strategy monitors the dynamics of the CDS term structure weekly, it does not assume weekly trading in all CDS contracts. In other words, the inability to trade contracts weekly does not constrain the performance of our trading strategy in any way.
} 
The realized capital gain $C G_{t+\Delta t}^{a}$ is given by

$$
C G_{t+\Delta t}^{a}=s_{t}\left[\tilde{W}_{t}^{\top}\left(\text { Upfronts }_{t+\Delta t}^{\vec{\tau}-\Delta t}-\text { Upfronts }_{t}^{\vec{\tau}}\right)-\left(\left|\tilde{W}_{t}^{\top}-\tilde{W}_{t-\Delta t}^{\top}\right|+2\left|V T_{t}\right|^{\top}\right) \vec{\delta}\right]
$$

where $V T_{t}$ is the voluntary trading ensuring the holding period of any CDS contract is no longer than $2 \%$ of its initial maturity, and is determined by holding period distribution at $t-\Delta t$ and $\tilde{W}_{t}^{\top}$. Its detailed formula is given in Section 11.

As for the bid-ask spreads, we position ourselves as an end user and trade against dealers, therefore, the typical effective half-spreads that apply to five-year Upfronts are $14 \mathrm{bps}$, i.e., $\delta_{5 y r}=14 \mathrm{bps}$, as per Biswas et al. (2014). The sample period of Biswas et al. (2014) is from 2009 to 2014, which matches our out-of-sample period. Therefore, we believe by assuming $\delta_{5 y r}=14 \mathrm{bps}$ our results are based upon appropriate transaction costs and liquidity considerations in the CDS market during the period we focus on.

It is well known that the five-year CDS contracts are the most liquid among the contracts with different maturities. Therefore, the Upfronts with maturities other than five years are usually traded with higher bid-ask spreads. To take this into consideration, we assume

$$
\delta_{i}=\left\{\begin{array}{ll}
1.4 \delta_{5 y r} & \text { for } i=2,3,7, \text { and } 10 y r \\
1.5 \delta_{5 y r} & \text { for } i=1,15,20, \text { and } 30 y r
\end{array} .\right.
$$

This assumption is consistent with evidence of the CDS bid-ask spread term structure documented by Arakelyan and Serrano (2012). In the robustness tests, we vary the initial deposit and $\delta_{5 y r}$ using different values while keeping other $\delta_{i}$ according to (15), and see how they affect the profitability of our trading strategy.

\section{Voluntary Trading $V T$}

$V T_{t}$ is a $9 \times 1$ vector with each element being the amount of each CDS contract needed to trade at $t$ to ensure the holding periods shorter than $2 \%$ of the initial maturities. Based on the simulation 
results in Section 2, we find that when the holding period is shorter than $2 \% \tau,\left|U\left(Z_{t}, \tau-\Delta t\right)-U\left(Z_{t}, \tau\right)\right|$ $\left(\frac{\left|U\left(Z_{t}, \tau-\Delta t\right)-U\left(Z_{t}, \tau\right)\right|}{\left|U\left(Z_{t}, \tau\right)\right|}\right)$ is no more than two basis points $(0.17 \%)$ in average. $2 \%$ of the nine maturities are one, two, three, five, seven, 10, 16, 21, and 31 weeks, respectively.

Without loss of generality, we look at $i$ th element $V T_{t, i}$. To calculate $V T_{t, i}$, we first need to know the holding period distribution of $i$ th CDS contract at $t-\Delta t$ denoted by $H P_{t-\Delta t, i} . H P_{t-\Delta t, i}$ is characterized by $J_{t-\Delta t, i}$ pairs of holding position and period. Specifically,

$$
H P_{t-\Delta t, i} \triangleq\left\{\omega_{t-\Delta t, i}^{j}, P_{t-\Delta t, i}^{j}\right\}, j=1,2, \ldots, J_{t-\Delta t, i}
$$

where $\omega_{t-\Delta t, i}^{j}$ is the $j$ th holding position of $i$ th CDS that has been held for $P_{t-\Delta t, i}^{j}$ periods at $t-\Delta t$; $\sum_{j=1}^{J_{t-\Delta t, i}} \omega_{t-\Delta t, i}^{j}=\tilde{W}_{t-\Delta t, i} ; P_{t-\Delta t, i}^{n}>P_{t-\Delta t, i}^{m}$ for $m>n$. When $t=\Delta t$,

$$
H P_{\Delta t, i} \triangleq\left\{\tilde{W}_{\Delta t, i}, 0\right\}, J_{\Delta t, i}=1
$$

Given $H P_{t-\Delta t, i}$ and $\tilde{W}_{t, i}, H P_{t, i}$ can be determined in the following three steps. $V T_{t}$ will be given in the last step.

Step 1: Before trading $\tilde{W}_{t, i}$, each holding period in $H P_{t-\Delta t, i}$ increases by one period. That is $H P_{t-\Delta t, i}$ becomes

$$
\overline{H P}_{t, i} \triangleq\left\{\bar{\omega}_{t, i}^{j}, \bar{P}_{t, i}^{j}\right\}, j=1,2, \ldots, \bar{J}_{t, i}
$$

where $\bar{\omega}_{t, i}^{j}=\omega_{t-\Delta t, i}^{j}, \bar{P}_{t, i}^{j}=P_{t-\Delta t, i}^{j}+\Delta t$, and $\bar{J}_{t, i}=J_{t-\Delta t, i}$.

Step 2: After trading $\tilde{W}_{t, i}, \overline{H P}_{t, i}$ becomes

$$
\widehat{H P}_{t, i} \triangleq\left\{\widehat{\omega}_{t, i}^{j}, \widehat{P}_{t, i}^{j}\right\}, j=1,2, \ldots, \widehat{J}_{t, i}
$$

that is given for the following four mutually exclusive cases

Case 1, $\tilde{W}_{t, i} \tilde{W}_{t-\Delta t, i} \leq 0$ and $\tilde{W}_{t, i} \neq \tilde{W}_{t-\Delta t, i}, \widehat{\omega}_{t, i}^{1}=\tilde{W}_{t, i}, \widehat{P}_{t, i}^{1}=0, \widehat{J}_{t, i}=1$ 
Case $2, \tilde{W}_{t, i} \tilde{W}_{t-\Delta t, i}>0$ and $\left|\tilde{W}_{t, i}\right|>\left|\tilde{W}_{t-\Delta t, i}\right|, \widehat{J}_{t, i}=\bar{J}_{t, i}+1$,

$$
\left\{\begin{array}{l}
\widehat{\omega}_{t, i}^{j}=\bar{\omega}_{t, i}^{j}, \widehat{P}_{t, i}^{j}=\bar{P}_{t, i}^{j}, \text { for } j=1,2, \ldots, \bar{J}_{t, i} \\
\widehat{\omega}_{t, i}^{j}=\operatorname{sgn}\left(\tilde{W}_{t, i}\right)\left|\tilde{W}_{t, i}-\tilde{W}_{t-\Delta t, i}\right|, \widehat{P}_{t, i}^{j}=0, \text { for } j=\widehat{J}_{t, i}
\end{array}\right.
$$

where $\operatorname{sgn}(\bullet)$ is the sign function, it is +1 when $\bullet>0$ and -1 when $\bullet<0$.

Case 3, $\tilde{W}_{t, i} \tilde{W}_{t-\Delta t, i}>0$ and $\left|\tilde{W}_{t, i}\right|<\left|\tilde{W}_{t-\Delta t, i}\right|$, determine a cutoff point $k$ so that $k$ is the smallest integer that satisfies $\sum_{j=1}^{k}\left|\bar{\omega}_{t, i}^{j}\right| \geq\left|\tilde{W}_{t, i}-\tilde{W}_{t-\Delta t, i}\right|$. Mathematically,

$$
k=\arg \min _{m}\left\{\sum_{j=1}^{m}\left|\bar{\omega}_{t, i}^{j}\right|-\left|\tilde{W}_{t, i}-\tilde{W}_{t-\Delta t, i}\right|\right\}, \text { s.t., } \sum_{j=1}^{m}\left|\bar{\omega}_{t, i}^{j}\right| \geq\left|\tilde{W}_{t, i}-\tilde{W}_{t-\Delta t, i}\right|
$$

If $k \leq \bar{J}_{t, i}-1$, then $\widehat{J}_{t, i}=\bar{J}_{t, i}+1-k$,

$$
\begin{gathered}
\left\{\begin{array}{l}
\widehat{\omega}_{t, i}^{j}=\bar{\omega}_{t, i}^{j-1+k}, \text { for } j=2,3, \ldots, \widehat{J}_{t, i}, \\
\widehat{P}_{t, i}^{j}=\bar{P}_{t, i}^{j-1+k}, \text { for } j=1,2, \ldots, \widehat{J}_{t, i}, \\
\widehat{\omega}_{t, i}^{1}=\operatorname{sgn}\left(\tilde{W}_{t, i}\right)\left(\sum_{j=1}^{k}\left|\bar{\omega}_{t, i}^{j}\right|-\left|\tilde{W}_{t, i}-\tilde{W}_{t-\Delta t, i}\right|\right)
\end{array}\right. \\
\text { if } k=\bar{J}_{t, i} \text {, then } \widehat{J}_{t, i}=1, \widehat{\omega}_{t, i}^{1}=\operatorname{sgn}\left(\tilde{W}_{t, i}\right)\left(\sum_{j=1}^{k}\left|\bar{\omega}_{t, i}^{j}\right|-\left|\tilde{W}_{t, i}-\tilde{W}_{t-\Delta t, i}\right|\right), \text { and } \widehat{P}_{t, i}^{1}=\bar{P}_{t, i}^{k} .
\end{gathered}
$$

Case 4, $\tilde{W}_{t, i}=\tilde{W}_{t-\Delta t, i}, \widehat{H P}_{t, i}=\overline{H P}_{t, i}$.

Step 3: In this step $\widehat{H P}_{t, i}$ turns into $H P_{t, i} \triangleq\left\{\omega_{t, i}^{j}, P_{t, i}^{j}\right\}, j=1,2, \ldots, J_{t, i}$. The voluntary trading only happens when $\widehat{P}_{t, i}^{1}$ is longer than $2 \%$ of the $i$ th maturity. In this case,

$$
\left\{\begin{array}{l}
J_{t, i}=\widehat{J}_{t, i} \\
\omega_{t, i}^{j}=\widehat{\omega}_{t, i}^{j+1}, P_{t, i}^{j}=\widehat{P}_{t, i}^{j+1}, \text { for } j=1,2, \ldots, J_{t, i}-1, \quad \text { when } \widehat{P}_{t, i}^{\widehat{J}_{t, i}} \neq 0 \\
\omega_{t, i}^{J_{t, i}}=\widehat{\omega}_{t, i}^{1}, P_{t, i}^{J_{t, i}}=0
\end{array}\right.
$$


and

$$
\left\{\begin{array}{l}
J_{t, i}=\widehat{J}_{t, i}-1, \\
\omega_{t, i}^{j}=\widehat{\omega}_{t, i}^{j+1}, P_{t, i}^{j}=\widehat{P}_{t, i}^{j+1}, \text { for } j=1,2, \ldots, J_{t, i}-1, \quad \text { when } \widehat{P}_{t, i}^{\widehat{t}_{t, i}}=0 . \\
\omega_{t, i}^{J_{t, i}}=\widehat{\omega}_{t, i}^{1}+\widehat{\omega}_{t, i}^{\widehat{t}_{t, i}}, P_{t, i}^{J_{t, i}}=0,
\end{array}\right.
$$

Therefore $V T_{t, i}=\widehat{\omega}_{t, i}^{1}$ when $\widehat{P}_{t, i}^{1}$ is longer than $2 \%$ of the $i$ th maturity, and zero otherwise.

A graphical presentation of the above steps and voluntary trading using a numerical example is shown in Fig. 5.

\section{Holding period being $1 \%$ and $3 \%$ of initial maturity in VT}

The robustness of the results are tested with respect to the holding period in VT. We implement the strategy using two alternative cutoffs, $1 \%$ and $3 \%$, other than the $2 \%$ in the main text. $1 \%$ of the nine maturities are one, one, two, three, four, five, eight, 10, and 16 weeks, respectively. And 3\% of the nine maturities are two, three, five, eight, 11, 16, 23, 31, and 47 weeks. The results are shown in Table 6 and Table 7. From the tables, we can see that apparently the holding period has very limited impact on the results.

\section{More results on after fee performance}

\subsection{Case studies}

To further investigate how well the strategy applies to individual firms, we present results for 10 firms with different ratings and from different sectors. These 10 firms are Bell Canada Enterprises (BCE Inc, AA, Tel), TECO Energy (TECO Engy Inc, AA, Uti), Arrow Electric (Arrow Electrs Inc, BBB, Ind), Dell (Dell Inc, BBB, Tec), ConAgra Foods (ConAgra Foods Inc, BBB, CG), Prudential Financial (Prudential Finl Inc, BBB, Fin), Wendy's International (Wendys Intl Inc, BB, CS), Health Net (Health Net Inc, BB, HC), Huntsman International (Huntsman Intl llc, B, BM), and Dynegy Holdings (Dynegy Hldgs Inc, CCC, OG). 
The performance of the trading strategy on these 10 cases is summarized in Table 8 . We find that our strategy generates very large SR's for these 10 firms, either in-sample or out-of-sample. All of the 10 firms have out-of-sample SR's well above one. TECO Energy has the lowest out-of-sample SR of 1.25. In the three measures, the differences between the in-sample and out-of-sample performance are negligible in most cases, and we do not see a consistent pattern that the in-sample outperforms the outof-sample. This shows that the identified mispricings are robust and unlikely due to over-fitting of the data. Furthermore, the out-of-sample MDD's are all small (less than 22\%). Wendys Intl Inc has the lowest out-of-sample MDD of 5\%. BCE Inc has the largest out-of-sample MDD of 21\%. Therefore, our strategy not only has robust performance but also is associated with small downside risk.

Figures 6 and 7 present the in-sample and out-of-sample time series of the capital growth, which is

the cumulative capital gain, $\sum_{i=1}^{t / \Delta t} C G_{i \Delta t}^{a}$, over time for the 10 cases. We can see that for all cases the growth of the trading capital deployed is almost non-decreasing both in-sample and out-of-sample. The lines either rise or stay flat. The trading rules are dormant and wait for occasional opportunities. Figures 8 and 9 present the in-sample and out-of-sample time series of the capital gains, $C G_{t}^{a}$, over time for the 10 cases. Figures 10 and 11 show the histograms of the capital gain in Figures 8 and 9. From these figures, we see clearer evidence of the limited downside risk. Most of the time, the expected capital gain is smaller than the expected transaction cost induced by the bid-ask spreads. The trading rules in (12) thus recommend a zero position, leading to zero loss or gain. On infrequent occasions, the expected capital gain is much larger than the expected transaction cost, so the trading rule recommends large positions which work out profitably. Only on very rare occasions, the trading rule produces false signals that lead to losses. Overall, the results show that our trading strategy effectively sends useful trading signals that lead to significant profits with limited downside risk.

\subsection{Robustness with respect to transaction costs}

The analyses so far show that our trading strategy, after controlling for reasonable transaction costs, produces significant statistical arbitrage profits in out-of-sample tests. Nevertheless, for practical purposes, it is also helpful to see how the performance is affected by varying the transaction costs. 
We only consider the out-of-sample performance for the 10 cases in this section. The two sources of transaction costs are the effective bid-ask half-spreads, $\vec{\delta}$, and initial margin, IM. We let $\delta_{5 y r}$ range from four to 20 bps to vary $\vec{\delta}$ according to (15), and IM range from $\$ 0.25 \mathrm{M}$ to $\$ 3 \mathrm{M}$, then look at how the three performance measures change. The results of XR, SR, and MDD are plotted in Figures 12, 13, and 14, respectively. From Fig. 12, we see that the out-of-sample XR is very sensitive to both sources of transaction costs. Apparently, the larger is $\vec{\delta}$ the lower is XR. This is consistent with our intuition. We also find that in all cases the lower is IM the larger is XR. This is somewhat expected given the good performance of the strategy. So for the 10 cases the low IM do not induce any significant scale-down effects on the capital gain (see (14)), and instead they scale-up the returns.

As for the out-of-sample SR in Fig. 13, we find that the SR is highly sensitive to $\vec{\delta}$. This is also expected since a large $\vec{\delta}$ reduces the number of profitable trades and the magnitude of the profits, and increases the losses from false trading signals. For example, given $\mathrm{IM}=\$ 0.5 \mathrm{M}$ the $\mathrm{SR}$ of Arrow Electrs Inc decreases from 2.5 to 1.5 when $\delta_{5 y r}$ increases from four to $20 \mathrm{bps}$. However, the SR is relatively less sensitive to IM (except for the cases of BCE Inc and TECO Engy Inc). Unlike the XR and the MDD, the SR measures the risk adjusted excess returns, therefore it is somewhat invariant to the scaling effect of the initial cost.

We also observe a similar and intuitive relation between the out-of-sample MDD and IM in Fig. 14: the lower is IM the larger is MDD. Another interesting observation is about the relation between the MDD and $\delta_{5 y r}$. Except for two cases (Prudential Finl Inc and Huntsman Intl llc), we find that in general when IM is large the MDD increases as $\vec{\delta}$ decreases. This observation is less obvious but still understandable. A lower $\vec{\delta}$ increases the number of trading signals (both profitable and false). Since the MDD only measures the downside risk, when more false trading signals get executed larger draw-downs will of course be picked up in the MDD. This is probably why we see this inverse relation between the MDD and bid-ask spreads.

Overall we find that our trading strategy survives this stress test on transaction costs for the 10 cases. Even under the condition in which $\delta_{5 y r}$ is 20 bps (almost twice of the typical size reported in Biswas et al., 2014) our strategy still produces positive XR's and sizable SR's with very low MDD's. This exercise further verifies the robustness of our results. 


\section{Alternative random sample for case studies}

In this section, we present the case studies for 10 alternative firms that are randomly chosen. They are Bk of America, Fairfax Finl Hldgs Ltd, Radian Asset Assurn Inc, Sears Roebuck \& Co, Sempra Engy, Unvl Health Svcs Inc, JetBlue Awys Corp, Omnicare Inc, EOP Oper Ltd Pship, and Developers Diversified Rlty Corp. Their corresponding results are presented in Table 9 and Fig. 15 to Fig. 20. 
Table 1: Number of Firms in Different Rating and Industry Groups

This table presents a summary on the number of firms among different ratings and industries. The six ratings are $\mathrm{AA}, \mathrm{A}, \mathrm{BBB}, \mathrm{BB}, \mathrm{B}, \mathrm{CCC}$. The 10 sectors are basic materials(BM), consumer goods(CG), consumer services (CS), financials (Fin), health care (HC), industrials (Ind), oil \& gas (OG), technology (Tec), and telecommunication (Tel), utilities (Uti). Total number of firms across different ratings and industries are shown in the last column and the last row, respectively.

\begin{tabular}{cccccccc}
\hline & AA & A & BBB & BB & B & CCC & Total \\
\hline BM & 0 & 1 & 32 & 3 & 2 & 2 & 40 \\
CG & 2 & 0 & 38 & 12 & 4 & 10 & 66 \\
CS & 6 & 0 & 33 & 8 & 4 & 14 & 65 \\
Fin & 28 & 0 & 39 & 11 & 0 & 10 & 88 \\
HC & 0 & 0 & 30 & 3 & 2 & 1 & 36 \\
Ind & 19 & 0 & 54 & 0 & 0 & 3 & 76 \\
OG & 0 & 1 & 39 & 4 & 0 & 1 & 45 \\
Tec & 0 & 0 & 16 & 1 & 4 & 1 & 22 \\
Tel & 9 & 0 & 5 & 3 & 0 & 0 & 17 \\
Uti & 11 & 3 & 28 & 0 & 1 & 2 & 45 \\
Total & 75 & 5 & 314 & 45 & 17 & 44 & 500 \\
\hline
\end{tabular}


Table 2: Mean and Standard Deviation of CDS Spreads for Different Ratings

This table provides the mean and standard deviation of the CDS spreads in seven ratings (AAA, AA, A, BBB, BB, $\mathrm{B}$, and $\mathrm{CCC}$ ) for the nine maturities (1yr, 2yr, 3yr, 5yr, 7yr, 10yr, 15yr, 20yr, and 30yr). All numbers in the table are in percentages.

\begin{tabular}{|c|c|c|c|c|c|c|c|c|c|c|}
\hline & & $1 \mathrm{yr}$ & $2 \mathrm{yr}$ & $3 \mathrm{yr}$ & $5 \mathrm{yr}$ & $7 \mathrm{yr}$ & $10 \mathrm{yr}$ & $15 \mathrm{yr}$ & $20 \mathrm{yr}$ & $30 \mathrm{yr}$ \\
\hline \multirow{2}{*}{ AA } & Mean & 0.48 & 0.56 & 0.64 & 0.79 & 0.85 & 0.91 & 0.98 & 1.01 & 1.05 \\
\hline & Std. & 1.12 & 1.08 & 1.07 & 1.05 & 0.99 & 0.94 & 0.97 & 0.97 & 0.98 \\
\hline \multirow{2}{*}{ A } & Mean & 0.25 & 0.31 & 0.38 & 0.51 & 0.58 & 0.67 & 0.71 & 0.75 & 0.76 \\
\hline & Std. & 0.29 & 0.30 & 0.31 & 0.33 & 0.32 & 0.32 & 0.33 & 0.36 & 0.34 \\
\hline \multirow{2}{*}{ BBB } & Mean & 0.70 & 0.83 & 0.96 & 1.21 & 1.29 & 1.38 & 1.44 & 1.49 & 1.54 \\
\hline & Std. & 1.61 & 1.54 & 1.51 & 1.51 & 1.44 & 1.38 & 1.39 & 1.39 & 1.40 \\
\hline \multirow{2}{*}{ BB } & Mean & 2.13 & 2.39 & 2.62 & 3.02 & 3.08 & 3.12 & 3.17 & 3.20 & 3.24 \\
\hline & Std. & 5.56 & 5.01 & 4.65 & 4.24 & 3.92 & 3.62 & 3.44 & 3.36 & 3.29 \\
\hline \multirow{2}{*}{ B } & Mean & 4.13 & 4.58 & 5.04 & 5.74 & 5.74 & 5.70 & 5.70 & 5.69 & 5.65 \\
\hline & Std. & 10.60 & 8.77 & 7.86 & 6.92 & 6.17 & 5.54 & 5.42 & 5.05 & 4.76 \\
\hline \multirow{2}{*}{$\mathrm{CCC}$} & Mean & 6.90 & 7.22 & 7.43 & 7.68 & 7.37 & 7.06 & 6.82 & 6.69 & 6.63 \\
\hline & Std. & 14.84 & 12.88 & 11.77 & 10.51 & 9.43 & 8.45 & 7.73 & 7.42 & 7.02 \\
\hline
\end{tabular}


Table 3: Mean and Standard Deviation of CDS Spreads for Different Sectors

This table provides the mean and standard deviation of the CDS spreads in 10 sectors for the nine maturities (1yr, $2 \mathrm{yr}, 3 \mathrm{yr}, 5 \mathrm{yr}, 7 \mathrm{yr}, 10 \mathrm{yr}, 15 \mathrm{yr}, 20 \mathrm{yr}$, and 30yr). The 10 sectors are basic materials(BM), consumer goods(CG), consumer services (CS), financials (Fin), health care (HC), industrials (Ind), oil \& gas (OG), technology (Tec), and telecommunication (Tel), utilities (Uti). All numbers in the table are in percentages.

\begin{tabular}{|c|c|c|c|c|c|c|c|c|c|c|}
\hline & & $1 \mathrm{yr}$ & $2 \mathrm{yr}$ & $3 y r$ & $5 y r$ & $7 \mathrm{yr}$ & $10 \mathrm{yr}$ & $15 \mathrm{yr}$ & $20 \mathrm{yr}$ & $30 \mathrm{yr}$ \\
\hline \multirow{2}{*}{ BM } & Mean & 0.95 & 1.14 & 1.33 & 1.68 & 1.78 & 1.89 & 1.94 & 1.96 & 2.03 \\
\hline & Std. & 2.90 & 2.63 & 2.53 & 2.47 & 2.34 & 2.22 & 2.18 & 2.15 & 2.14 \\
\hline \multirow{2}{*}{ CG } & Mean & 2.00 & 2.16 & 2.31 & 2.57 & 2.60 & 2.62 & 2.64 & 2.67 & 2.72 \\
\hline & Std. & 7.77 & 6.66 & 6.09 & 5.54 & 5.07 & 4.66 & 4.50 & 4.33 & 4.20 \\
\hline \multirow{2}{*}{$\mathrm{CS}$} & Mean & 2.03 & 2.39 & 2.67 & 3.06 & 3.09 & 3.11 & 3.06 & 3.11 & 3.16 \\
\hline & Std. & 5.78 & 5.78 & 5.67 & 5.43 & 5.03 & 4.67 & 4.49 & 4.41 & 4.33 \\
\hline \multirow{2}{*}{ Fin } & Mean & 2.62 & 2.57 & 2.54 & 2.58 & 2.47 & 2.39 & 2.34 & 2.35 & 2.36 \\
\hline & Std. & 9.27 & 7.99 & 7.25 & 6.46 & 5.77 & 5.12 & 4.45 & 4.28 & 4.03 \\
\hline \multirow{2}{*}{$\mathrm{HC}$} & Mean & 0.59 & 0.75 & 0.90 & 1.17 & 1.26 & 1.34 & 1.39 & 1.43 & 1.48 \\
\hline & Std. & 1.02 & 1.21 & 1.35 & 1.58 & 1.57 & 1.55 & 1.55 & 1.58 & 1.59 \\
\hline \multirow{2}{*}{ Ind } & Mean & 0.84 & 1.02 & 1.18 & 1.48 & 1.57 & 1.65 & 1.68 & 1.73 & 1.78 \\
\hline & Std. & 2.02 & 1.96 & 1.94 & 2.00 & 1.92 & 1.84 & 1.83 & 1.82 & 1.83 \\
\hline \multirow{2}{*}{ OG } & Mean & 0.70 & 0.86 & 1.01 & 1.30 & 1.39 & 1.48 & 1.58 & 1.60 & 1.64 \\
\hline & Std. & 1.33 & 1.42 & 1.51 & 1.70 & 1.66 & 1.62 & 1.65 & 1.64 & 1.60 \\
\hline \multirow{2}{*}{ Tec } & Mean & 1.59 & 1.88 & 2.11 & 2.46 & 2.52 & 2.58 & 2.72 & 2.78 & 2.89 \\
\hline & Std. & 5.78 & 5.37 & 5.17 & 4.91 & 4.58 & 4.25 & 4.23 & 4.17 & 4.08 \\
\hline \multirow{2}{*}{ Tel } & Mean & 0.77 & 1.00 & 1.19 & 1.47 & 1.58 & 1.68 & 1.77 & 1.86 & 1.92 \\
\hline & Std. & 1.63 & 1.78 & 1.85 & 1.90 & 1.84 & 1.79 & 1.80 & 1.82 & 1.83 \\
\hline \multirow{2}{*}{ Uti } & Mean & 0.66 & 0.82 & 0.97 & 1.24 & 1.33 & 1.43 & 1.51 & 1.53 & 1.59 \\
\hline & Std. & 1.08 & 1.19 & 1.29 & 1.47 & 1.45 & 1.43 & 1.42 & 1.39 & 1.40 \\
\hline
\end{tabular}


Table 4: Summary of Simulation Results

This table summarizes the true and estimated model parameters. The mean, median, and standard deviation are based on 500 Monte Carlo simulations. In each simulation, four years of weekly Z's (matching the dates in our full sample period) are simulated. Given the true parameters, the simulated Z's, and the corresponding risk-free discount factors, the nine CDS upfronts with maturities of one, two, three, five, seven, 10, 15, 20, and 30 years are computed at every point in time.

\begin{tabular}{|c|c|c|c|c|c|c|c|c|}
\hline & & $\alpha$ & $\kappa$ & $\sigma$ & $c_{0}$ & $\alpha^{P} / \kappa^{P}$ & $\kappa^{P}$ & $y$ \\
\hline \multirow{4}{*}{ 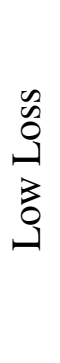 } & TRUE & 0.002 & -0.189 & 0.143 & 0.000 & 0.019 & 0.914 & 0.428 \\
\hline & Median & 0.003 & -0.180 & 0.120 & 0.001 & 0.020 & 3.392 & 0.305 \\
\hline & Mean & 0.003 & -0.173 & 0.118 & 0.001 & 0.019 & 3.657 & 0.305 \\
\hline & Std. & 0.001 & 0.025 & 0.011 & 0.001 & 0.009 & 2.149 & 0.039 \\
\hline \multirow{4}{*}{ 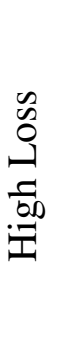 } & TRUE & 0.002 & -0.189 & 0.143 & 0.000 & 0.019 & 0.914 & 0.750 \\
\hline & Median & 0.002 & -0.187 & 0.138 & 0.001 & 0.013 & 5.018 & 0.684 \\
\hline & Mean & 0.002 & -0.186 & 0.137 & 0.000 & 0.014 & 5.999 & 0.682 \\
\hline & Std. & 0.000 & 0.010 & 0.004 & 0.004 & 0.008 & 3.791 & 0.032 \\
\hline
\end{tabular}


Table 5: Summary of the Trading Performance Using Alternative Bid-ask Term Structure

This table reports the first quarter $(\mathrm{Q} 1)$, median, third quarter $(\mathrm{Q} 3)$, and mean of three measures of the trading performance: annualized total excess return (XR), annualized Sharpe ratio (SR), and maximum drawdown (MDD). Panel (a) contains both the in-sample (upper subpanel) and out-of-sample (lower subpanel) results of the second half of the sample (Ju12008 - Oct2011), Panel (b) contains the full sample (Jan2005 - Oct2011) results. The benchmark risk-free return for XR is based on the U.S. treasury rate. On average it is $5 \%$ for the full sample, which is roughly the return of holding a 60 to 72 months zero coupon government bond issued at the beginning 2005 till its maturity, and $3 \%$ for the second half of the sample, which is roughly the return of holding a 40 months zero coupon government bond issued in Jul 2008 till its maturity.

(a) Second sub-sample (Jul2008 - Oct2011)

\begin{tabular}{|c|c|c|c|c|c|}
\hline & & Q1 & Median & Q3 & Mean \\
\hline \multirow{3}{*}{ 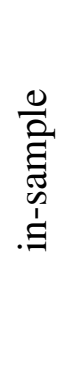 } & XR & 0.112 & 0.221 & 0.389 & 0.277 \\
\hline & SR & 1.118 & 1.469 & 1.785 & 1.398 \\
\hline & MDD & 0.038 & 0.065 & 0.112 & 0.094 \\
\hline \multirow{2}{*}{ 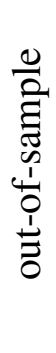 } & XR & 0.094 & 0.199 & 0.385 & 0.274 \\
\hline & MDD & 0.051 & 0.084 & 0.132 & 0.156 \\
\hline
\end{tabular}

(b) Full sample (Jan2005 - Oct2011)

\begin{tabular}{ccccc}
\hline & Q1 & Median & Q3 & Mean \\
\hline XR & 0.060 & 0.122 & 0.212 & 0.143 \\
& & & & \\
SR & 0.767 & 1.056 & 1.357 & 1.028 \\
& & & & \\
MDD & 0.039 & 0.064 & 0.110 & 0.101 \\
\hline
\end{tabular}


Table 6: Summary of the Trading Performance when holding period in VT is $1 \%$

This table reports the first quarter (Q1), median, third quarter (Q3), and mean of three measures of the trading performance: annualized total excess return (XR), annualized Sharpe ratio (SR), and maximum drawdown (MDD). Panel (a) contains both the in-sample (upper subpanel) and out-of-sample (lower subpanel) results of the second half of the sample (Ju12008 - Oct2011), Panel (b) contains the full sample (Jan2005 - Oct2011) results. The benchmark risk-free return for XR is based on the U.S. treasury rate. On average it is $5 \%$ for the full sample, which is roughly the return of holding a 60 to 72 months zero coupon government bond issued at the beginning 2005 till its maturity, and 3\% for the second half of the sample, which is roughly the return of holding a 40 months zero coupon government bond issued in Jul 2008 till its maturity.

(a) Second sub-sample (Jul2008 - Oct2011)

\begin{tabular}{|c|c|c|c|c|c|}
\hline & & Q1 & Median & Q3 & Mean \\
\hline \multirow{3}{*}{ 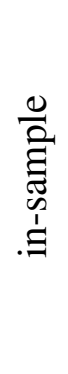 } & XR & 0.150 & 0.250 & 0.431 & 0.314 \\
\hline & SR & 1.220 & 1.586 & 1.962 & 1.549 \\
\hline & MDD & 0.049 & 0.080 & 0.137 & 0.108 \\
\hline \multirow{2}{*}{ 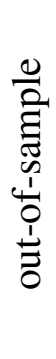 } & XR & 0.125 & 0.243 & 0.428 & 0.310 \\
\hline & MDD & 0.060 & 0.099 & 0.151 & 0.169 \\
\hline
\end{tabular}

(b) Full sample (Jan2005 - Oct2011)

\begin{tabular}{ccccc}
\hline & Q1 & Median & Q3 & Mean \\
\hline XR & 0.087 & 0.144 & 0.228 & 0.166 \\
& & & & \\
SR & 0.901 & 1.224 & 1.512 & 1.194 \\
& & & & \\
MDD & 0.050 & 0.076 & 0.127 & 0.119 \\
\hline
\end{tabular}


Table 7: Summary of the Trading Performance when holding period in VT is 3\%

This table reports the first quarter (Q1), median, third quarter (Q3), and mean of three measures of the trading performance: annualized total excess return (XR), annualized Sharpe ratio (SR), and maximum drawdown (MDD). Panel (a) contains both the in-sample (upper subpanel) and out-of-sample (lower subpanel) results of the second half of the sample (Ju12008 - Oct2011), Panel (b) contains the full sample (Jan2005 - Oct2011) results. The benchmark risk-free return for XR is based on the U.S. treasury rate. On average it is $5 \%$ for the full sample, which is roughly the return of holding a 60 to 72 months zero coupon government bond issued at the beginning 2005 till its maturity, and 3\% for the second half of the sample, which is roughly the return of holding a 40 months zero coupon government bond issued in Jul 2008 till its maturity.

(a) Second sub-sample (Jul2008 - Oct2011)

\begin{tabular}{|c|c|c|c|c|c|}
\hline & & Q1 & Median & Q3 & Mean \\
\hline \multirow{3}{*}{ 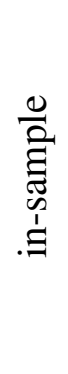 } & XR & 0.169 & 0.279 & 0.460 & 0.342 \\
\hline & SR & 1.399 & 1.702 & 2.081 & 1.702 \\
\hline & MDD & 0.041 & 0.066 & 0.110 & 0.091 \\
\hline \multirow{2}{*}{ 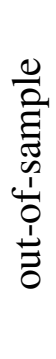 } & XR & 0.156 & 0.270 & 0.459 & 0.345 \\
\hline & MDD & 0.047 & 0.073 & 0.116 & 0.143 \\
\hline
\end{tabular}

(b) Full sample (Jan2005 - Oct2011)

\begin{tabular}{ccccc}
\hline & Q1 & Median & Q3 & Mean \\
\hline XR & 0.097 & 0.159 & 0.243 & 0.179 \\
& & & & \\
SR & 1.016 & 1.334 & 1.622 & 1.311 \\
& & & & \\
MDD & 0.042 & 0.065 & 0.103 & 0.102 \\
\hline
\end{tabular}


Table 8: After Fee Performance Summary for 10 Cases

This table summarizes the performance of the strategy for the 10 firms in the second half of the sample (Jul2008 - Oct2011). Columns four to seven report the minimal (Min), median (Mdn), maximal (Max), and mean (Mn) of the weekly raw returns. Columns eight to 10 report the annualized total excess return (XR), annualized Sharpe ratio (SR), and maximal draw-down (MDD). Panel (a) contains in-sample results, panel (b) contains out-of-sample results.

(a) In-sample

\begin{tabular}{|c|c|c|c|c|c|c|c|c|c|}
\hline & Rating & Sector & Min & Mdn & $\operatorname{Max}$ & $\mathrm{Mn}$ & XR & SR & MDD \\
\hline BCE Inc & $\mathrm{AA}$ & Tel & -0.23 & 0.00 & 0.35 & 0.01 & 0.50 & 1.45 & 0.23 \\
\hline TECO Engy Inc & $\mathrm{AA}$ & Uti & -0.08 & 0.00 & 0.18 & 0.01 & 0.44 & 2.14 & 0.08 \\
\hline Arrow Electrs Inc & BBB & Ind & -0.09 & 0.00 & 0.15 & 0.01 & 0.70 & 2.73 & 0.10 \\
\hline Dell Inc & BBB & Tec & -0.08 & 0.00 & 0.14 & 0.01 & 0.32 & 2.24 & 0.08 \\
\hline ConAgra Foods Inc & $\mathrm{BBB}$ & CG & -0.08 & 0.00 & 0.10 & 0.01 & 0.23 & 1.87 & 0.08 \\
\hline Prudential Finl Inc & $\mathrm{BBB}$ & Fin & -0.09 & 0.00 & 0.23 & 0.01 & 0.39 & 2.02 & 0.09 \\
\hline Wendys Intl Inc & $\mathrm{BB}$ & $\mathrm{CS}$ & -0.06 & 0.00 & 0.10 & 0.01 & 0.42 & 2.82 & 0.06 \\
\hline Health Net Inc & $\mathrm{BB}$ & $\mathrm{HC}$ & -0.03 & 0.00 & 0.21 & 0.01 & 0.39 & 2.34 & 0.05 \\
\hline Huntsman Intl llc & $\mathrm{B}$ & $\mathrm{BM}$ & -0.12 & 0.00 & 0.30 & 0.01 & 0.63 & 1.99 & 0.12 \\
\hline Dynegy Hldgs Inc & $\mathrm{CCC}$ & OG & -0.03 & 0.00 & 0.14 & 0.01 & 0.52 & 2.66 & 0.03 \\
\hline \multicolumn{10}{|c|}{ (b) Out-of-sample } \\
\hline & Rating & Sector & Min & Mdn & Max & $\mathrm{Mn}$ & $\mathrm{XR}$ & SR & MDD \\
\hline BCE Inc & AA & Tel & -0.21 & 0.00 & 0.34 & 0.01 & 0.47 & 1.57 & 0.21 \\
\hline TECO Engy Inc & AA & Uti & -0.07 & 0.00 & 0.94 & 0.01 & 0.66 & 1.25 & 0.07 \\
\hline Arrow Electrs Inc & $\mathrm{BBB}$ & Ind & -0.13 & 0.00 & 0.31 & 0.01 & 0.68 & 2.23 & 0.16 \\
\hline Dell Inc & $\mathrm{BBB}$ & $\mathrm{Tec}$ & -0.09 & 0.00 & 0.12 & 0.01 & 0.34 & 2.37 & 0.10 \\
\hline ConAgra Foods Inc & $\mathrm{BBB}$ & $\mathrm{CG}$ & -0.18 & 0.00 & 0.20 & 0.01 & 0.29 & 1.33 & 0.18 \\
\hline Prudential Finl Inc & $\mathrm{BBB}$ & Fin & -0.07 & 0.00 & 0.18 & 0.01 & 0.35 & 1.91 & 0.07 \\
\hline Wendys Intl Inc & $\mathrm{BB}$ & $\mathrm{CS}$ & -0.05 & 0.00 & 0.09 & 0.01 & 0.44 & 2.96 & 0.05 \\
\hline Health Net Inc & $\mathrm{BB}$ & $\mathrm{HC}$ & -0.10 & 0.00 & 0.61 & 0.02 & 0.95 & 1.53 & 0.13 \\
\hline Huntsman Intl llc & $\mathrm{B}$ & $\mathrm{BM}$ & -0.07 & 0.00 & 0.55 & 0.01 & 0.70 & 1.52 & 0.07 \\
\hline Dynegy Hldgs Inc & $\mathrm{CCC}$ & $\mathrm{OG}$ & -0.11 & 0.00 & 0.22 & 0.01 & 0.47 & 2.03 & 0.12 \\
\hline
\end{tabular}


Table 9: Performance Summary for 10 Alternative Cases

This table summarizes the performance of the strategy for the 10 firms in the second half of the sample (Jul2008 - Oct2011). Columns four to seven report the minimal (Min), median (Mdn), maximal (Max), and mean (Mn) of the weekly raw returns. Columns eight to 10 report the annualized total excess return (XR), annualized Sharpe ratio (SR), and maximal draw-down (MDD). Panel (a) contains in-sample results, panel (b) contains out-of-sample results.

(a) In-sample

\begin{tabular}{|c|c|c|c|c|c|c|c|c|c|}
\hline & Rating & Sector & Min & Mdn & Max & $\mathrm{Mn}$ & XR & SR & MDD \\
\hline Bk of America NA & AA & Fin & -0.05 & 0.00 & 0.10 & 0.01 & 0.24 & 2.12 & 0.07 \\
\hline Fairfax Finl Hldgs Ltd & BBB & Fin & -0.13 & 0.00 & 0.81 & 0.02 & 1.10 & 1.71 & 0.20 \\
\hline Radian Asset Assurn Inc & $\mathrm{CCC}$ & Fin & -0.13 & 0.00 & 0.30 & 0.01 & 0.70 & 2.17 & 0.13 \\
\hline Sears Roebuck \& Co & $\mathrm{CCC}$ & CG & -0.02 & 0.00 & 0.19 & 0.01 & 0.39 & 2.20 & 0.08 \\
\hline Sempra Engy & A & Uti & -0.04 & 0.00 & 0.08 & 0.01 & 0.34 & 2.47 & 0.04 \\
\hline Unvl Health Svcs Inc & BBB & $\mathrm{HC}$ & -0.04 & 0.00 & 0.19 & 0.01 & 0.61 & 2.28 & 0.05 \\
\hline JetBlue Awys Corp & $\mathrm{CCC}$ & CS & -0.09 & 0.00 & 0.27 & 0.01 & 0.72 & 2.43 & 0.09 \\
\hline Omnicare Inc & BB & CS & -0.04 & 0.00 & 0.32 & 0.02 & 0.91 & 2.45 & 0.05 \\
\hline Huntsman Intl llc & BBB & Fin & -0.07 & 0.00 & 0.18 & 0.01 & 0.36 & 1.90 & 0.08 \\
\hline Developers Diversified Rlty Corp & BB & Fin & -0.21 & 0.00 & 0.52 & 0.02 & 0.85 & 1.73 & 0.21 \\
\hline \multicolumn{10}{|c|}{ (b) Out-of-sample } \\
\hline & Rating & Sector & Min & Mdn & Max & $\mathrm{Mn}$ & XR & SR & MDD \\
\hline Bk of America NA & AA & Fin & -0.05 & 0.00 & 0.13 & 0.01 & 0.21 & 1.56 & 0.08 \\
\hline Fairfax Finl Hldgs Ltd & BBB & Fin & -0.10 & 0.00 & 0.64 & 0.02 & 1.12 & 1.76 & 0.10 \\
\hline Radian Asset Assurn Inc & $\mathrm{CCC}$ & Fin & -0.12 & 0.00 & 0.25 & 0.01 & 0.52 & 1.81 & 0.12 \\
\hline Sears Roebuck \& Co & $\mathrm{CCC}$ & CG & -0.06 & 0.00 & 0.19 & 0.01 & 0.29 & 1.59 & 0.07 \\
\hline Sempra Engy & A & Uti & -0.03 & 0.00 & 0.11 & 0.01 & 0.29 & 2.46 & 0.04 \\
\hline Unvl Health Svcs Inc & BBB & $\mathrm{HC}$ & -0.03 & 0.00 & 0.20 & 0.01 & 0.64 & 2.29 & 0.04 \\
\hline JetBlue Awys Corp & $\mathrm{CCC}$ & CS & -0.11 & 0.00 & 0.33 & 0.02 & 0.84 & 2.32 & 0.11 \\
\hline Omnicare Inc & BB & $\mathrm{CS}$ & -0.04 & 0.00 & 0.22 & 0.01 & 0.65 & 2.39 & 0.05 \\
\hline Huntsman Intl llc & BBB & Fin & -0.07 & 0.00 & 0.18 & 0.01 & 0.34 & 1.96 & 0.07 \\
\hline Developers Diversified Rlty Corp & $\mathrm{BB}$ & Fin & -0.04 & 0.00 & 0.26 & 0.01 & 0.75 & 2.31 & 0.04 \\
\hline
\end{tabular}


Figure 1: Sensitivity and Partial Derivative of CDS Upfronts to the Loss Rate $y$

This figure shows the sensitivity (left and upper panel) and partial derivative (other panels) of CDS upfronts to the loss rate $y$. The sensitivity is calculated by varying $y$ from $10 \%$ to $100 \%$ while the value of $y Z$ is fixed at 52 bps for CDS upfronts. The partial derivative is calculated for the CDS upfront that is a function of $y$ and $y Z$ instead of $y$ and $Z$ with respect to $y$ by varying $\alpha$ (right and upper panel), $\kappa$ (middle and left panel), $\sigma$ (middle and right panel), $y Z$ (lower and left panel), and $y$ (lower and right panel). The maturities of the CDS upfronts are two, four, 10, and 20 years. The fixed parameters are displayed in the title of each panel.
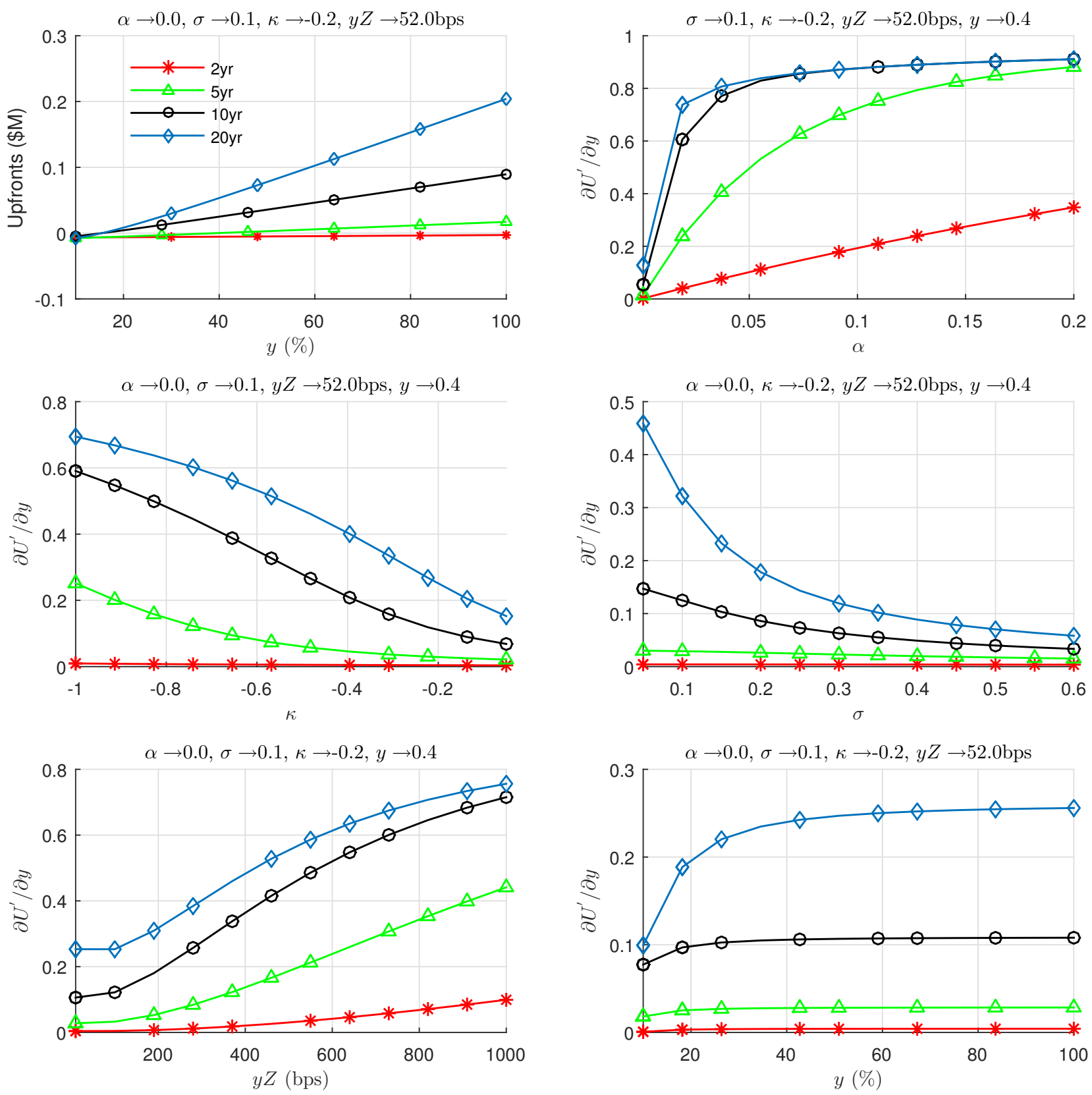
Figure 2: Aggregate returns when $\Delta t=$ one month: histograms and cumulative path

The first (second) panel shows the histogram of the aggregate monthly returns from July 2008 to October 2011 (July 2009 to October 2011). The third panel shows the cumulative returns over time. All returns are monthly and the unit of x-axes in the first and second panel, and y-axis in the third panel is percent.
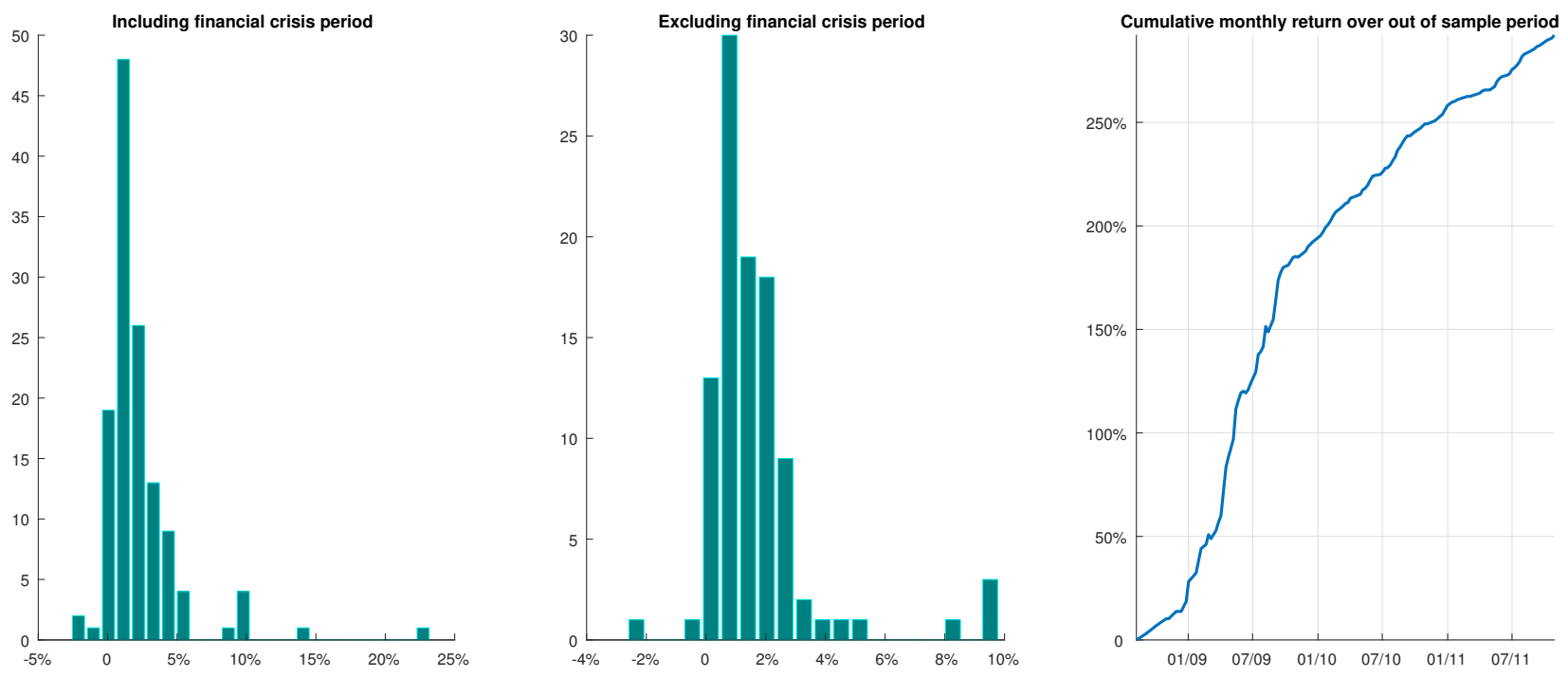

Figure 3: Aggregate returns when alternative interest rates are used (OIS and IRS)

The first (second) panel shows the histogram of the aggregate monthly returns from July 2008 to October 2011 (July 2009 to October 2011). The third panel shows the cumulative returns over time. All returns are monthly and the unit of x-axes in the first and second panel, and y-axis in the third panel is percent.
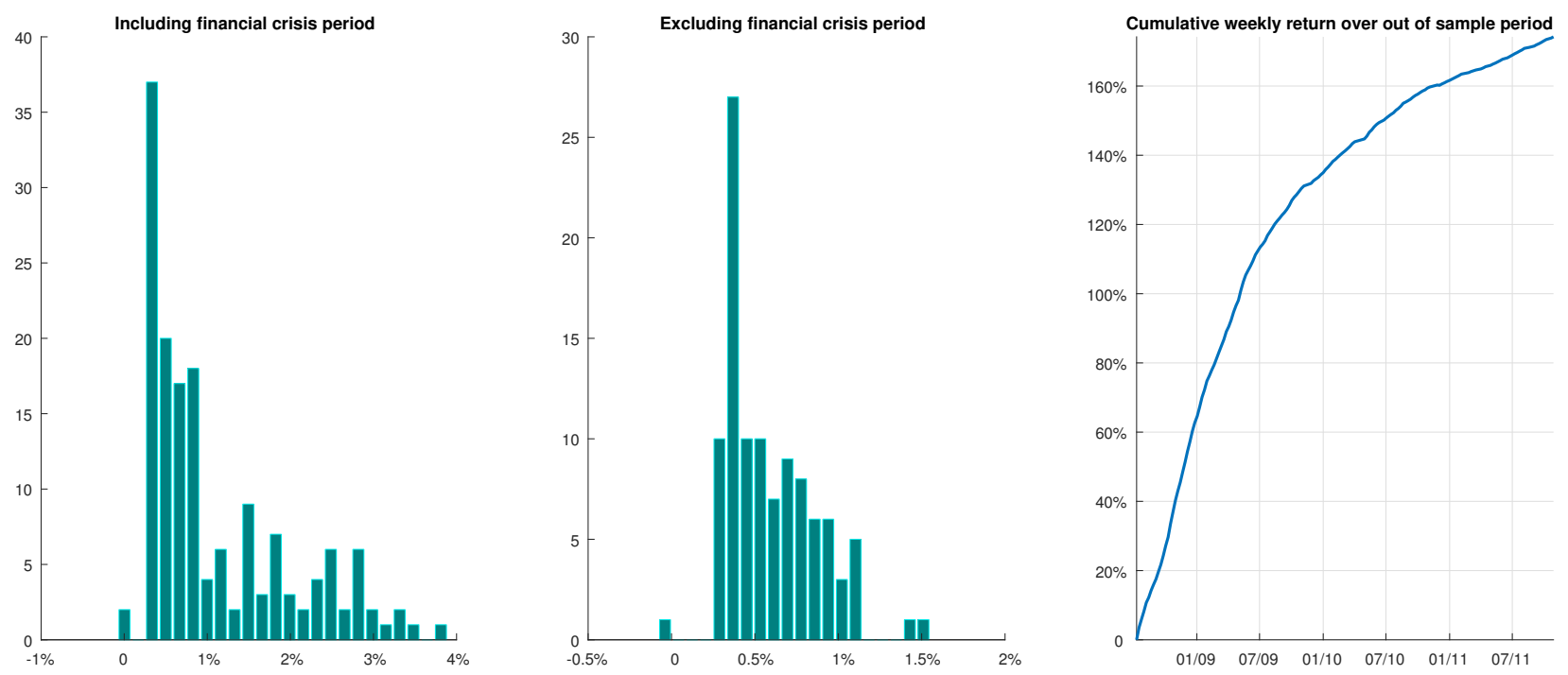
Figure 4: Aggregate returns when financial firms are excluded

The first (second) panel shows the histogram of the aggregate monthly returns from July 2008 to October 2011 (July 2009 to October 2011). The third panel shows the cumulative returns over time. All returns are monthly and the unit of x-axes in the first and second panel, and y-axis in the third panel is percent.
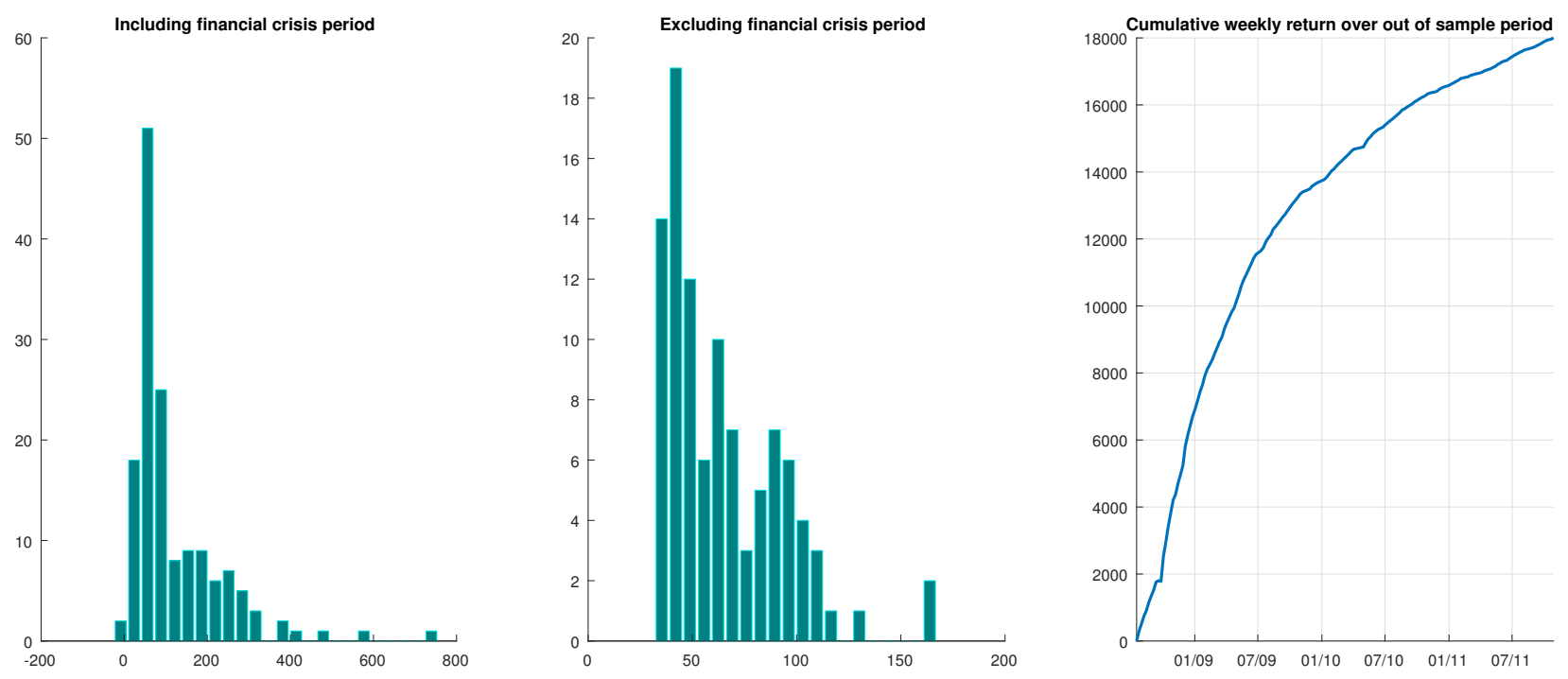


\section{Figure 5: Graphical example of voluntary trading}

This figure graphically shows the three steps and voluntary trading detailed in Section 11 using a numerical example. In the example, $\bar{W}_{t-\Delta t, i}=10,2 \% \tau_{i}=2$ weeks, $\tilde{W}_{t, i}$ is $-5,10.5,5$, and 10 for cases 1 to 4 , respectively. $V T_{t, i}$ is calculated in Step 3 .

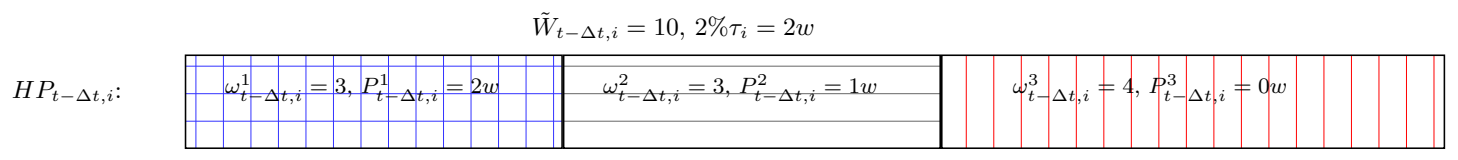

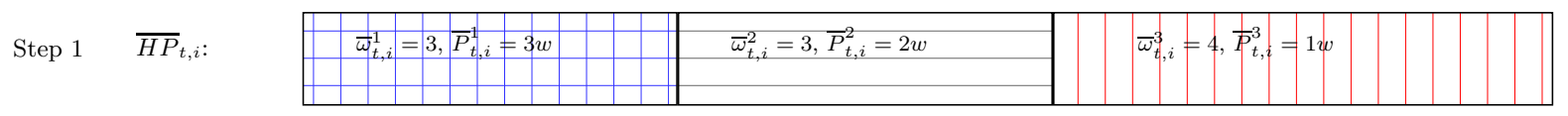
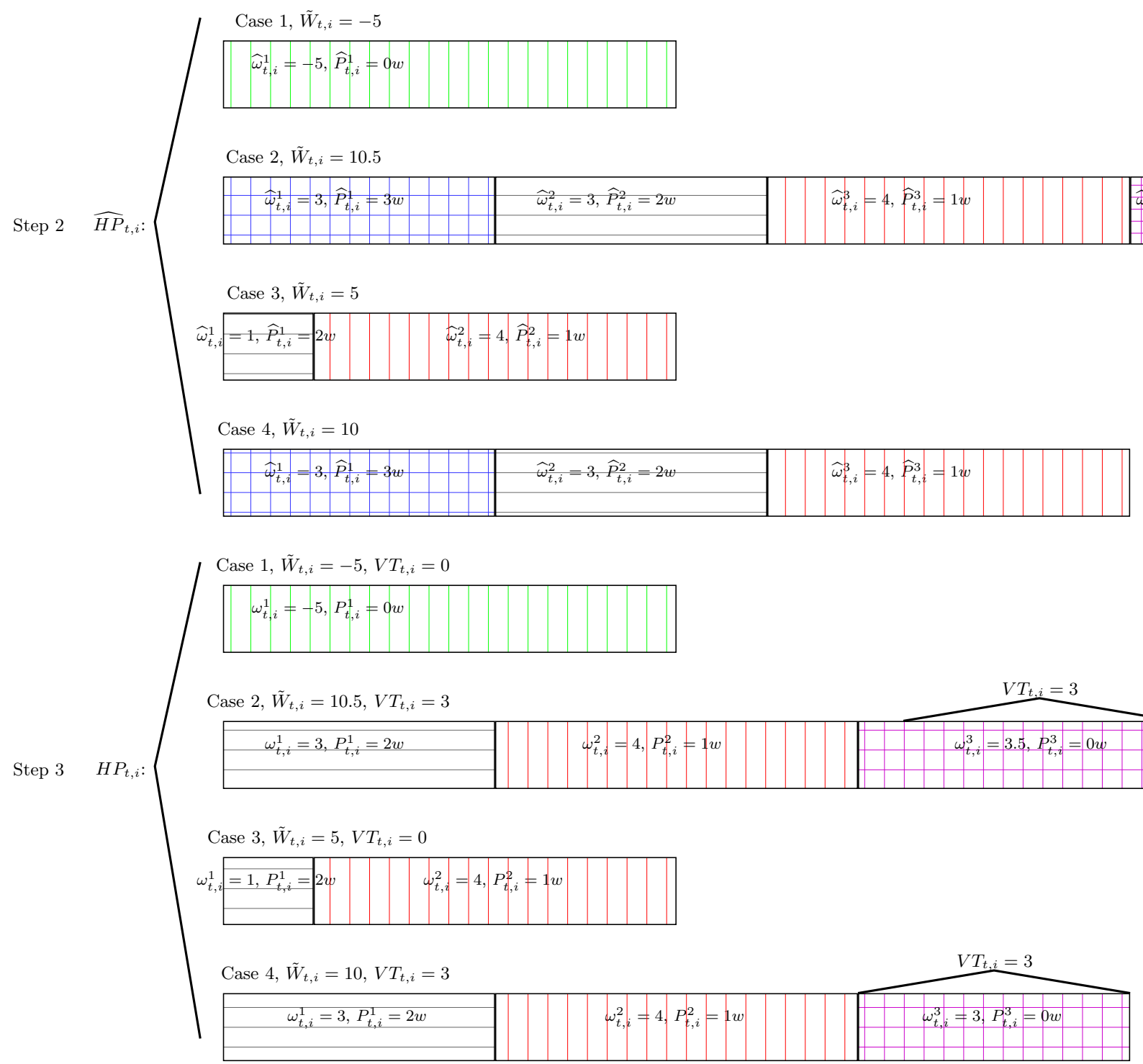
Figure 6: Time Series of In-sample Capital Growth in 10 Cases

This figure shows the in-sample capital growth, which is the cumulative capital gain over time, of the 10 firms in the second half of the sample (Jul2008 - Oct2011). Their names, ratings, and sectors are shown in the subtitles. The initial capital deposit is $\$ 1 \mathrm{M}$.
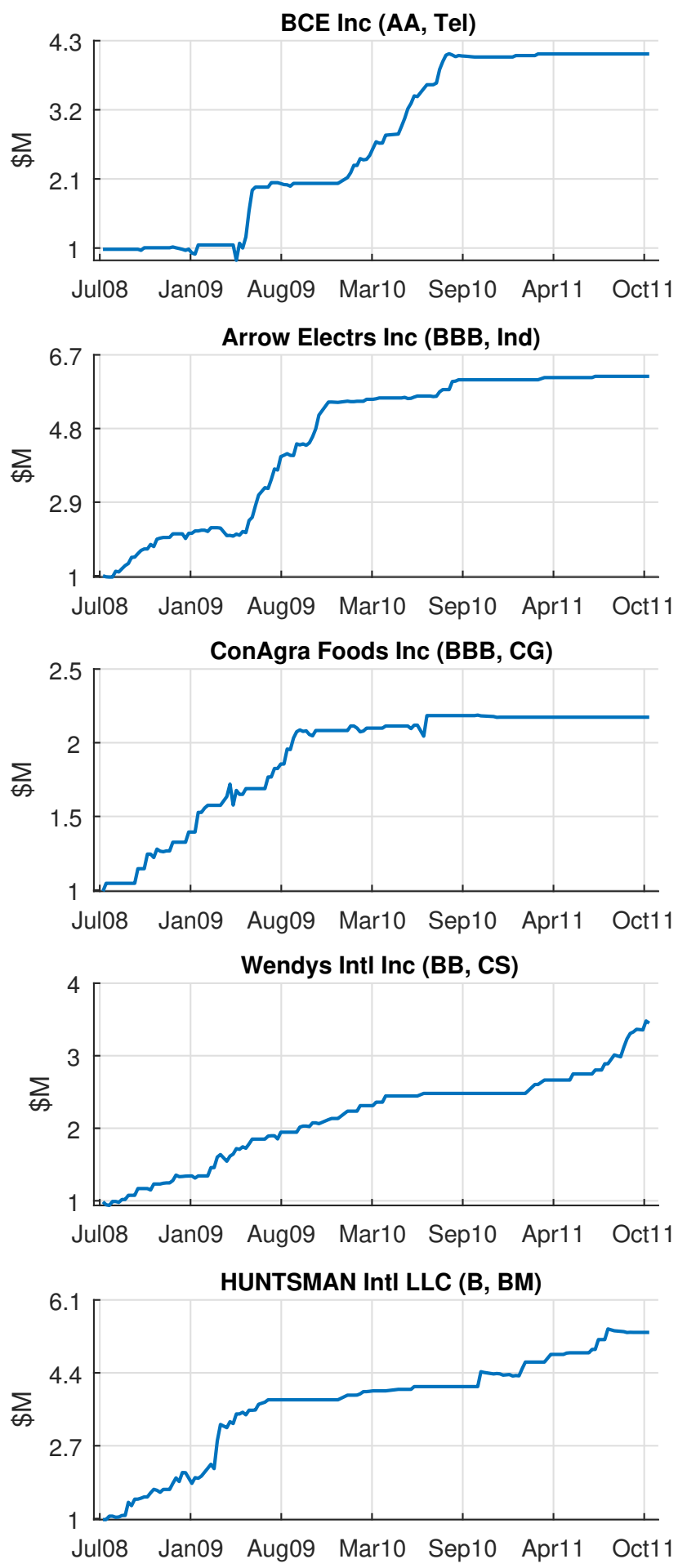
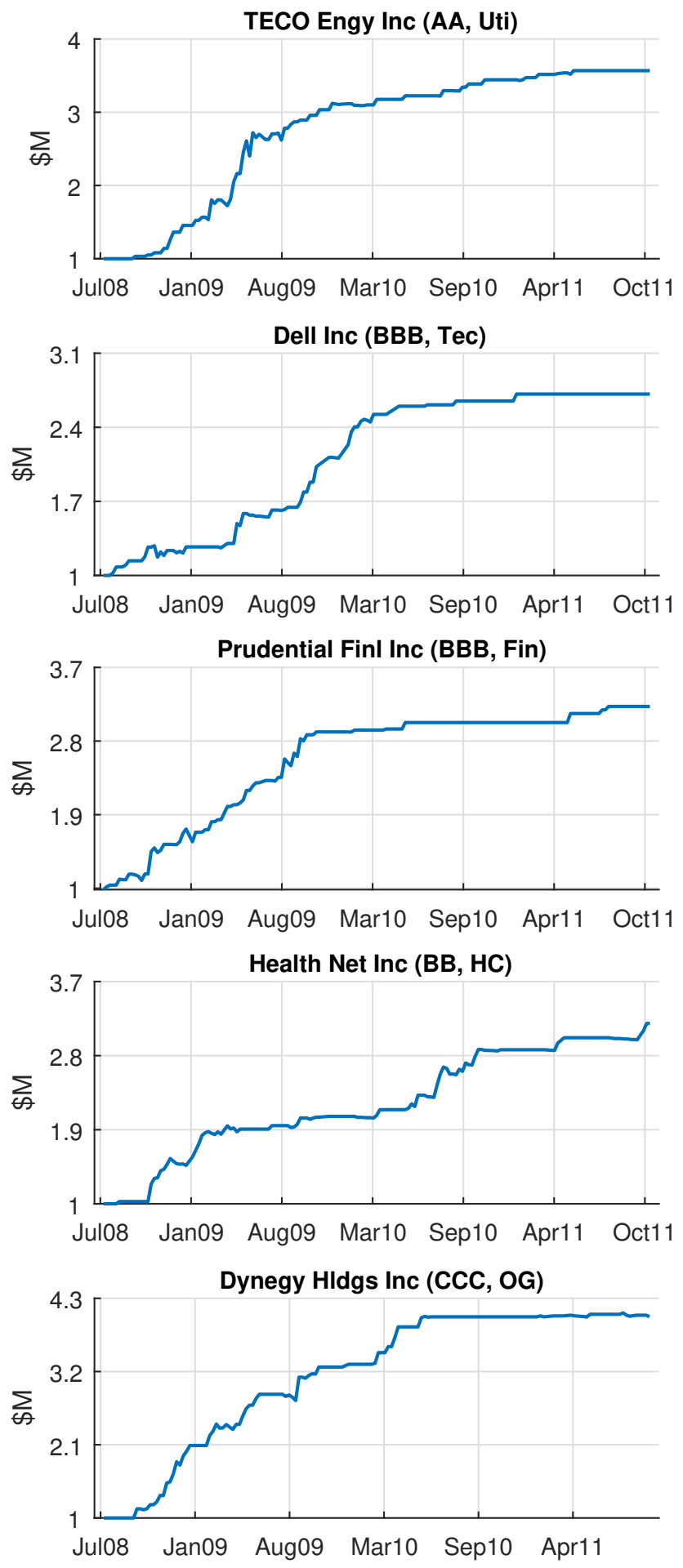
Figure 7: Time Series of Out-of-sample Capital Growth in 10 Cases

This figure shows the out-of-sample capital growth, which is the cumulative capital gain over time, of the 10 firms in the second half of the sample (Jul2008 - Oct2011). Their names, ratings, and sectors are shown in the subtitles. The initial capital deposit is $\$ 1 \mathrm{M}$.
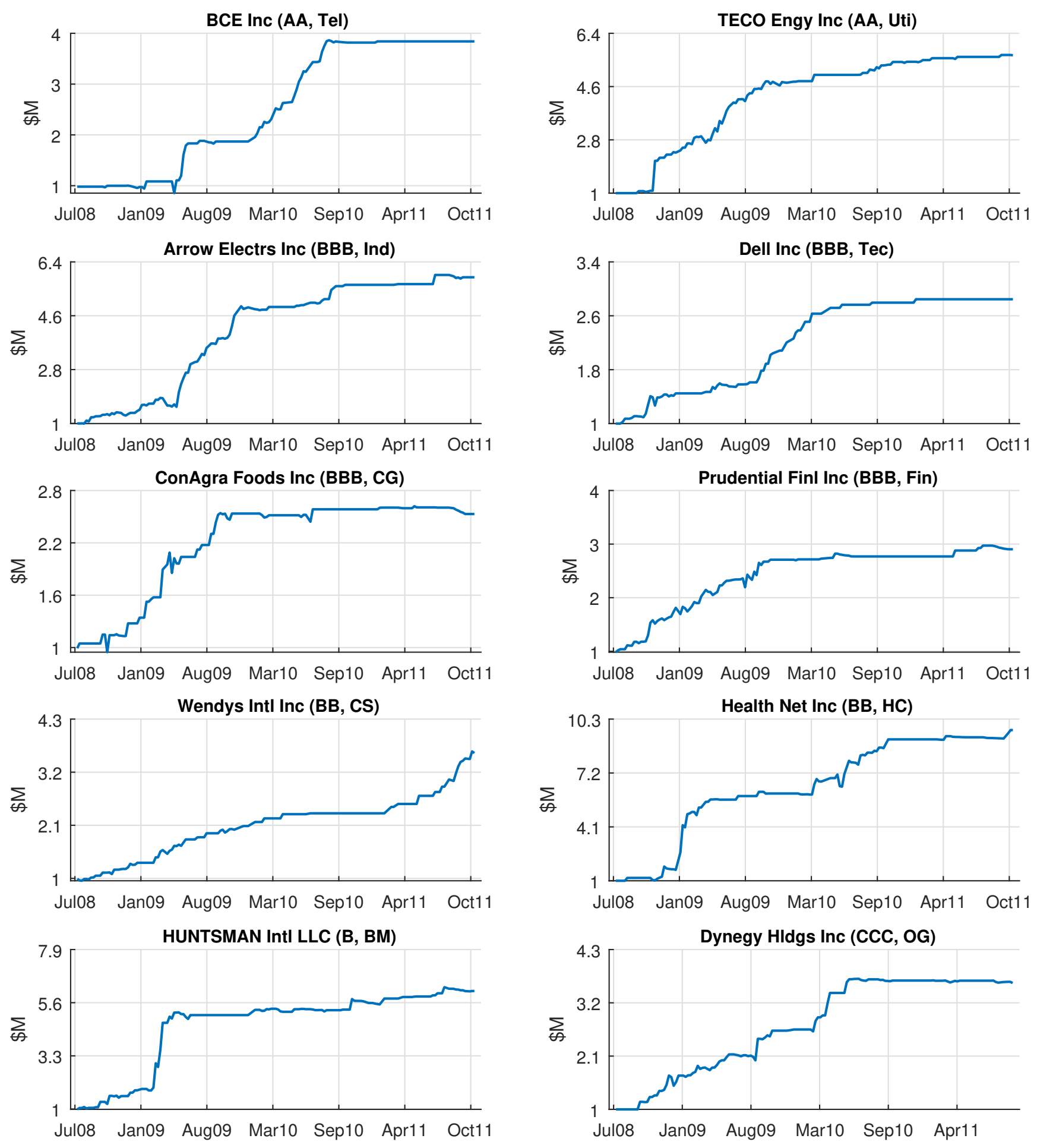
Figure 8: Time Series of In-sample Capital Gain and Loss in 10 Cases

This figure shows the in-sample capital gain and loss of the 10 firms in the second half of the sample (Jul2008 - Oct2011). Their names, ratings, and sectors are shown in the subtitles.
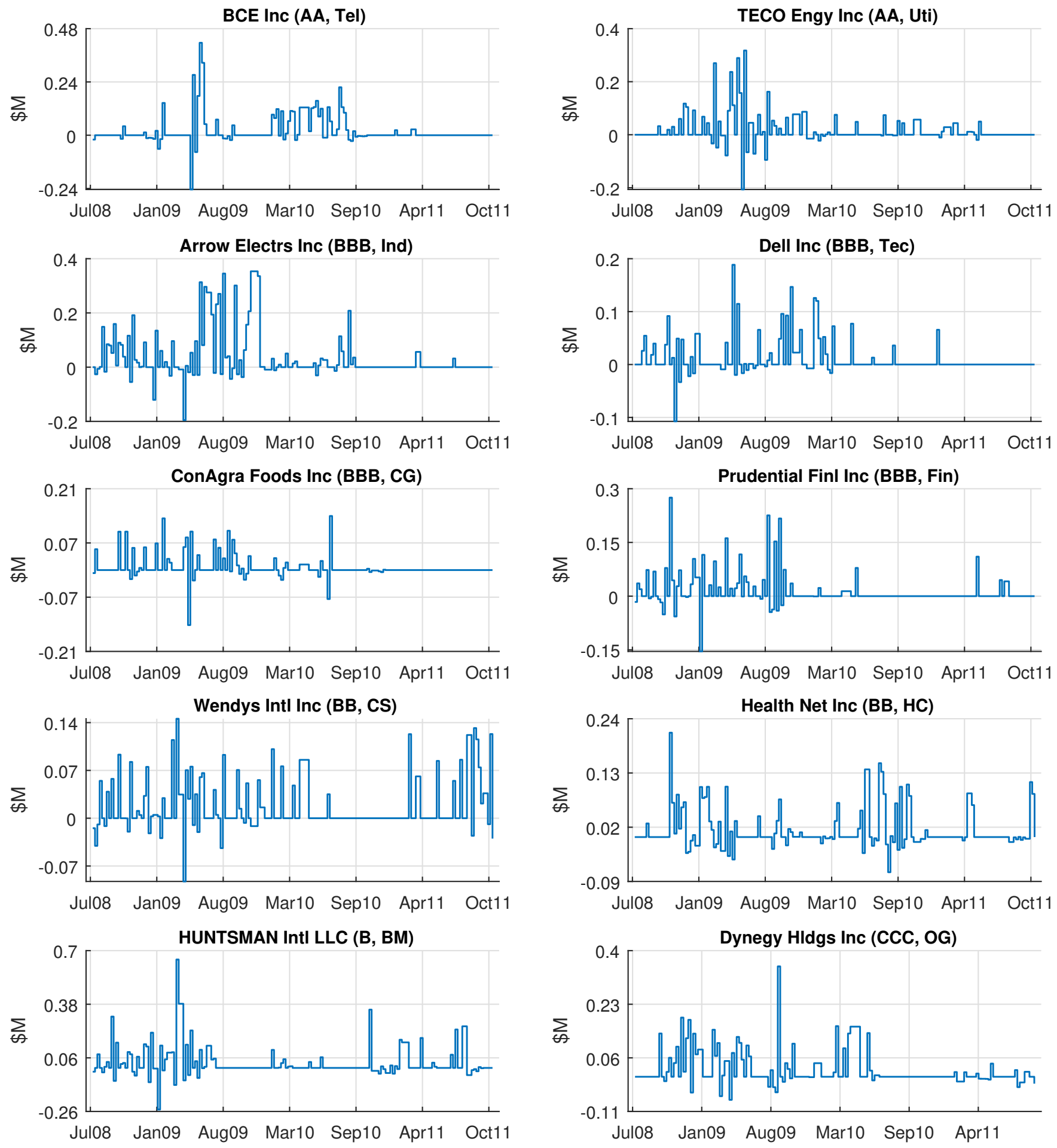
Figure 9: Time Series of Out-of-sample Capital Gain and Loss in 10 Cases

This figure shows the out-of-sample capital gain and loss of the 10 firms in the second half of the sample (Jul2008 - Oct2011). Their names, ratings, and sectors are shown in the subtitles.
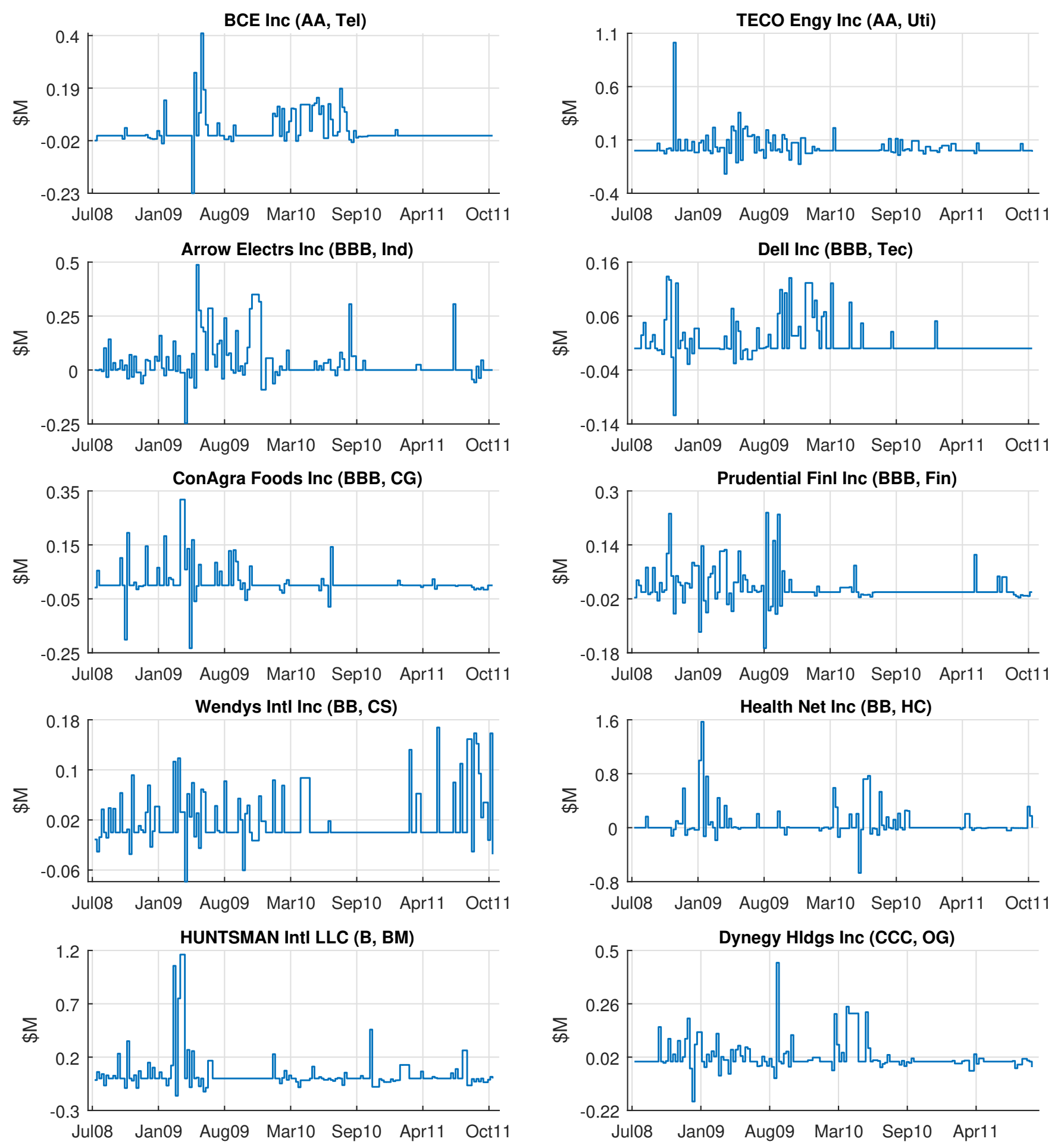
Figure 10: Histograms of In-sample Weekly Returns in 10 Cases

This figure shows the histograms of the in-sample weekly raw returns of the 10 firms in the second half of the sample (Jul2008 - Oct2011). Their names, ratings, and sectors are shown in the subtitles.

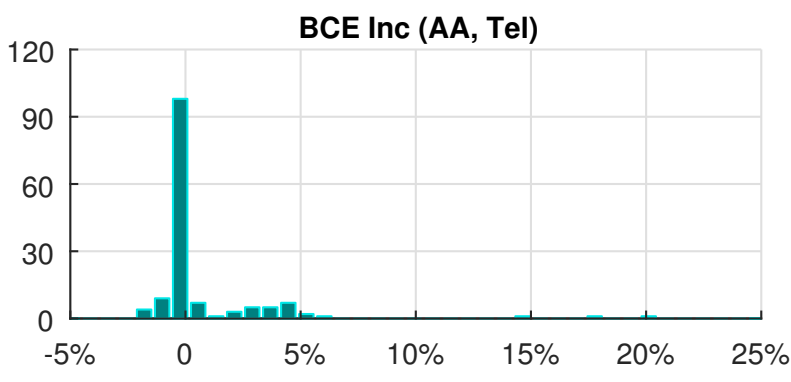

Arrow Electrs Inc (BBB, Ind)

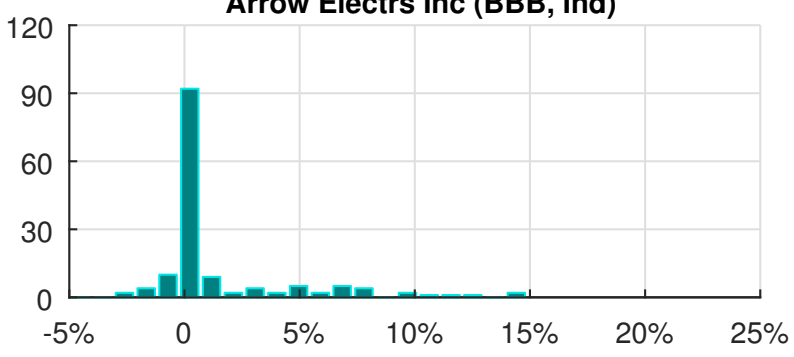

ConAgra Foods Inc (BBB, CG)
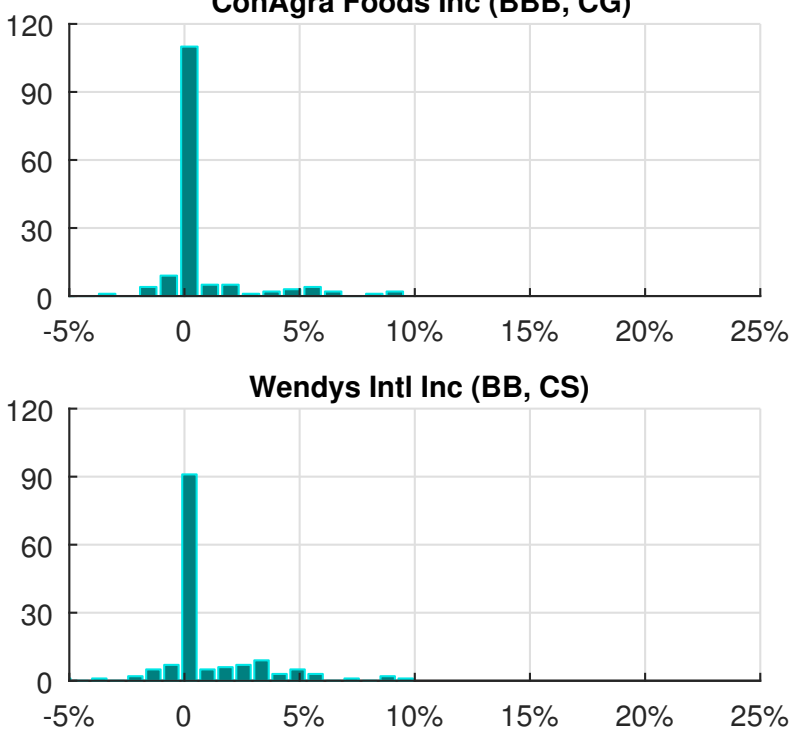

HUNTSMAN IntI LLC (B, BM)

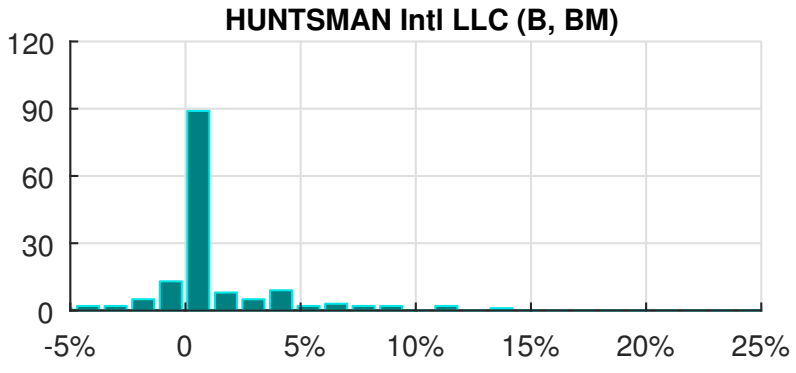

TECO Engy Inc (AA, Uti)

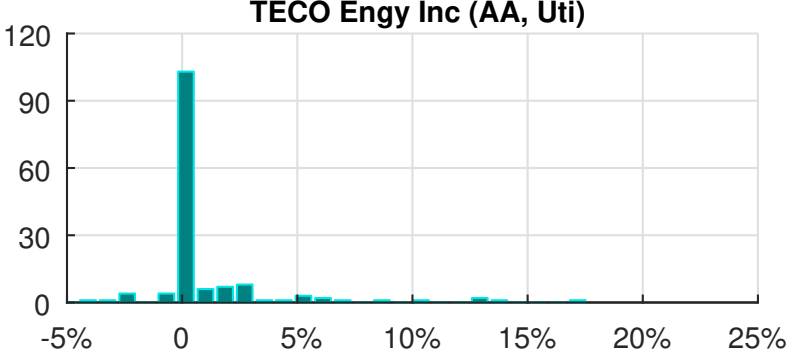

Dell Inc (BBB, Tec)
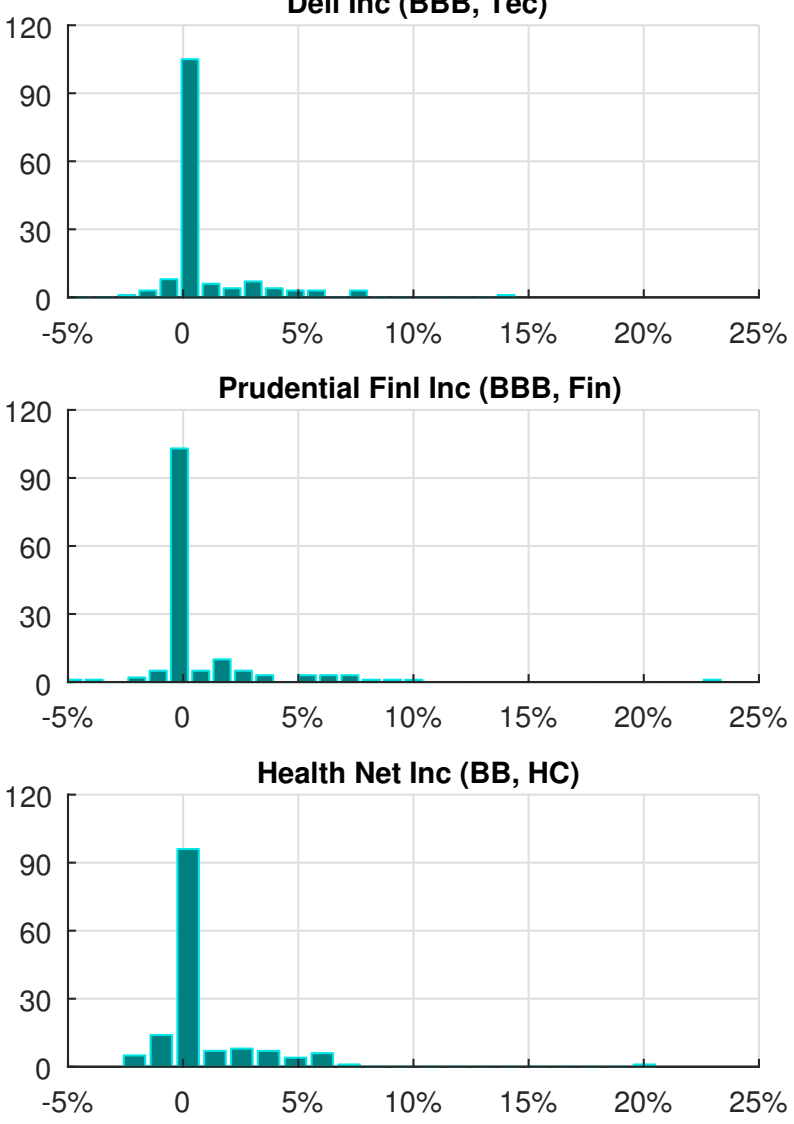

Dynegy HIdgs Inc (CCC, OG)

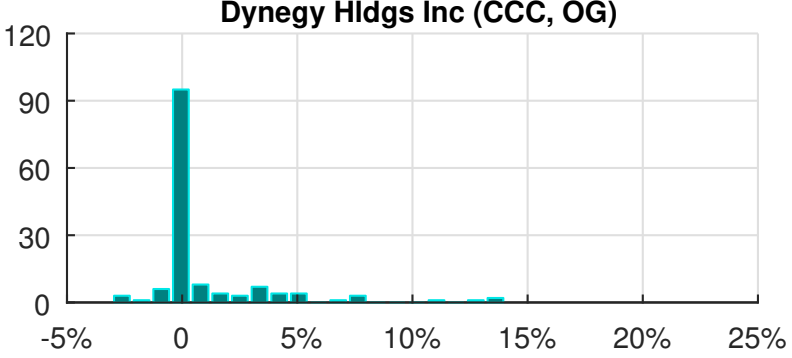


Figure 11: Histograms of Out-of-sample Weekly Returns in 10 Cases

This figure shows the histograms of the out-of-sample weekly raw returns of the 10 firms in the second half of the sample (Jul2008 - Oct2011). Their names, ratings, and sectors are shown in the subtitles.

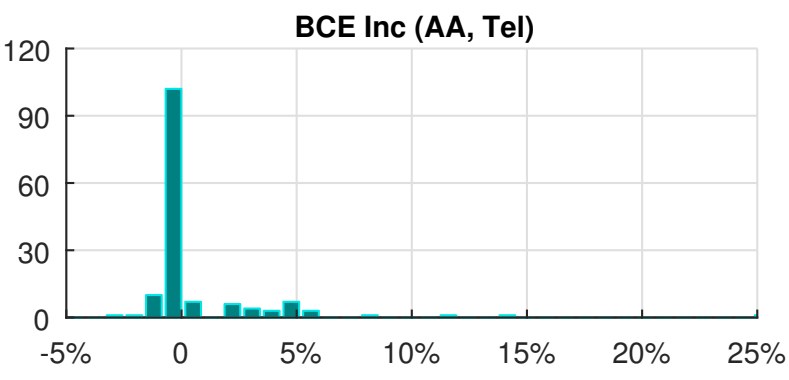

Arrow Electrs Inc (BBB, Ind)

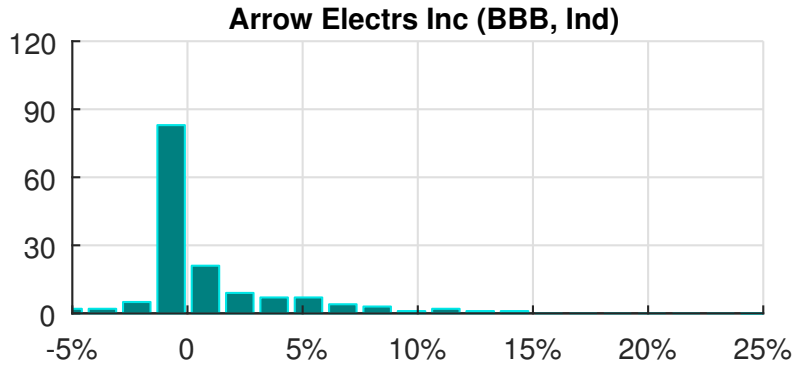

ConAgra Foods Inc (BBB, CG)
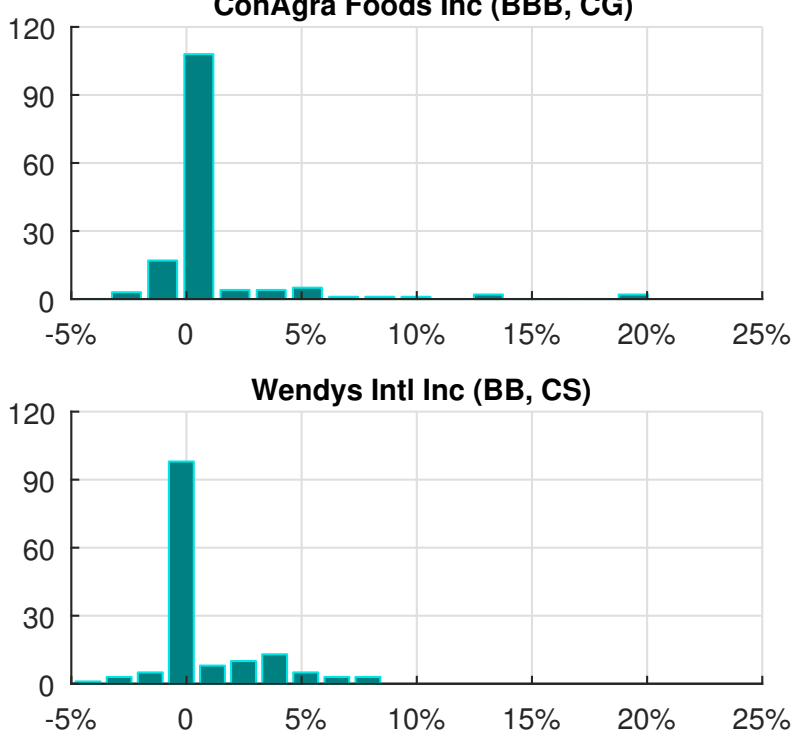

HUNTSMAN IntI LLC (B, BM)

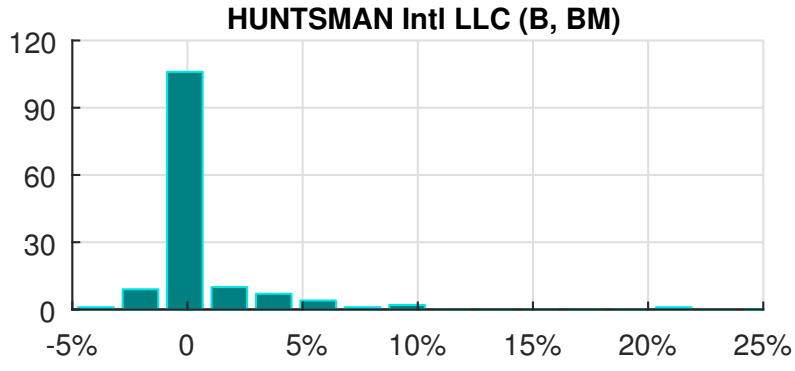

TECO Engy Inc (AA, Uti)

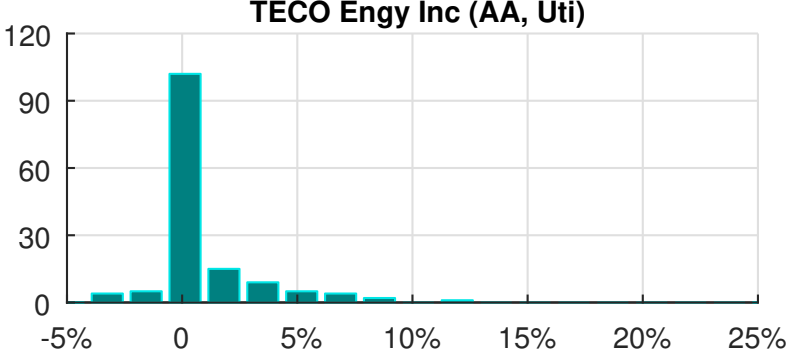

Dell Inc (BBB, Tec)

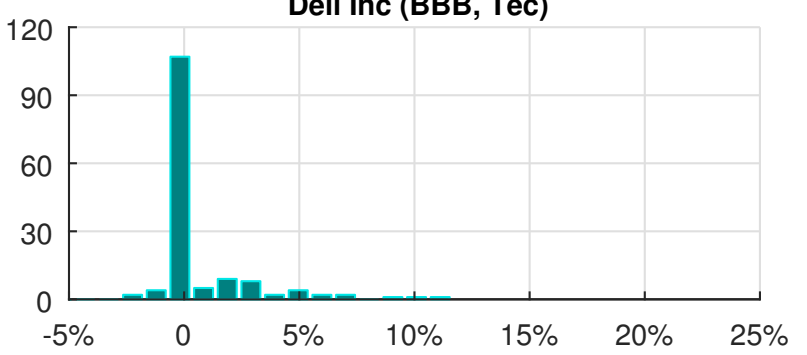

Prudential Finl Inc (BBB, Fin)
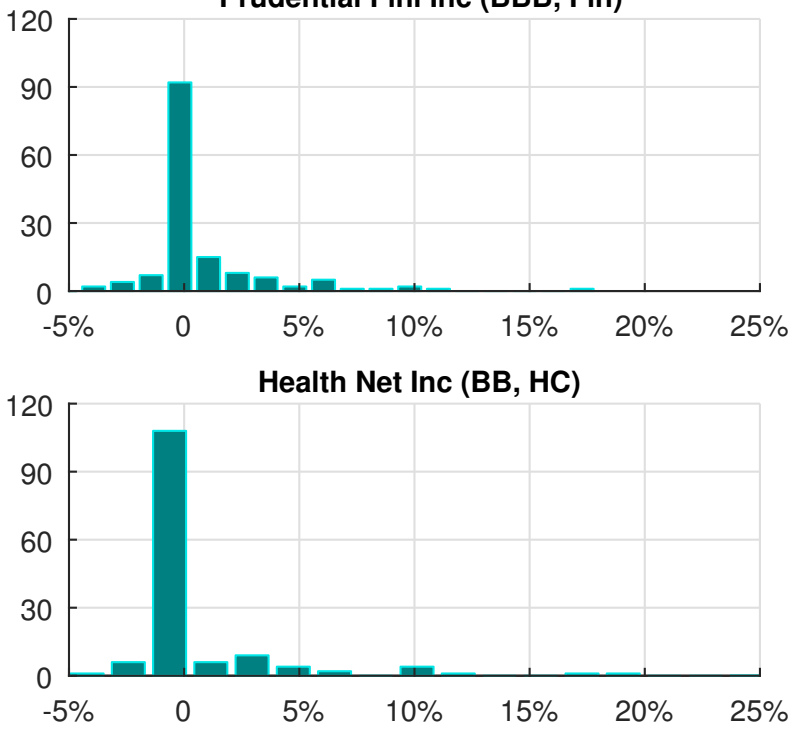

Dynegy HIdgs Inc (CCC, OG)

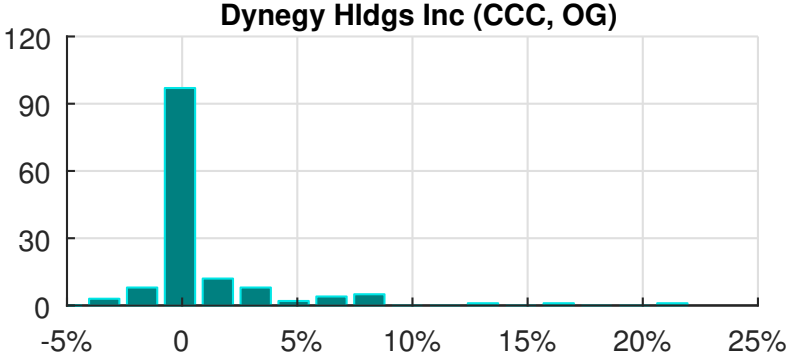


Figure 12: Out-of-sample Annualized Excess Return (XR) given Varying Transaction Costs in 10 Cases

This figure shows the out-of-sample annualized excess return (XR) of the 10 firms in the second half of the sample (Jul2008 - Oct2011) under different transaction costs. Their names, ratings, and sectors are shown in the subtitles. The bid-ask spreads vary from four to 24 basis points. The initial costs vary from $\$ 1 \mathrm{M}$ to $\$ 4.5 \mathrm{M}$.
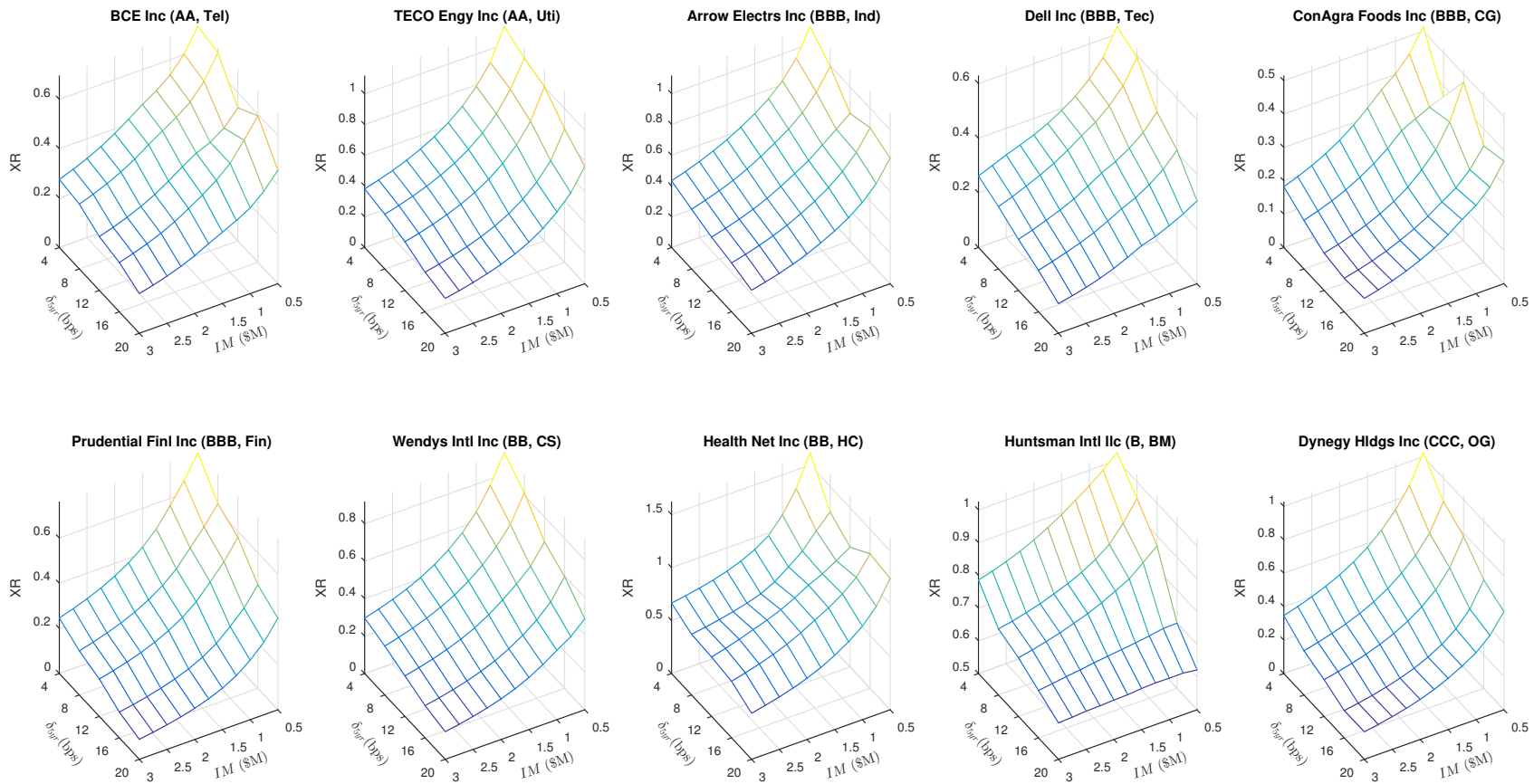
Figure 13: Out-of-sample Annualized Sharpe Ratio (SR) given Varying Transaction Costs in 10 Cases

This figure shows the out-of-sample annualized Sharpe ratio (SR) of the 10 firms in the second half of the sample (Jul2008 - Oct2011) under different transaction costs. Their names, ratings, and sectors are shown in the subtitles. The bid-ask spreads vary from four to 24 basis points. The initial costs vary from $\$ 1 \mathrm{M}$ to $\$ 4.5 \mathrm{M}$.
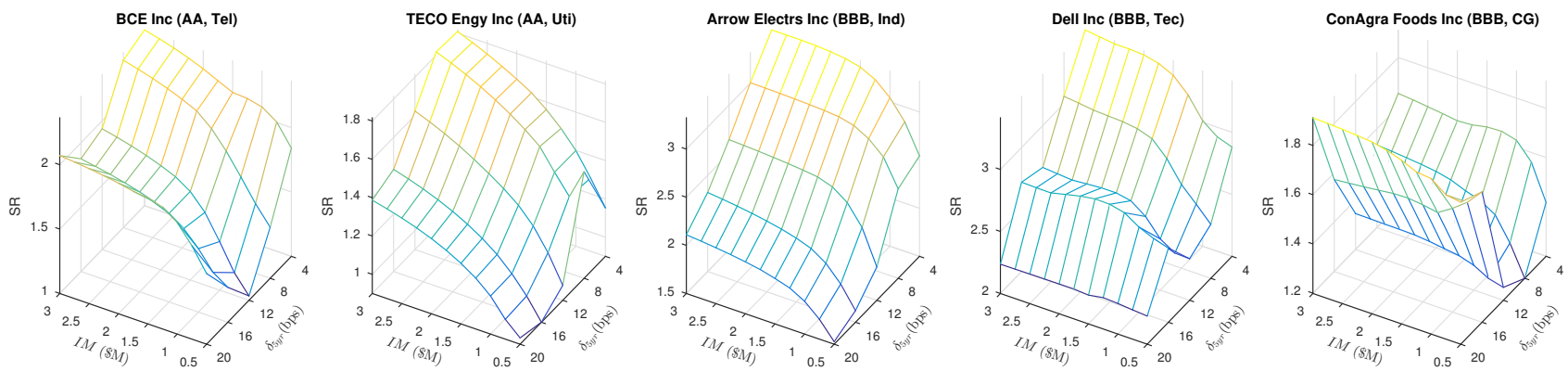

Prudential Finl Inc (BBB, Fin)

Wendys Intl Inc (BB, CS)

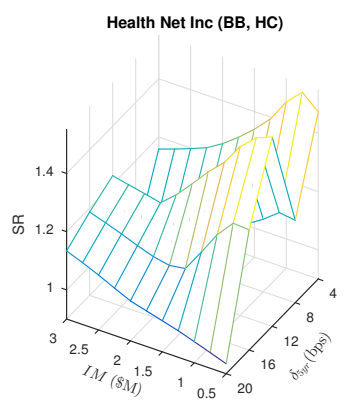

Huntsman IntI IIc (B, BM)

Dynegy HIdgs Inc (CCC, OG)
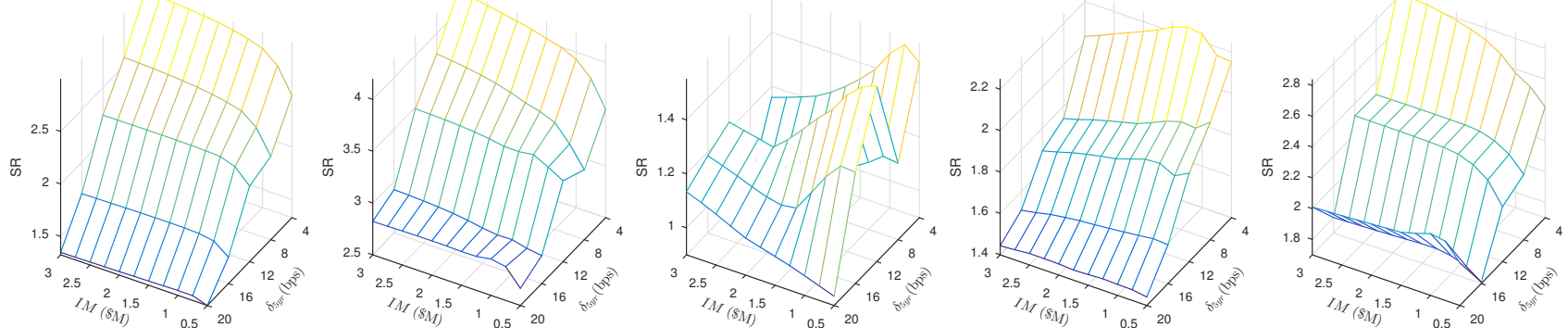
Figure 14: Out-of-sample Maximum Draw-down (MDD) given Varying Transaction Costs in 10 Cases

This figure shows the out-of-sample maximum draw-down (MDD) of the 10 firms in the second half of the sample (Jul2008 - Oct2011) under different transaction costs. Their names, ratings, and sectors are shown in the subtitles. The bid-ask spreads vary from four to 24 basis points. The initial costs vary from $\$ 1 \mathrm{M}$ to $\$ 4.5 \mathrm{M}$.
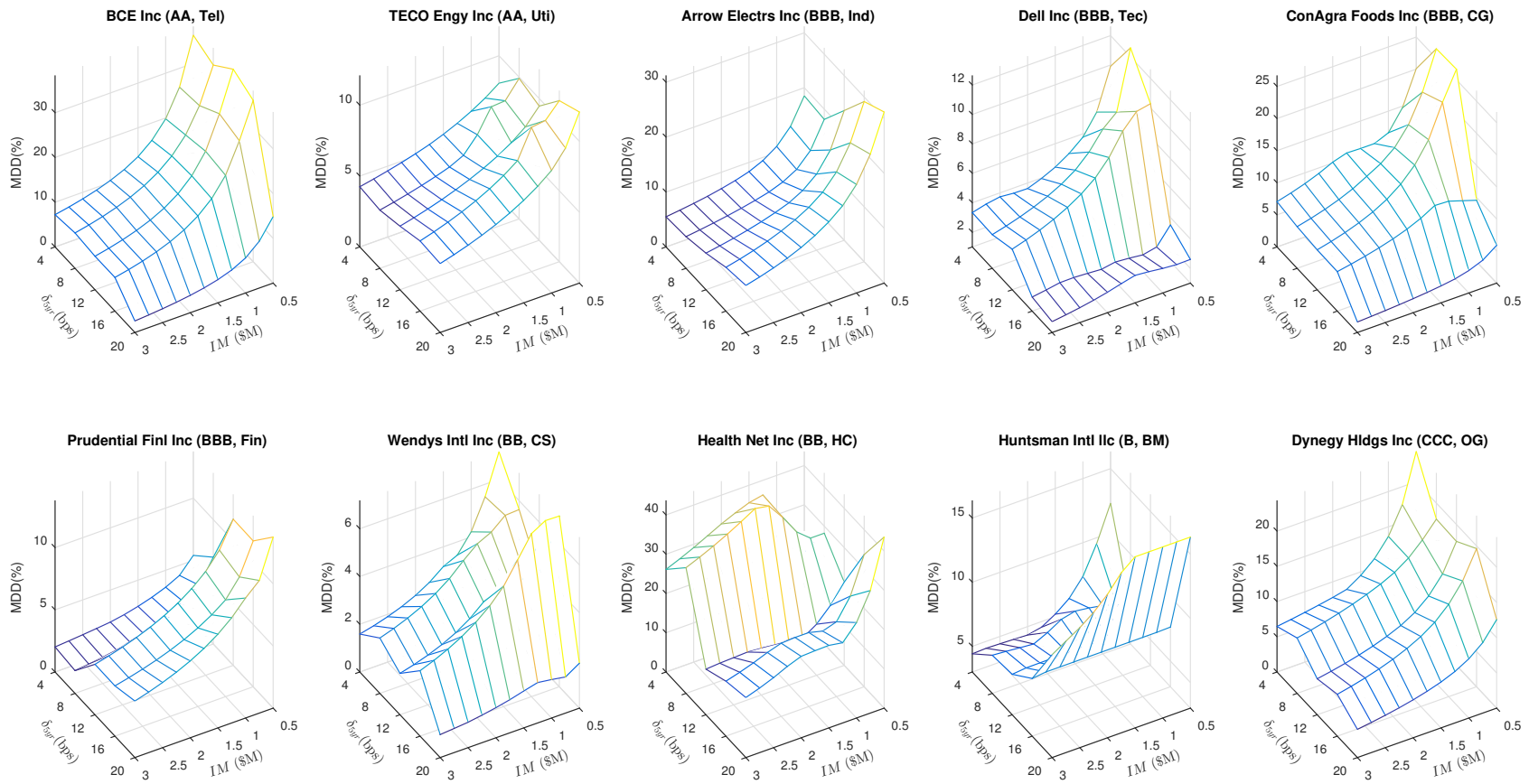
Figure 15: Time Series of In-sample Capital Growth in 10 Alternative Cases

This figure shows the in-sample capital growth, which is the cumulative capital gain over time, of the 10 firms in the second half of the sample (Jul2008 - Oct2011). Their names, ratings, and sectors are shown in the subtitles. The initial capital deposit is $\$ 1 \mathrm{M}$.
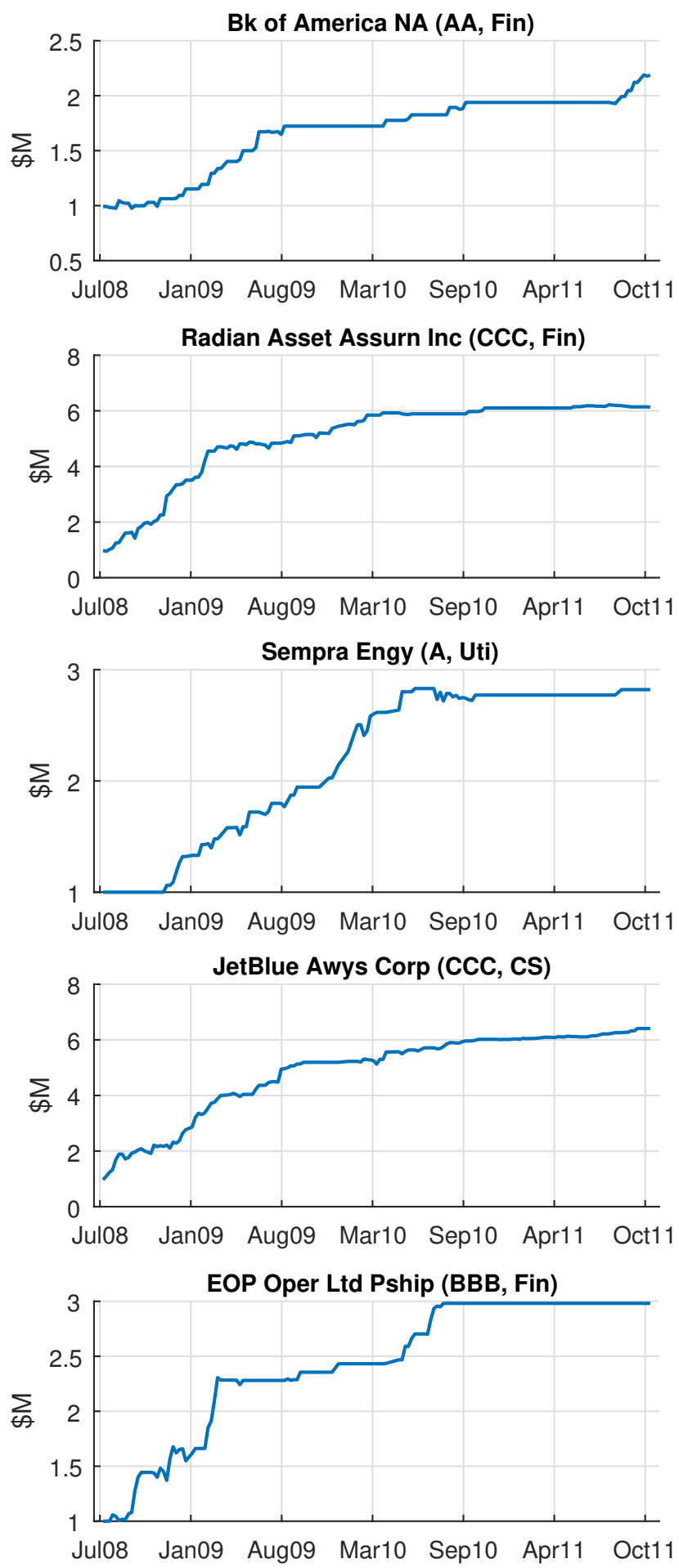
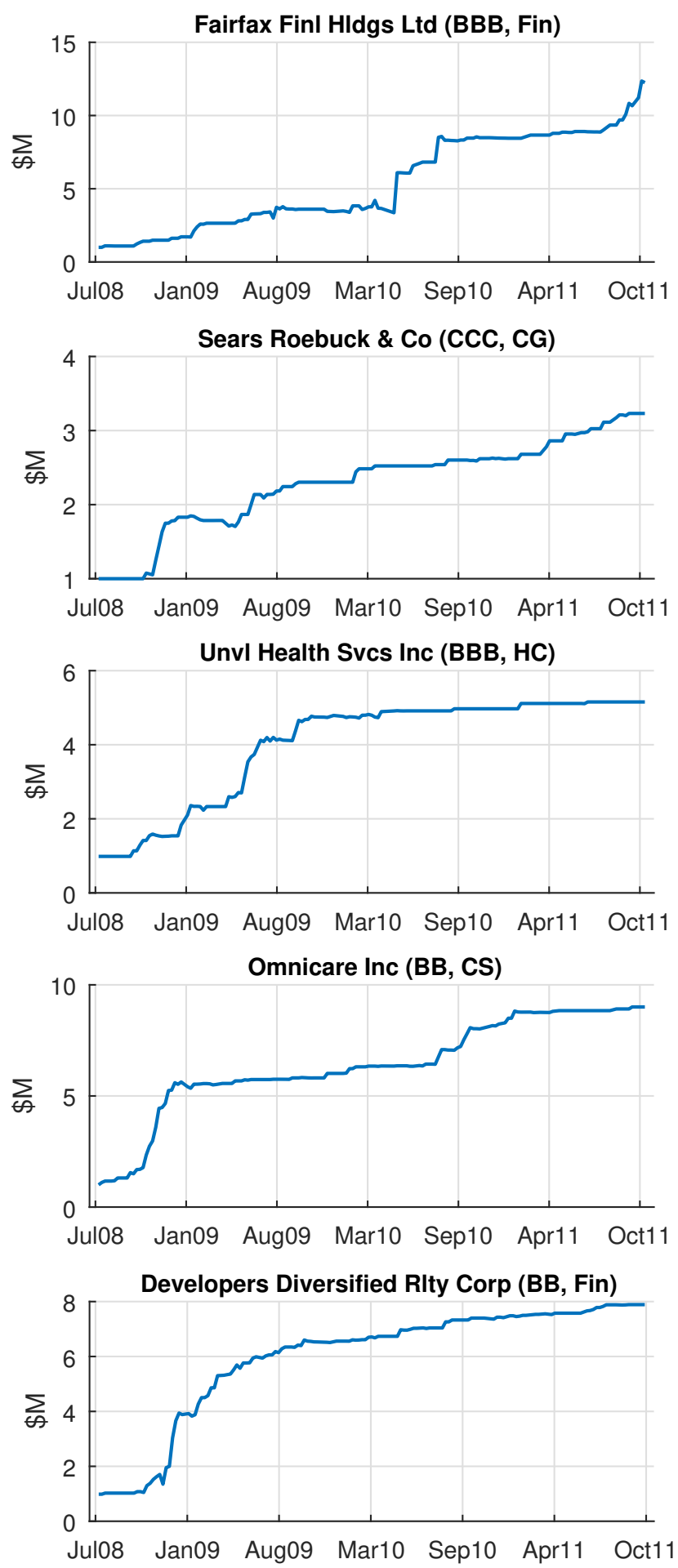
Figure 16: Time Series of Out-of-sample Capital Growth in 10 Alternative Cases

This figure shows the out-of-sample capital growth, which is the cumulative capital gain over time, of the 10 firms in the second half of the sample (Jul2008 - Oct2011). Their names, ratings, and sectors are shown in the subtitles. The initial capital deposit is $\$ 1 \mathrm{M}$.
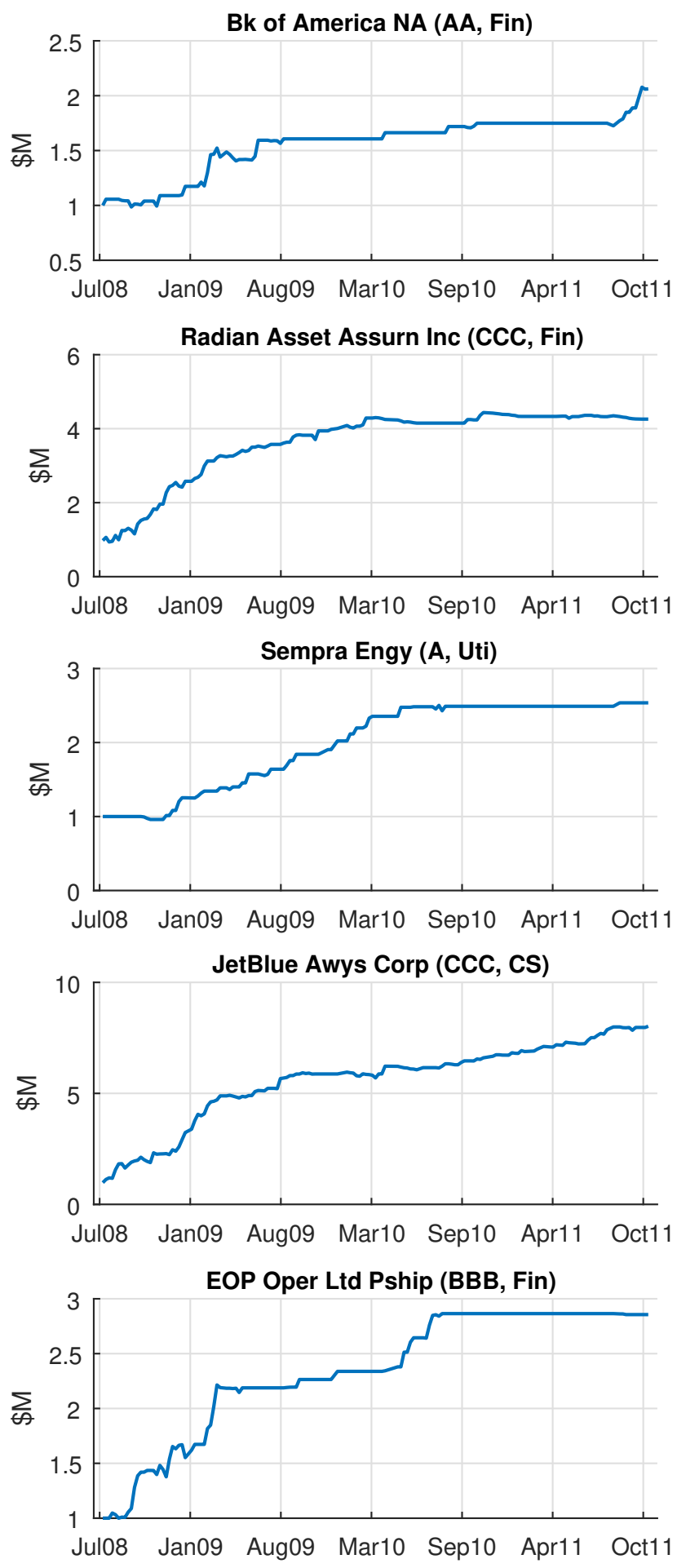
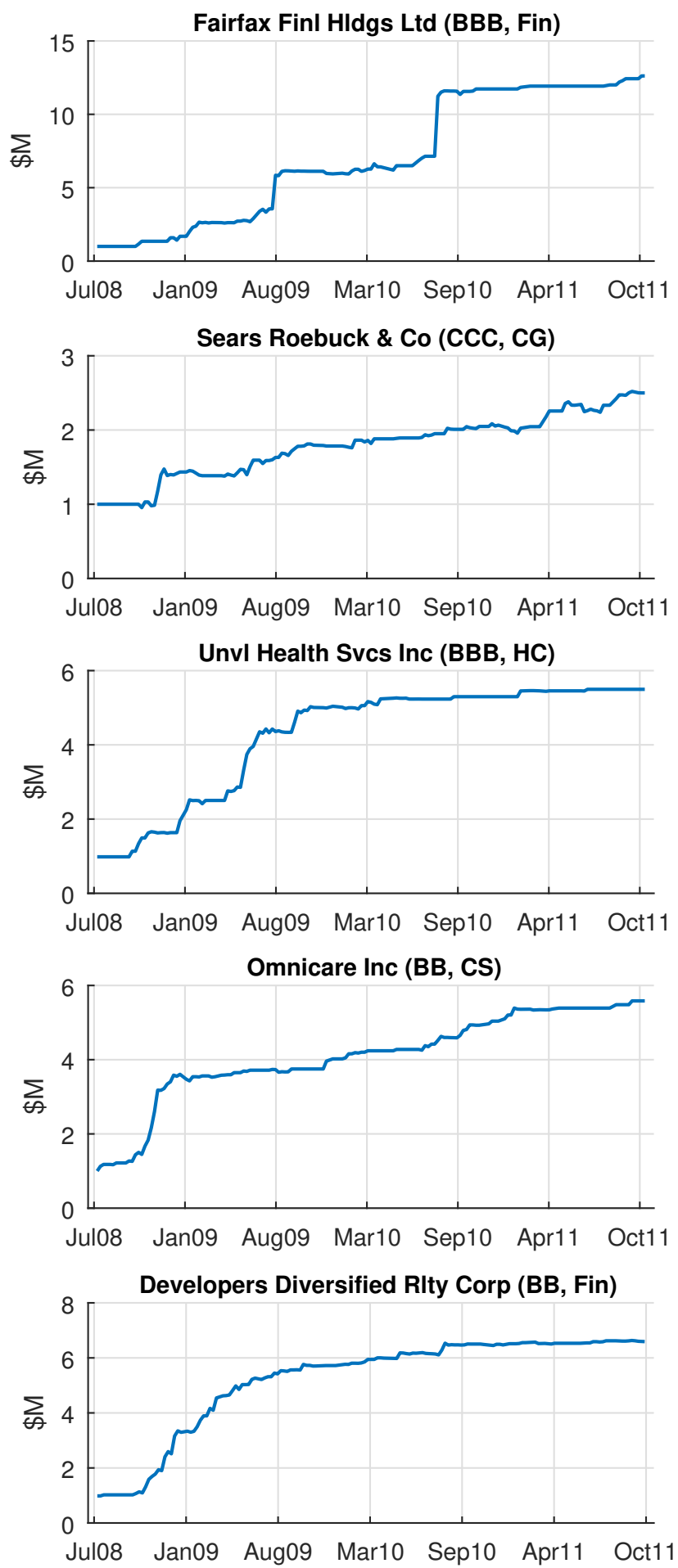
Figure 17: Time Series of In-sample Capital Gain and Loss in 10 Alternative Cases

This figure shows the in-sample capital gain and loss of the 10 firms in the second half of the sample (Jul2008 - Oct2011). Their names, ratings, and sectors are shown in the subtitles.
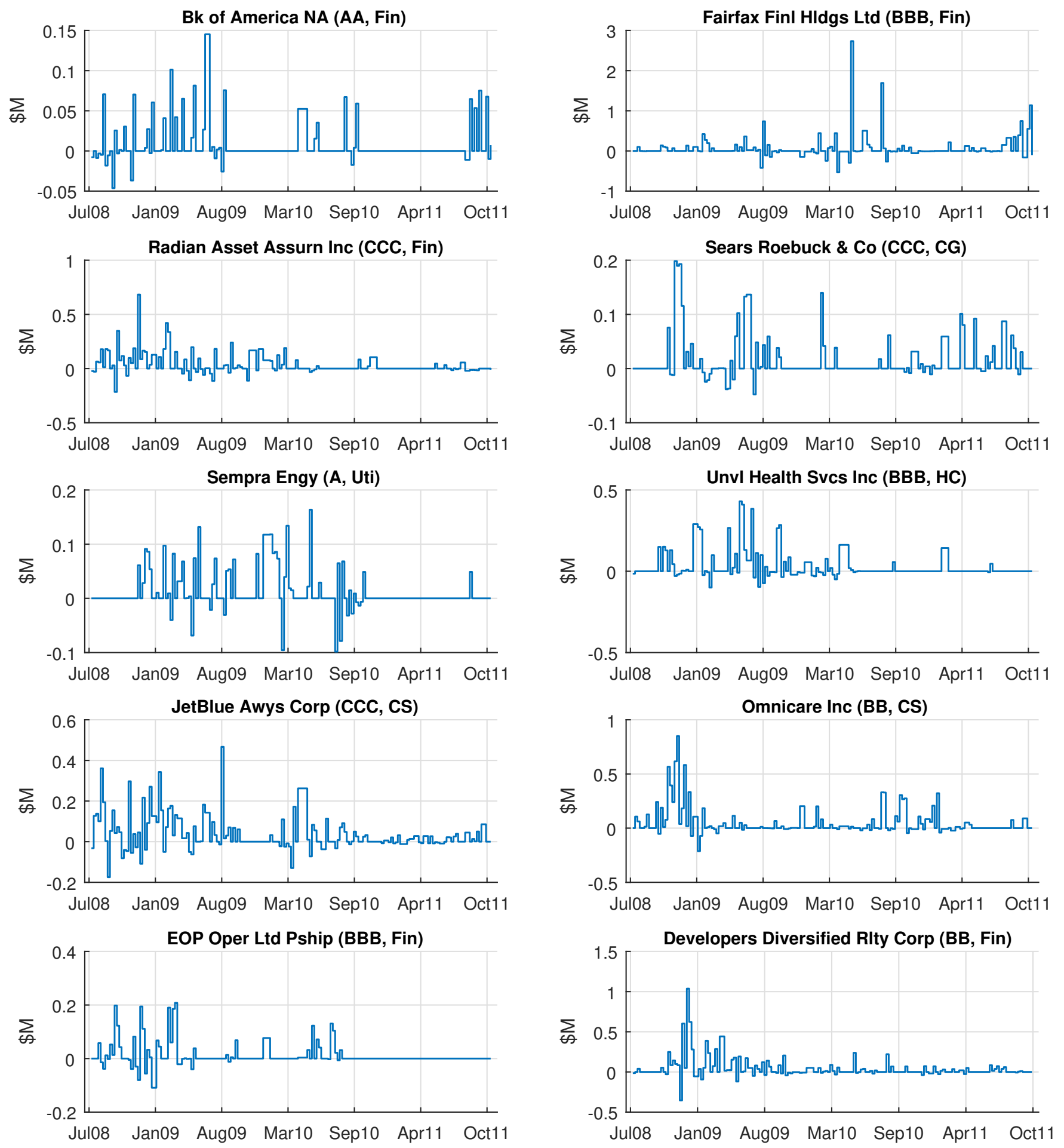
Figure 18: Time Series of Out-of-sample Capital Gain and Loss in 10 Alternative Cases

This figure shows the out-of-sample capital gain and loss of the 10 firms in the second half of the sample (Jul2008 - Oct2011). Their names, ratings, and sectors are shown in the subtitles.
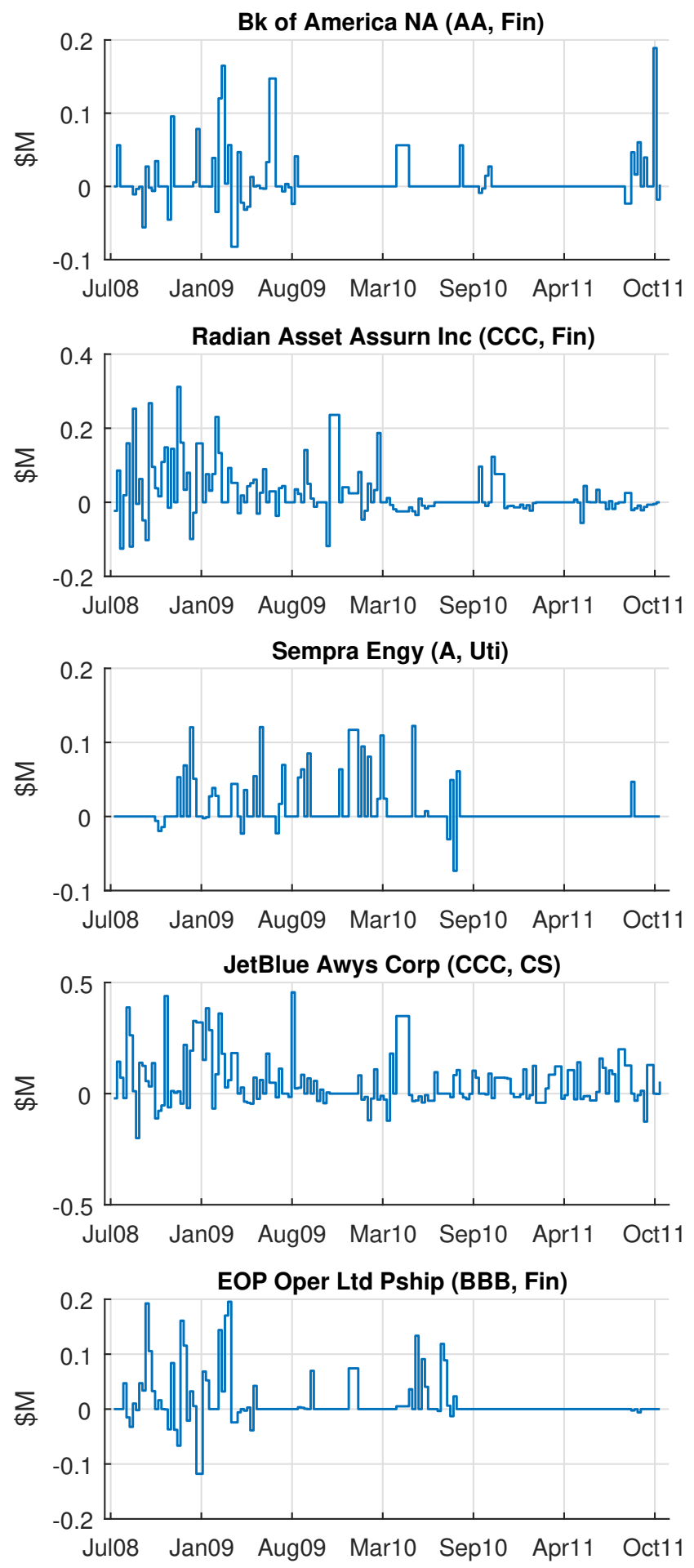
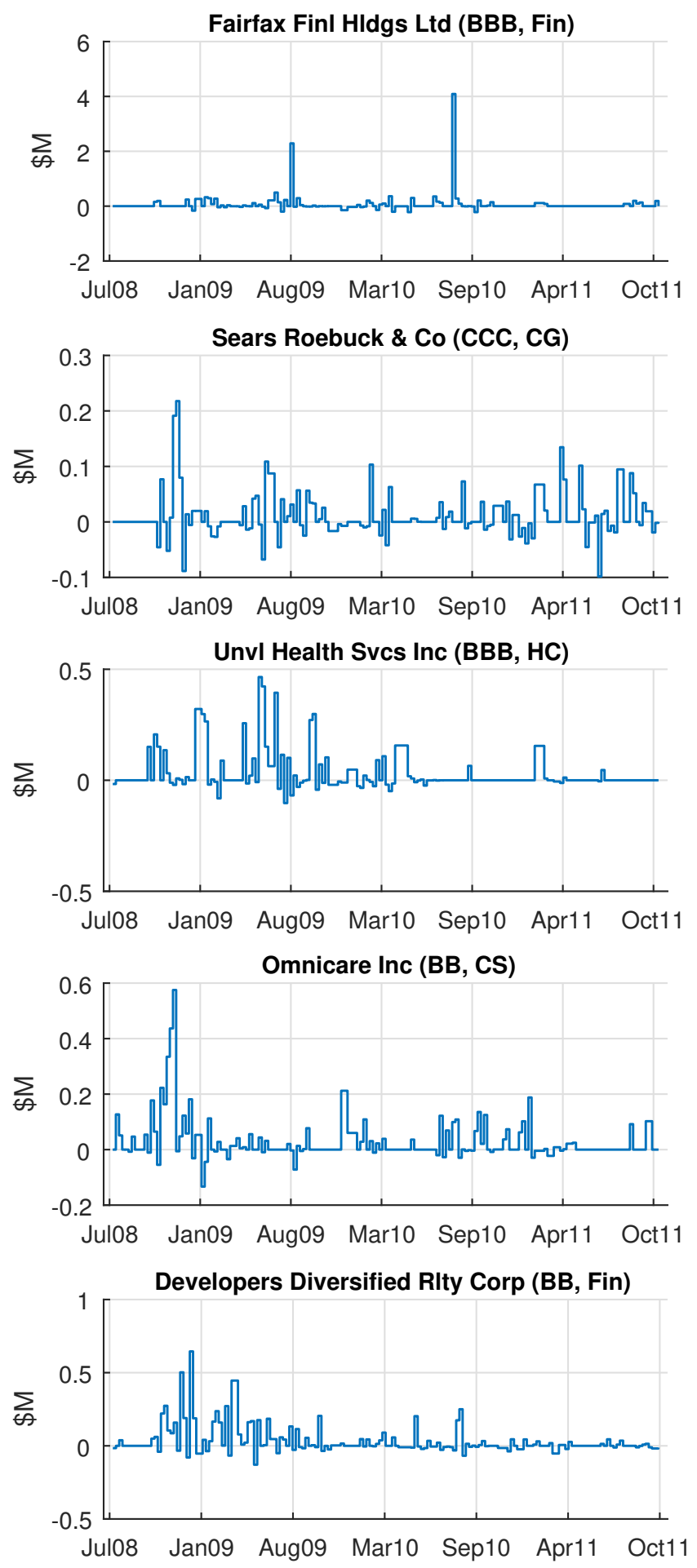
Figure 19: Histograms of In-sample Weekly Returns in 10 Alternative Cases

This figure shows the histograms of the in-sample weekly raw returns of the 10 firms in the second half of the sample (Jul2008 - Oct2011). Their names, ratings, and sectors are shown in the subtitles.
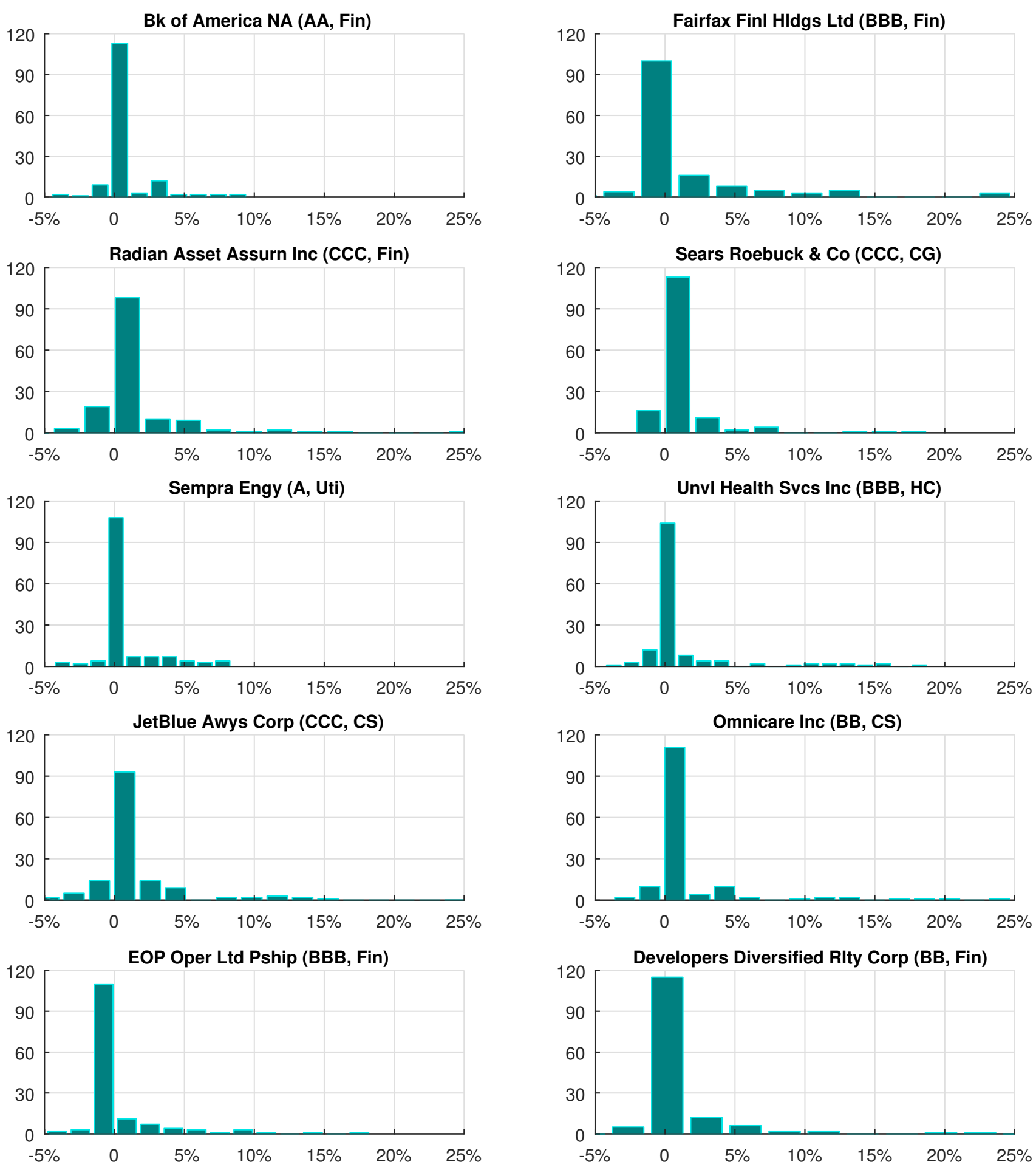
Figure 20: Histograms of Out-of-sample Weekly Returns in 10 Alternative Cases

This figure shows the histograms of the out-of-sample weekly raw returns of the 10 firms in the second half of the sample (Jul2008 - Oct2011). Their names, ratings, and sectors are shown in the subtitles.
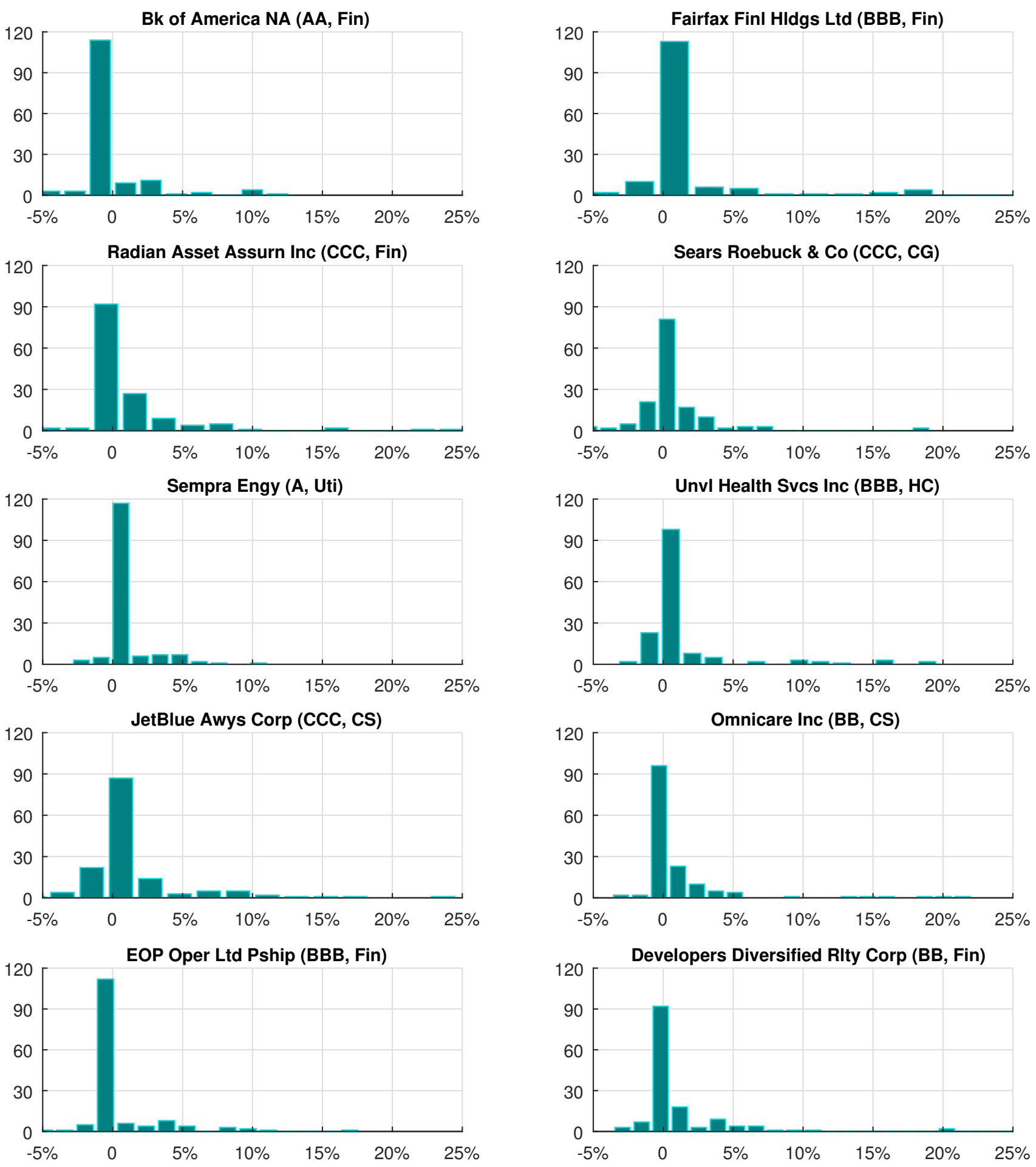


\section{References}

Ang, A. and Longstaff, F. A. (2013). Systemic sovereign credit risk: Lessons from the US and Europe. Journal of Monetary Economics, 60(5):493-510.

Arakelyan, A. and Serrano, P. (2012). Liquidity in credit default swap markets. Working paper, Universidad Carlos III de Madrid.

Biswas, G., Nikolova, S., and Stahel, C. W. (2014). The transaction costs of trading corporate credit. Available at SSRN 2532805.

Diebold, F. X. and Li, C. (2006). Forecasting the term structure of government bond yields. Journal of econometrics, 130(2):337-364.

Duffee, G. R. and Stanton, R. H. (2012). Estimation of dynamic term structure models. The Quarterly Journal of Finance, 2(02).

Heller, D. and Vause, N. (2012). Collateral requirements for mandatory central clearing of over-thecounter derivatives. Technical report, Bank for International Settlements.

Nelson, C. and Siegel, A. F. (1987). Parsimonious modeling of yield curves. The Journal of Business, 60(4):473-89.

Pan, J. and Singleton, K. (2008). Default and recovery implicit in the term structure of sovereign CDS spreads. The Journal of Finance, 63(5):2345-2384.

Yu, J. (2012). Bias in the estimation of the mean reversion parameter in continuous time models. Journal of Econometrics, 169(1):114-122. 